\title{
ANISOTROPY \\ OF PRIMARY COSMIC RADIATION \\ AT THE EARTH
}

A Thesis

Submitted to

Faculty of Graduate Studies, University of Manitoba, in partial fulfillment of the requirements of the Degree of Doctor of Philosophy.

\author{
By \\ Robert P. Bukata \\ Winnipeg, Manitoba \\ August, 1964
}

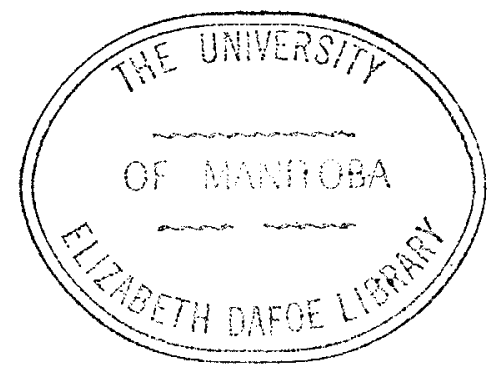


The design and construction details of a pair of geometrically identical, equatorially mounted, narrow angle, high energy particle telescopes are described. The techniques employed in utilizing these telescopes to effect an experimental study of energetic, directionally selected $\mu-m e s o n s$ at the University of Manitoba, Winnipeg are also described.

Experimental results obtained from March I, 1963 to July 23, 1964 are presented which indicate large anisotropies $(\sim 4-19 \%)$ of the primary cosmic radiation $>\sim 40 \mathrm{Bev}$ arriving at the earth's surface in or near the equatorial plane of the celestial sphere. Preferred arrival directions for this radiation appear to be defined by Right Ascention approximately $20.5 \mathrm{~h}, 10 \mathrm{~h}, 16 \mathrm{~h}$ and $4-6 \mathrm{~h}$. Evidence is presented which is suggestive of a solar modulation of the primary radiation as it interacts with the inner solar system.

Also included are calculations of intensity-zenith angle distributions determined for large zenith angle orientations $\geq 49.9^{\circ}$. 


\section{ACKNOWLEDGEUENTS}

Sincere gratitude is extended to the author's research director, Dr. S. Standil for his helpful guidance throughout the duration of the experimental work and the preparation of this thesis.

The help of Mr. R. H. Batten and his valuable counselling on the mechanical aspects of the telescope construction are gratefully acknowledged, as are the efforts of the Physics Department Technical Staff, with special thanks to Mr. R.C. Lamb.

Appreciation is extended to Mr. F. Konopasek, Mr. R.S. Foulds, and Mr. W. Bank for their help with the electronics.

The author would like to thank Dr. B.G. Whitmore, Dr。 H॰R. Coish, Dr. D.H. Hall, Dr, S.K. Sen, Dr॰ B.G. Hogg, Mr. F.K. Chin, and Mr. R.J. Lockhart for their interest in the experiment and their valuable suggestions.

Thanks are due to Dr. M. Bercovitch of the Chalk River Nuclear Laboratories, through whose courtesy asymptotic viewing direction calculations were provided to this laboratory。

The daily report on atmospheric conditions received from the Department of Transport, Winnipeg, is acknowledged with thanks.

Financial assistance from the National Research Council of Canada has made this research possible. In particular, the author is personally grateful for the N.R.C. scholarship awards granted him while this work was being undertaken.

Finally, special thanks are directed to the author's sister, Mrs. JoL. Scott, for graciously accepting all the manuscripts for typing。 
TABLE OF CONTENTS

Page

Abstract

Acknowledgements

Table of Contents

1. PART ONE: GENERAL INTRODUCTION I

1.I The Nature of the Primary Radiation 2

1.2 The Nature of the Secondary Radiation 4

1.3 Intensity Variations

$\begin{array}{ll}\text { 1.4. The Earth's Magnetic Field } & 14 \\ \text { Asymptotic Cones of Acceptance (Sky Windows) } & 15\end{array}$

Magnetic Cut-off Rigidities 18

2. PART TWO: NATURE OF THE CURRENT RESEARCH

3. PART THREE: DESIGN AND PERFORTANCE OF THE EQUIPMENT 36

3.I The Construction of the Cosmic Ray Telescope 37

3.2 The Telescope Geometry 49

3.3 The Scintillation Detectors 51

3.4 The Electronic System 55

3.5 Initial Electronic Alignment and Calibration 63

3.6 Experimentation 69

4. PART FOUR: DISCUSSION OF OBSERVATIONS 72

4.I Experimental Results 73

4.2 Conclusions 122

5. PART FIVE: APPENDICES 124

Appendix I: Estimate of the Minimum Proton Energy 125

Appendix II: Calculation of Zenith and Azimuth 129

Appendix III: The Alignment of the Polar Axis 138

References $\quad 142$ 
$\underline{P A R T} \quad \underline{O N E}$

GENERAL INTRODUCTION 
1.1 THE NATURE OF THE PRIIARY RADIATION

The advances made in the last fifteen years on large stratospheric balloons, radio chemistry, nuclear emulsions and radio telescopes, coupled with the progress made in low level beta and gamma counting techniques have linked cosmic ray physics as an integral part of many related researches. These researches include such fields as astrophysics, geomagnetism, solar physics, meteorology, oceanography, particle physics and biophysics.

The continuous stream of high energy primary cosmic particles has been reported by Waddington (1960) to be about $86 \%$ protons and $12.5 \%$ helium nuclei with heavier nuclei (up to $Z=26$ ) constituting about $1-1.5 \%$ of the total flux. Galactic cosmic radiation at the top of the terrestrial atmosphere is some 0.3 protons $\mathrm{cm}^{-2} \mathrm{sec}^{-1}$ sterad $^{-1}$ with a mean particle energy of about 2 Bev giving a total energy density of some $5.0 \times 10^{-13}$ erg $\mathrm{cm}^{-3}$.

The integral energy spectrum of the primary radiation is written for total energies $B$ of the incoming proton in the form

$$
N(>E)=\int_{B}^{\infty} n(E) d E
$$

where $N(>E)$ is the number of cosmic rays per unit solid angle, square centimeter and second with total energies at least $\mathbb{E}$ Bev,

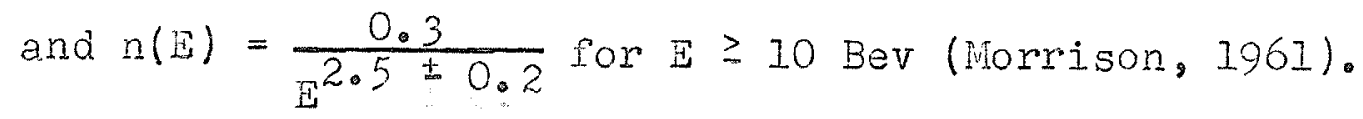


This integral spectrum is generally obtained from direct measurement and Clark et al (1957) review some of the experimental results obtained by various investigators. The primary energies $\mathrm{I}$ range from a few hundred $\mathrm{Mev}$ to at least $10^{20} \mathrm{ev}$.

The unique stream of these high energy radiations has led to much speculation as to its origin, and although there is no one definite theory that can deal completely with all the com plex phenomena associated with the cosmic radiation, there are two speculative theories that are currently very popular. The first theory propounds the view that the cosmic particles have been present since Creation, were created at their maximum energies and have come to lose some of that energy in their galactic wanderings. The second theory proposes that these particles were and are being created at minimal energies from certain localized sources within and/or without the galactic disk and are accelerated to their extremely high energies by some acceleration mechanism such as that suggested first by Fermi (1949) and later modified by Anderson (1960). This mechanism is one in which the cosmic radiation receives its energy by collisions with the magnetic fields produced by the motion of intergalactic clouds of ionized hydrogen. Further details and criticisms of the acceleration mechanisms are considered by Morrison (1961). The possible sources of these particles include supernovae and radio nebulae. Alfven (1958) 
has presented evidence against the sun's being a primary source of cosmic radiation, although it certainly does contribute to the low energy cosmic flux below $10^{7} \mathrm{ev}$. Simpson (1958), Katzman (1959) and others have reported that the energy of emitted solar radiation is a function of the intensity of the solar activity, and that during the active period of the solar cycle the sun can contribute very significantly to the intermediate energy cosmic spectrum above $10^{7} \mathrm{ev}$. Any consistent model of cosmic ray origin must logically consider the problems of particle injection, acceleration, stirring, storage, energy losses and possible high energy cut-offs in the allowed energy spectrum.

In addition to the article by Morrison (1961), there are several excellent reviews on the primary radiation such as those presented by Peters (1952, 1959) who surveyed cosmic ray physics from 1947-1959, Alfven (1949), Richtmeyer and Teller (1949) who discuss the origin of cosmic radiation, and Singer (1958a) who has considered the importance of the charge spectrum of the cosmic primaries for $Z>20^{+}$

\subsection{THE NATURE OF THE SECONDARY RADIATION}

The primary radiation upon entering the upper portion of the earth's atmosphere undergoes collisions with the heavier atmospheric nuclei. These collisions produce particles which give rise to further nuclear and electromagnetic interactions

${ }^{+}$See also Ginzburg, V.L. (1962), Uspekhi, 4, 4, 553. 
resulting in the creation of the secondary radiation. These secondaries consist of nucleons and a wide variety of elementary particles. The nucleons take part in further inelastic collisions giving rise to a nucleonic cascade while the various elementary particles very quickly give rise to charged and neutral $\pi$-mesons. The uncharged $\pi$-mesons decay into $\gamma$-rays which then initiate electron-photon cascades via bremsstrahlung and pair production. The charged pions (mean life time of $2 \times 10^{-8} \mathrm{sec}$ ) either participate in further nuclear interactions or decay into $\mu$-mesons. Because of their longer life-time $\left(2 \times 10^{-6} \mathrm{sec}\right)$ many of the relativistic $\mu$-mesons succeed in reaching the surface of the earth.

Auger (1936), by the observation of the absorption of the sea-level radiation in lead pointed out the existence of a 'hard' and 'soft' component of the secondary radiation, the soft component being that portion of the radiation which could be absorbed by $10 \mathrm{~cm}$ o of lead. Rossi (1933) using a triple coincidence counting technique indicated the presence of showerproducing particles which were later identified with the soft component. Since then (Rossi, 1952) the soft component has been associated with the electron and photon secondaries formed in the electromagnetic cascades while the hard component has been associated with the $\mu$-mesons (about 95\% of the hard component intensity at sea level) of Bev energies plus nucleons 
of the primary radiation.

The phenomenology of nucleon-nucleon interactions is discussed extensively by Gammel and Thaler (1960) and Sitte (1961). A detailed explanation of shower production, which will not be discussed here, would take into consideration the possibilities of ionization losses, angular spread at low energies, Compton scatterings and photoelectric effects. The problem of electron-photon cascade theory has been discussed in a review article by Rossi and Griesen (194I) as well as by Rossi (1952) and Sitte (1961). Griesen (1956) has also applied the cascade theory to the study of extensive air showers (EAS), a discussion of which may be found in the book by Galbraith (1958).

Puppi (1956) discusses the work of several investigators in their determination of the $\mu$-meson spectrum and presents differential and integral energy spectra at sea level. The generation of the $\mu$-spectrum from the parent $\pi$-mesons is also discussed theoretically and compared with the observations of such investigators as Rossi (1948), Owen and Wilson (1955), Puppi and Dallaporta (1952), Glaser et al (1950), George (1952), Kraushaar (1949) and Conversi (1950). The reference point taken in Puppi's article is the measured vertical intensity of muons below $15 \mathrm{~cm}$. of lead, at sea level and at $50^{\circ} \mathrm{N}$ recorded by Rossi (1948) and Puppi and Dallaporta (1952) to be

$$
I_{\mathrm{V}}=(0.83 \pm 0.01) \times 10^{-2} \mathrm{~cm}^{-2} \mathrm{sec}^{-1} \mathrm{sterad}^{-1} \text {. }
$$


The differential momentum spectra of muons at various zenith angles have been experimentally determined by the Melbourne group (Moroney and Parry, 1954) and discussed by Fowler and Wolfendale (1961)。

The directional intensities of cosmic radiation in the atmosphere (Ray, 1961) are dependent upon altitude, latitude, longitude, zenith angle, azimuth angle and time. The time variations remaining after corrections for atmospheric effects are due to changes in intensity of the primary radiation at the top of the atmosphere. The directional intensity $j$ is defined such that $j(\phi, \psi)$ dwdA represents the total number of particles passing through and perpendicular to an element of area $d A$ per second and coming within a solid angle d $\omega_{0}$ The zenith angle in the earth's reference frame is $\phi$ and the azimuth angle is $\psi$. The azimuthal variation is usually very small, if not completely negligible. However, it is customary to fit the zenith variation to a $\operatorname{Cos}^{n} \not \phi$ curve. The expression

$$
j=j(0) \cos ^{n} \phi
$$

is quite adequate for most practical cases. In this expression the azimuthal dependence is neglected and $j(0)$ represents the vertical intensity (i。e. the intensity for $\phi=0^{\circ}$ ). The value of $\mathrm{n}$ for the total $\mu$-meson intensity is generally found to decrease continuously from a value of about 2 at sea level to $O$ at the top of the atmosphere. However, recent experi- 
ments (Moroney and Parry, 1954; Roe and Ozaki, 1959) have shown that there are more very high energy $\mu$-mesons incident at high zenith angles than from the vertical. These results are in agreement with the theoretical discussions of Budini and Moliere (1953) which show that $\mathrm{n}$ becomes negative for $\mu-$-meson energies near 100 Bev. Smith and Duller (1959) have explained these effects in terms of $\pi$-meson decay and absorption phenomena occuring in the upper atmosphere.

\subsection{INTENSITY VARIATIONS}

The existence of several types of cosmic ray time variations is now well established. Elliot (1952), Simpson et al (1952), Cocconi (1961) and Dorman (1963) give extensive discussions of the work done on time variations of cosmic ray intensity along with discussions of the prominent types of variations. The non-periodic variations are produced by fluctuations in atmospheric temperature, barometric pressure, height of the atmospheric meson-production level, and occasional large scale effects of magnetic storms and solar flares. The four known periodic variations have periods of 12 solar hours, 24 solar hours, 27 days, and 1 year. An 11 year variation also exists which is associated with the 11 year solar cycle. Forbush (1954), Fenton et al (1958) and others have shown cosmic ray intensity to anticorrelate with solar activity. 
Myssowsky and Tuwim (1928) first observed the negative correlation between $\mu$-meson intensity and barometric pressure, which is to be expected from atmospheric absorption considerations. Blackett (1938) explained the negative temperatureintensity correlation coefficient on the basis of the instability of the $\mu$-meson. Hess (1942) reported and Hogg (1947) confirmed that the temperature coefficient suffers a seasonal variation, being twice as great in winter as in summer.

Duperier (1944) assumed the daily variations of $\mu$-meson intensity to be described by the regression equation

$$
\delta I=A_{1} \delta B+A_{2} \delta H+A_{3} \delta T
$$

where $I$ is the deviation from the mean intensity, $\delta B$ is the deviation from the mean barometric pressure, $\delta H$ is the corresponding deviation from the mean height of the meson-production level, $\delta \mathrm{T}$ is the deviation from the mean of the temperature of the atmospheric layer between the $200 \mathrm{mb}$ and $100 \mathrm{mb}$ layers, $\mathrm{A}_{1}$ is the absorption coefficient, $\mathrm{A}_{2}$ is the decay coefficient (probability of meson decay per unit path length) and $A_{3}$ is the temperature coefficient.

Duperier's theory, however, has met with difficulties when applied to experimental results. Barret et al (1952) removed some of the difficulties by indicating that often correlation exists between $A_{1}, A_{2}$, and $A_{3}$, and that the three 
coefficients cannot be treated independently.

Various methods for obtaining barometric pressure coefficients for the ground level variations have been described by Janossy and Rochester (1944), Trefall (1955), Wada (1960), Fenton et al (1961) and Lapointe and Rose (1962). The major work on atmospheric variations has been concerned with the meson and nucleon components of the secondary radiation. Preliminary results on the photon component have been presented by Bukata (1960), Bukata et al (1962) and Chin et al (1962).

Several theories have been put forward to explain the cosmic ray variations that persist after atmospheric effects have been eliminated. In recent years attention has been directed towards physical processes that might take place within the interplanetary medium which would affect the intensity at the earth's surface. The variation theories are generally classified into two categories, those in which the incoming particles undergo a change in energy, and those in which existing interplanetary magnetic fields determine the trajectories of the charged primaries.

Energy modulation theories have been proposed by Nagashima (1951) and Ehmert (1959) in which they attribute both Forbush decreases (see Forbush, 1954) in intensity and the 11 year variation to a sudden increase in potential of the earth relative to distant space. Alfven (1954) and Dorman (1957) 
suggested the cosmic ray variations associated with magnetic storms are due to the energy losses of cosmic particles as they encounter the solar corpuscular streams and interact with the magnetic fields frozen therein. Singer (1958b) suggested the existence of a predominent inverse Fermi mechanism as the cause of Forbush decreases. In such a mechanism particles will lose energy to the expanding cloud from the sun in a series of overtaking collisions.

Magnetic field modulation theories suggest that cosmic ray intensity is modified not by the particle's changing energy upon interacting with a magnetic plasma, but by the diffusion of these particles into the plasma cloud. According to Morrison (1956), such diffusion of cosmic radiation into the plasma cloud ejected during a large solar flare accounts for the creation of a barrier for particles outside the cloud, thereby producing a Forbush-type decrease.

Parker (1958), following up the earlier theory of Biermann (1957) assumed that the hydrodynamic flow of gas radially from the expanding solar corona stretches the lines of force of the solar dipole magnetic field outward into space in such a manner that the field in the inner solar system is nearly radial. The constant outflow of the 'solar wind' coupled with the solar rotation causes the 'radial' solar field to assume an Archimedes spiral ('garden-hose') configuration. Diffusion of cosmic radiation through such a garden-hose 
is then used to explain the Forbush decreases, Il year variations, etc. on the basis of solar activity. Elliot (1960), on the basis of a possible dipole field existing as a result of currents flowing in the solar corona and inner solar system, also offers explanations of the cosmic ray intensity variations. The diurnal variation of cosmic ray intensity produced by the solar wind is further discussed theoretically by Ahluwalia and Dessler (1962) and Dorman (1963). Other critical discussions of the various theories on cosmic ray variations may also be found in Webber (1962) and Parker (1963).

It has generally been considered that cosmic radiation approaching the earth is essentially isotropic. If cosmic ray anisotropy $\delta$ is defined as the ratio

$$
\delta=\frac{I \max -I \operatorname{Imin}}{I \max +\operatorname{Imin}} \times 100 \%
$$

between the fluxes in the directions of maximum and minimum intensities, no observations reliably yielding s greater than zero have been made for primary energies greater than $30-40$ Bev (Morrison, 1961). Most diurnal or annual variations which may be ascribed to primary anisotropies have thus far been attributed to energies below about $30 \mathrm{Bev}$. Jacklyn (1962) has presented underground results $(40 \mathrm{~m} \mathrm{woe.)} \mathrm{which} \mathrm{show} \mathrm{small}$ diurnal variations (of amplitude $\sim 0.10 \%$ ) in the vertical 
intensity averaged for the years 1958,1959 and 1960.

Even if large anisotropies exist far from the earth, the apparent isotropy of the primary radiation at the earth implies that the original trajectories of the individual cosmic particles have been completely randomized by the galactic magnetic fields. Anisotropies of the primary cosmic radiation (i.e. a preferred arrival direction at the earth's surface) might be expected at ultra high particle energies due to the decreasing effectiveness of the galactic fields in randomizing the arrival directions at the surface of the earth. Various investigators have therefore considered this possibility through a study of arrival directions of the ultra-high energy component $\left(\sim>10^{16} \mathrm{ev}\right)$. This type of investigation is generally carried out by a study of the extensive air showers using such detector arrays as described by the M. I. T. group (Clark et aI, 1961) and the Japanese group (Hasegawa et al, 1962). As a result of such studies there are indications of possible anisotropies in the arrival directions of the ultra-high energy radiation. For example, Linsley et al (1962) reported apparent anisotropies, $\delta$, of about $(25 \pm 7) \%$ for primaries $\sim 5 \times 10^{17} \mathrm{ev}$ and about $(70 \pm 30) \%$ for primaries $\sim 4 \times 10^{18} \mathrm{ev}$, with minima located in sidereal time between 12 hours and 18 hours, and between 18 hours and 24 hours, respectively. Hasegawa et al (1962) reported maxima in the region $\mathrm{R}_{\diamond} \mathrm{A}_{0}=$ 3 - 15 hours and minima in the region 15 - 18 hours for 
particles $10^{16}$ - $10^{18} \mathrm{ev}$. They interpreted their data as indicative of an anisotropy in a direction perpendicular to the Orion arm of the galaxy. Sakakibara and Sekido (1964) using harmonic analysis, however, have interpreted the data of Hasegawa et al as indicative of an anisotropy parallel to the galactic arm with maximum amplitudes of about $62 \%$ and $40 \%$ occuring from $R_{0} A_{0}=8 \pm 1 \mathrm{~h}$ and $2 \mathrm{I} \pm$ ih respectively.

Such anisotropies would certainly add substance to the localized source theory of the cosmic radiation.

\subsection{THE EARTH'S MAGNETIC FIELD}

The earth's approximately dipole field is of prime concern to any experimenter who effects a study of the cosmic radiation in the vicinity of the earth. The geomagnetic field's importance manifests itself in two aspects. First, due to the deflection of a charged particle in a magnetic field, the earth's field dictates a cut-off primary particle energy below which a particle cannot reach a particular location on the earth's surface. Also, particles possessing sufficient energies to reach the surface of the earth can only do so from a particular direction with respect to the detector location due to the constraints placed on the trajectory of the particle by the geomagnetic field.

Because of the importance of the influence of the earth's field on not only the investigation described in this thesis, but on all ground level investigations, these two aspects of 
the geomagnetic field will be discussed separately.

(a) ASYMPTOTIC CONES OF ACCEPTANCE (SKY UINDOWS)

A problem often confronting the cosmic ray physicist is the answer to the question 'If a particle leaves a point source far from the earth and travels essentially in a straight line unimpeded until it is influenced by the geomagnetic field, where will it strike the earth?" The method of analysis briefly described here concerned itself primarily with intensity increases associated with particles emitted during solar flares.

Trajectories of charged particles moving under the influence of a magnetic field have been integrated by Stormer (1931), Dwight (1950) and Schluter (1951). The general method of approach used was to consider an inverse problem. That is, rather than considering a positively charged particle moving towards the earth, a negatively charged particle was considered as being projected away from the earth. The integrated path of such a particle was then traced through the geomagnetic field to a distance sufficient that the earth's field was no longer effective in governing the particle's motion. Experimental models devised by Malmfors (1945), Brunberg (1953) and Brunberg and Dattner (1954) were used to attack the same problem from an experimental point of view. 
The unidirectional differential flux of cosmic radiation of charge $Z$ in the vicinity of the earth will generally be a function $F_{Z}(R, \underline{D}, t)$ of particle rigidity $R$, arrival direction D and time t. $F_{Z}(R, \underline{D}, t)$ is termed the source function. Firor (1954) showed that by assuming a simple source function for the cosmic flux, calculations for the deflections of a particle entering a centred dipole approximation to the earth's field would yield a certain number of regions on the earth's surface that are accessible to particles initially approaching the earth from some particular direction in space. (The direction Firor chose was from the sun). Firor termed these regions the impact zones for the particular cosmic ray source chosen. Recently McCracken (1962) extended the work of Firor as well as the concept of asymptotic cones of acceptance developed by Malmfors, Brunberg and others. The asymptotic direction of approach for a cosmic ray particle arriving at a specific point on the surface of the earth is the direction of the particle's velocity vector before entry into the earth's magnetic field. This direction can readily be related to a reference system fixed in the earth frame by translating the asymptotic velocity vector parallel to itself in space until it passes through the centre of the earth. The intersection points of such a line with the earth may then be defined in terms of either geographic or geomagnetic latitude $(\Lambda)$ and longitude $(\Psi)$. The values of 
$\Lambda$ and $\Psi$ are clearly functions of the primary particle energy $E$, the geographic latitude $\lambda$ and longitude $\beta$ of the station, zenith angle $\phi$, and the azimuth angle of observation $\psi$. For a detector such as a meson telescope which is equipped to observe a range of particle energies the re will be a set of such suitable asymptotic velocity vectors for a particular orientation of the telescope. Thus the detector will observe radiation from a spread of directions defined by the set of non-parallel asymptotic vectors. Such a set constitutes an asymptotic 'cone' of acceptable viewing directions for the particular detector. Hence, a detector in a particular orientation in the laboratory will sample radiation from an appropriate 'sky window'。

In his calculations of the asymptotic cones of acceptance for various detector locations. McCracken utilized the Finch and Leaton (1957) sixth degree approximation to the geomagnetic field and extended the integration (via computer programming) out to 25 earth radii.

Similar computations of asymptotic viewing directions were described and recorded for a variety of locations by Hatton and Carswell (1963), and through the courtesy of Dr. M. Bercovitch of the Chalk River Nuclear Laboratories calculations of asymptotic viewing directions appropriate to the experiments described in this thesis were provided to this laboratory. These sky windows are depicted in figure 21 . 
(b) MAGNETIC CUT-OFF RIGIDITIES

The magnetic rigidity $R$ of a charged particle moving in a circular path of radius $\rho$ in a magnetic field of strength $H$ is defined by

$$
R=H p=\frac{p c}{Z e}
$$

where $\mathrm{p}$ is the particle momentum

and $\mathrm{Ze}$ is the particle charge.

The earth's magnetic field deflects charged particles and prevents them from reaching the atmosphere unless the particles possess a magnetic rigidity in excess of some minimum value $R_{m} \cdot R_{m}$, which is termed the vertical cut-off rigidity, is dependent on both the charge on the particle and the geomagnetic latitude of the observation station.

Stormer (1955) showed that if the earth's field were treated as being that of a simple dipole, $R_{m}$ in $B V$ could be calculated for vertical arrival at a location of geomagnetic latitude $\lambda_{\mathrm{c}}$ by

$$
R_{m}=\frac{3}{10^{7}} \cdot \frac{M}{4 R^{2}} \cos ^{4} \lambda_{c}
$$

where $M$ is the dipole magnetic moment and $R$ the radius of the earth in c.g.s. units. It was found, however, that the above formula did not yield values of $R_{m}$ consistent with experimental observations. Rothwell (1959) suggested that $M$ be replaced with an effective magnetic moment $M_{a}$ for a geographic location 
agreeing with observations at that station. $M_{a}$ could be deduced from the local dip angle $\delta a$ and the local horizontal intensity $\mathrm{H}_{\mathrm{a}}$ by the relation

$$
M_{a}=\frac{H_{a} R^{3}}{\operatorname{Cos}\left[\tan ^{-1} \frac{\left(\tan \delta_{a}\right)}{2}\right]}
$$

Quenby and Webber (1959) also. in an attempt to bring Stormer's equation into closer alignment with the measured values of local cut-off momenta devised an artificial dipole for the earth's magnetic field which could be modified at the poles and equator to obtain consistency with observations. This hypothetical dipole then required the introduction of an effective magnetic latitude $\bar{\lambda}$ which was related to $\lambda_{c}, H_{a}, \mathbb{M}$, $R$, and $\delta_{a}$ via

$$
\bar{\lambda}=\tan -1\left[\frac{\tan \lambda_{c}}{1+\frac{H_{a} R^{3}}{M \operatorname{Cos} \lambda_{c}}}+\frac{\tan \delta_{a}}{2\left[I+\frac{M \operatorname{Cos} \lambda_{c}}{H_{a} R^{3}}\right]}\right]
$$

Quenby and Webber thus arrived at the expressions

$$
R_{m}=\frac{3}{10^{7}} \cdot \frac{M}{4 R^{2}} \operatorname{Cos}^{4} \bar{\lambda} \text { for } \lambda_{c}>40^{\circ}
$$

and $R_{m}=\frac{3}{107} \cdot \frac{\mathrm{M}}{4 R^{2}}\left[1+0.6 \frac{\mathrm{H}_{2} R^{3}-M \operatorname{Cos} \lambda_{c}}{M \operatorname{Cos} \lambda_{c}}\right] \cos ^{4} \bar{\lambda}_{c}$

$$
\text { for } \lambda_{c}<40^{\circ}
$$


Applying the expression for $\lambda_{c}>40^{\circ}$ to the location of the current research, Winnipeg, Manitoba (geomagnetic latitude $59^{\circ} 46^{\circ}$, dip angle $77.4^{\circ}$ and local horizontal intensity approximately 0.131 gauss) and taking the values $M=8.06 \times 10^{25} \mathrm{ergs}$ per gauss and $R=6.37 \times 10^{8} \mathrm{~cm}, R_{m}$ was calculated as being $0.643 \mathrm{BV}$. By way of comparison, values of $R_{m}$ range from nearly zero at the poles to about $20 \mathrm{BV}$ at equatorial regions. Tabulated computations of magnetic cut-offs for an extensive number of cosmic ray locations may be found in the report by Cogger (1960)。 
PART TWO NATURE OF THE CURRENT RESEARCH 
2. NATURE OF THE CURRENT RESEARCH

Time variation studies by means of continuous monitoring of the ground level cosmic ray intensity have been performed by many workers ever since the $1930^{\circ} \mathrm{s}$. The importance of the time variation studies of the primary cosmic rays is emphasized by the close relation of the cosmic ray variations to geophysical phenomena and interplanetary dynamical processes. The interplanetary dynamical processes are intimately connected with solar-terrestrial relations, being responsible for many conspicuous phenomena at the earth's surface such as aurora, solar cosmic ray enhancements, geomagnetic storms, Forbush decreases, variations in the trapped radiation belts and other modulation effects. Direct knowledge of many aspects of these problems may in many cases be obtained from a study of possible cosmic ray anisotropies, $i_{0} e_{\text {e the search for }}$ preferred arrival directions in space of galactic cosmic particles.

The ideal method of studying the primary radiation is to detect these particles above the earth's atmosphere before they have an opportunity to interact with the atmospheric nucleons. Earth-bound investigators, however, must content themselves with an indirect investigation of the primary particles through the behaviour of daughter secondaries which are formed by the primaries through their nuclear and electro- 
magnetic interactions with atmospheric nuclei.

Early studies on the time variations of sea level radiation were carried on with the use of ionization chambers, but more recent investigations utilize more selective neutron monitors and meson telescopes. The neutron monitor (Simpson, 1957) responds to the nucleonic component of the secondary radiation being most sensitive to the lower energy portion of the primary cosmic ray spectrum under about $10 \mathrm{Bev}$. The meson telescope, on the other hand, responds to the $\mu$-meson component produced by the higher energy portion of the primary radiation above about $10 \mathrm{Bev}$. This difference in energy response between neutron monitors and meson telescopes results in the meson telescope being less sensitive to the solar produced cosmic rays of low energy and consequently observations made on the variations of the meson component near sea level are more certain in their detection of corresponding variations in the galactic cosmic radiation.

Many of the meson telescopes in existence have employed GM counters as their detector heads (Elliot, 1957), while with the recent development of the scintillation phosphor the current trend has been the use of large area scintillators as the detecting system. The phosphor detectors have many distinct advantages over other forms of detectors. They do not suffer from chemical deterioration, they have essentially an infinite lifetime, they can readily be obtained in any desired geometri- 
cal configuration, and they require less complex electronic circuitry. These advantages then lead to improvements in overall stability and considerable ease of maintenance. Several meson telescopes located both at sea level and underground have been constructed in the past few years. Typical are those described by Bercovitch (1962), Mathews (1962) and coxell et al (1963). In addition there is now also a world-wide network of strictly comparable neutron monitors (Simpson, 1956). Thus both the nucleon and meson components of the secondary radiation near sea level have been and are currently still being given considerable attention from a variety of observation stations.

The current research also concerned itself with the continuous monitoring of the $\mu$-meson component at the surface of the earth. The location of the investigation was Winnipeg, Manitoba (geographic longitude $97.2^{\circ} \mathrm{W}$, geographic latitude $49.9^{\circ} \mathrm{N}$ and altitude 236 meters) and the detecting system was located in the cosmic ray penthouse situated on the roof of the Allen Physics Laboratory on the University of Manitoba campus. Although the idea of effecting a continuous monitor on the cosmic ray flux is not a new one, to the best of this investigator's knowledge the experimental method used in this case was both different and original. The standard conventional technique involved in the time variation study of ground level radiation is the construction of energy selective 
counter telescopes or neutron detectors which occupy some fixed orientation in the laboratory frame of reference. These detectors are usually erected in the vertical direction (for maximum counting rates) and are kept fixed in position throughout the duration of the investigation. Also there are extensive air shower experiments such as those described by Rossi (1959) wherein many detectors are arranged over several square meters and even acres, the data from these giving the direction and size of the shower, and from the size of the shower the energy of the primary particle is obtained. Here, too, however, the detectors are kept fixed in relation to the laboratory frame of reference. Thus, as the earth daily rotates about its axis, this rotation effects a selection of galactic viewing directions for the detecting system. In this manner, therefore, such detectors sample radiation from continuously varying directions in space ( $i_{0} e_{0}$ in a galactic frame of reference).

Since for a particular particle energy the asymptotic cones of acceptance which define the 'sky window' for a particular counter telescope are functions only of the zenith and azimuth orientation of the telescope arrangement, as the earth rotates carrying the stationary telescope with it, the detector remains fixed with respect to its sky window. Therefore, as the earth rotates, the telescope's cone of acceptance rotates with it. Thus the window rotates through space 
and the detector always samples radiation entering through it, this radiation coming from continuously varying directions in galactic space. This means that a counter telescope rigidly attached to the earth studies radiation from a particular window which constantly scans a region of the galaxy because of the earth's rotation.

The current investigation also utilizes counter telescope arrangements to experimentally study energetic directionally selected $\mu$-mesons, but with one distinguishing feature. The telescopes are not rigidly fixed in a particular orientation with respect to the surface of the earth, but rather are constrained to rotate in a particular manner. It is this property which enables a continuous monitoring of a fixed direction in the galaxy。

Consider the celestial sphere depicted in figure 1. The celestial sphere is the conventional representation of the sky as a spherical shell upon which the 'fixed' stars appear projected. Due to the ultra large distances involved, this shell displays the properties of a sphere of infinite radius, its centre generally taken to be at the location of the observer. Regardless of their separation, parallel lines converge to the same point on the celestial sphere.

As a consequence of the earth's rotation on its axis from west to east there is an apparent daily rotation of the celestial sphere around the earth from east to west. This 
FIGURE 1

The Celestial Sphere 


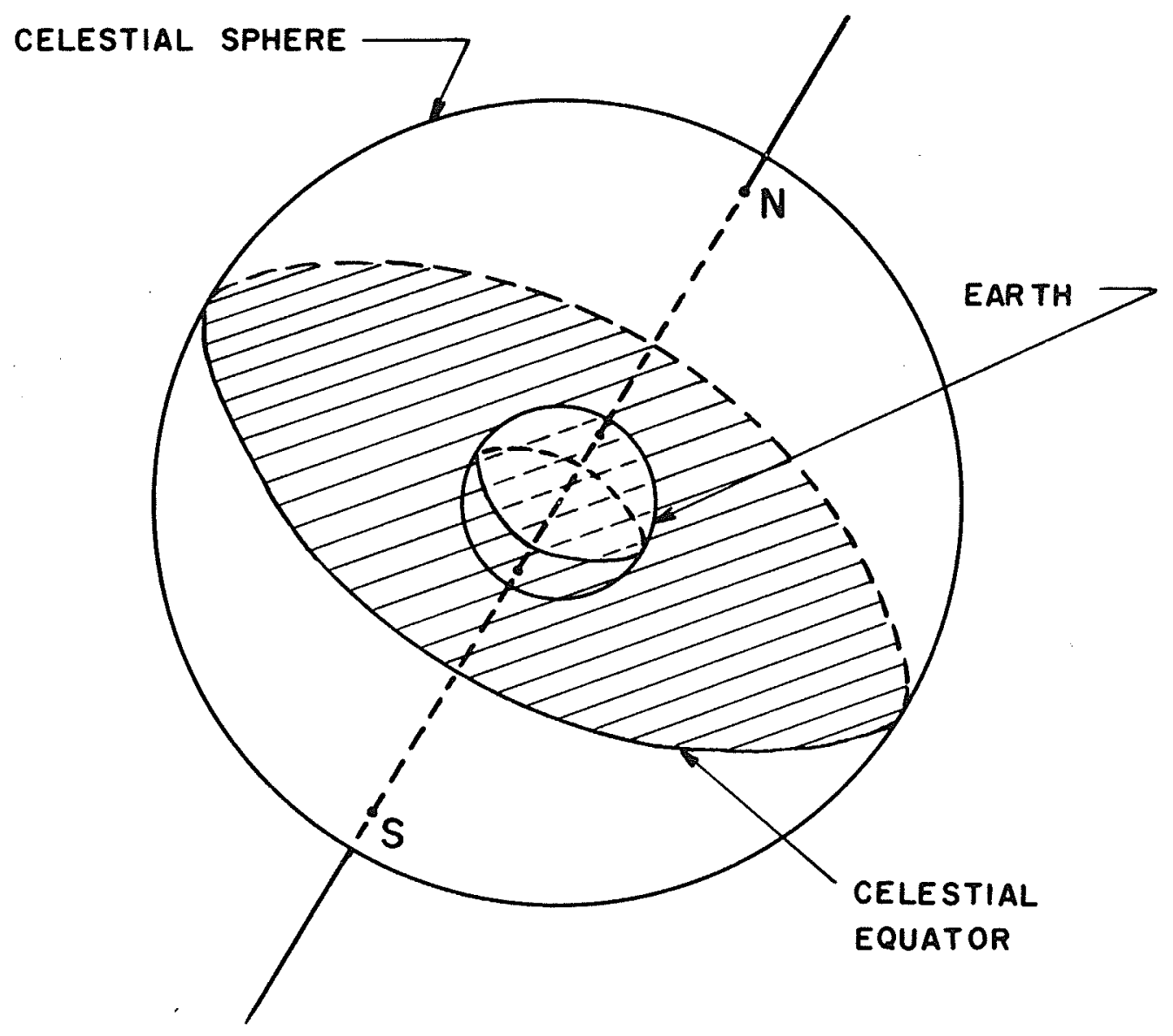


apparent daily rotation is termed the diurnal motion of the celestial sphere and the day involved is a sidereal day. The two points on the celestial sphere (labelled $\mathrm{N}$ and $\mathrm{S}$ in figure I) possessing no diurnal motion are termed the north and sourth celestial poles and are the intersection points with the projection of the earth's axis. The celestial equator is the great circle of the celestial sphere midway between the two celestial poles.

Thus, if an axis were taken parallel to the earth's axis and a telescope fastened at right angles to this axis, in physical space a straight line drawn along the major axis of the telescope would intersect at two points on the celestial equator separated by $180^{\circ}$. Further, if this axis were then caused to rotate in a direction east to west at the rate of 1 revolution per sidereal day, this would compensate for the apparent rotation of the celestial sphere, and each of the extremities of the telescope would then be confined to a continuous observation of the same two points on the celestial equator. In a similar manner, if a second telescope were added at right angles to both the axis of rotation and the first telescope, the two telescopes would always be trained along two fixed diameters of the celestial sphere. If each telescope were bi-directional, each diameter would then define two fixed directions in space. Each of the four directions thus defined would be $90^{\circ}$ away from the next. 
Due to the fact that the earth undergoes an annual revolution about the sun, the sun appears to be displaced in the heavens about two solar diameters per day and completely around the heavens in a year. Not only does the sun move eastward with respect to the stars, but it also undergoes a north-south movement during the year so that its path does not coincide with the celestial equator. The ecliptic is the sun's apparent annual path on the celestial sphere. The plane of the ecliptic is inclined about $23.5^{\circ}$ to the plane of the celestial equator.

If local time is reckoned in terms of a solar day, then due to the relative earth-sun motion, the solar day (defined as the time interval between successive appearances of the sun directly over the earth's prime meridian) is longer than the sidereal day (defined similarly for the earth-celestial sphere motion) by about three minutes and 55.9 seconds. Thus, if the axis upon which the telescopes are mounted were to be rotated at the rate of one revolution per solar day, the telescopes would not be constantly trained at four points on the celestial equator, but rather each point would shift $360^{\circ} / 365.25$ or a little less than $1^{\circ}$ eastward per solar day. Hence, during a solar year, each telescope would gradually trace out the entire celestial equator. Whereas a sidereal driven axis would use fixed diameters on the celestial equator as an experimental parameter, a solar 
driven axis would employ, understandably, a parameter which is related to the sun. This parameter is the projection of the earth-sun line in the plane of the celestial equator, and will be referred to as the PES line in all subsequent sections of this thesis. That is, at all times during such an experimental run the axis of each telescope would lie in the plane of the celestial equator and would remain in a constant orientation with respect to the PES Iine. Equivalently, a solar drive results in the daily motion of the viewing directions of each telescope eastward along the celestial equator at the same velocity as the component of the sun's velocity along the celestial equator in its apparent yearly motion along the ecliptic. For convenient reference, an SCI constellation chart showing the ecliptic and celestial equator is included and labelled as figure 2. This chart illustrates the celestial sphere expressed in Mercator projection.

In the design stages of this experiment the decision to utilize a solar drive was reached. A sidereal drive has the advantage of the ability to constantly observe intensity variations from a fixed point on the celestial equator while at the same time it suffers a disadvantage in that only a limited number of directions (four) can be observed during any one long-term run. Small anisotropies might well be undetectable using narrow angle telescopes if the choice of 


\section{FIGURE 2}

The SCI Constellation Chart 


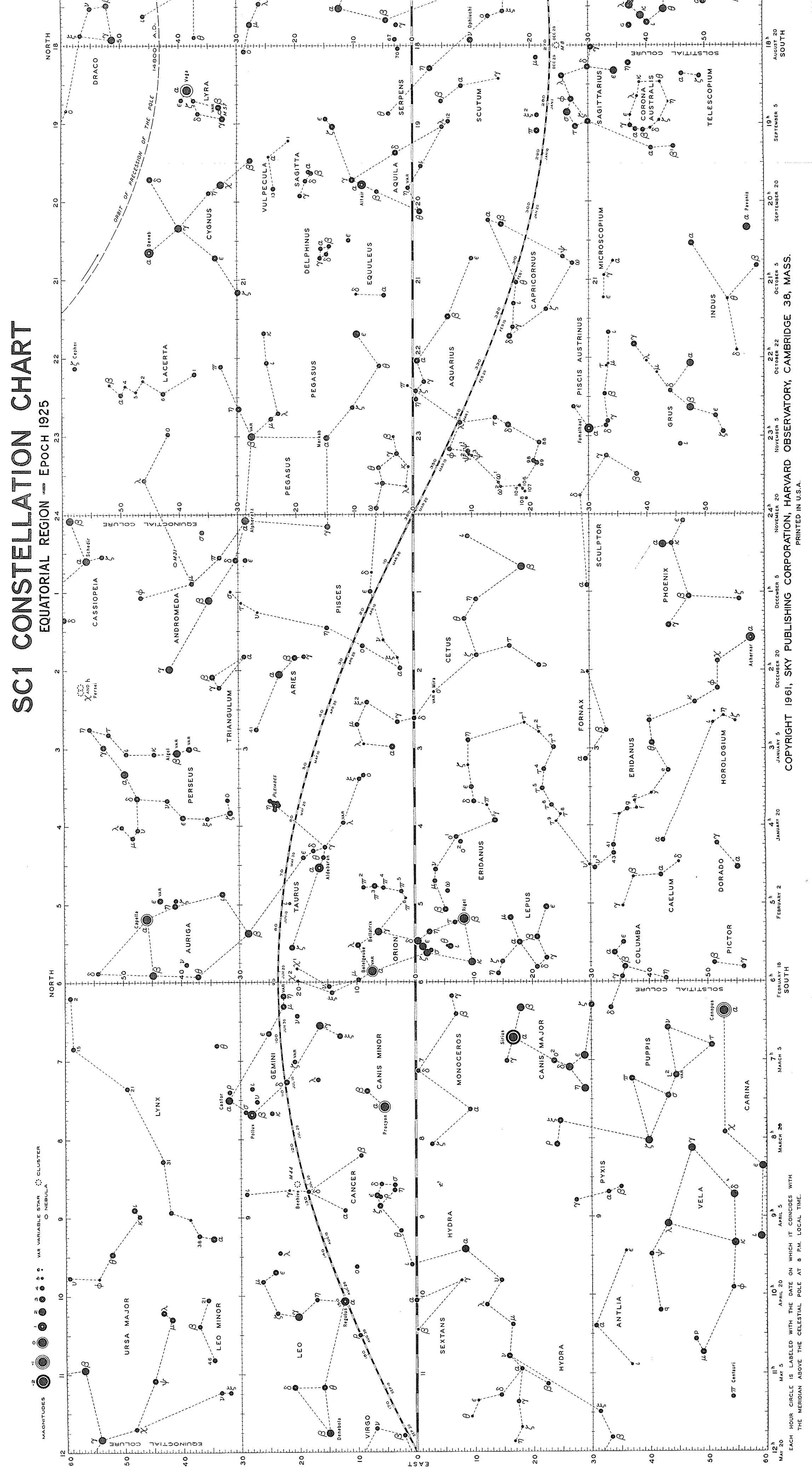




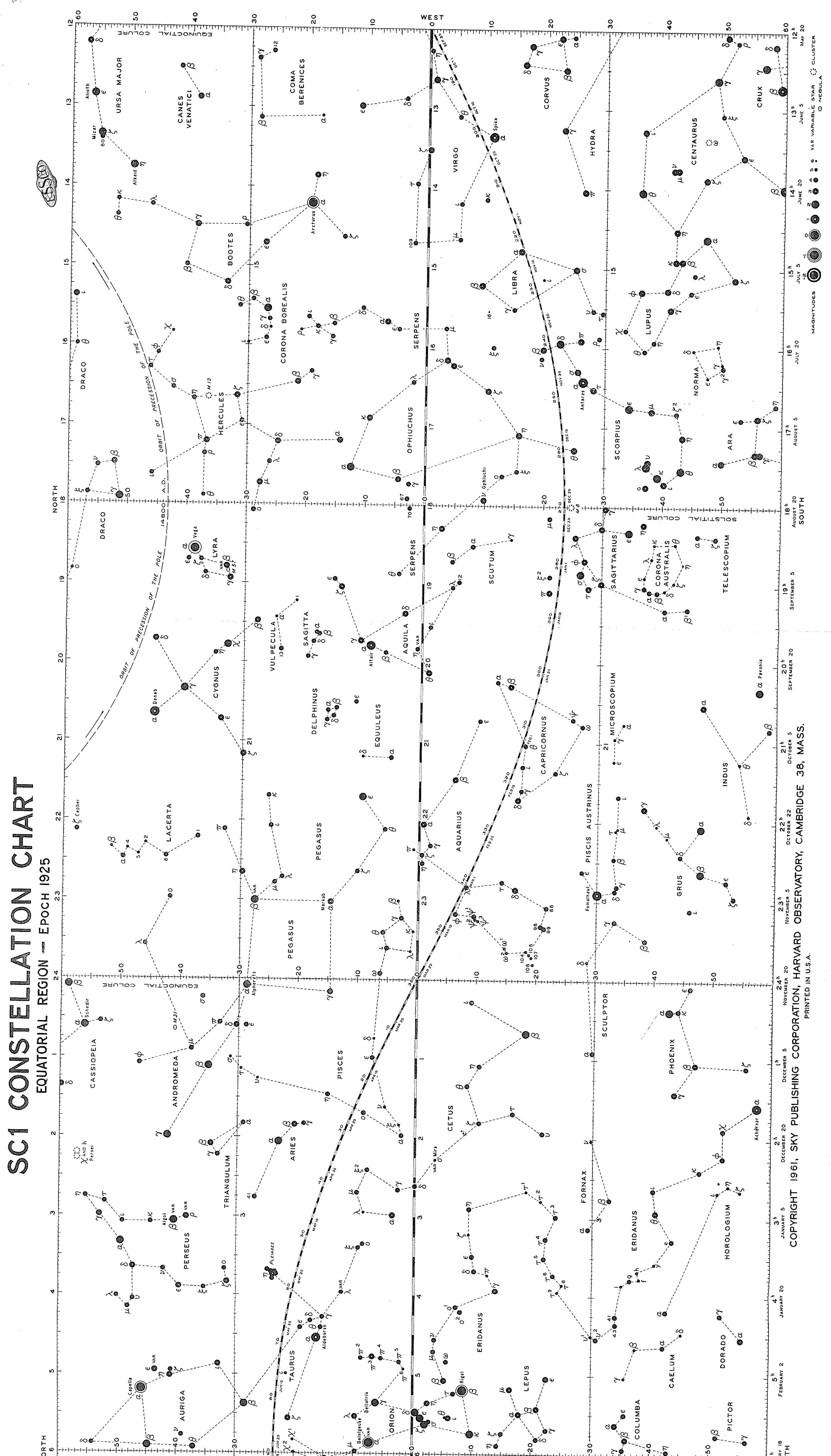


viewing directions proved unfortunate. It was considered at one time to have one telescope pointing into the Milky Way while the other telescope at right angles to it would be pointing into the shorter, much less dense arm of the galactic disk. In fact, this is a possible future experiment.

A solar drive, on the other hand, does not necessarily require a judicious choice of viewing directions, as during the course of a year's run, each telescope slowly scans the entire celestial equator. In addition, a solar drive by its very nature is equipped to study possible influences of the inner solar system on the arrival directions of the primary cosmic particles. With this type of rotation directions are defined in relation to the PES line. Figure 3 shows the PES line as observed looking vertically down onto the north pole of the earth, and indicates the manner in which the directions 'east' and 'west' are measured with respect to this line.

The experimental arrangement was therefore a pair of geometrically identical telescopes rotating by means of a solar drive in the manner described above. This arrangement is such that six hours after one telescope has a given orientation with respect to the laboratory (and therefore the earth) the other telescope has exactly the same orientation with respect to the earth's atmosphere and magnetic field. Each telescope is bi-directional. However, in all but the 
FIGURE 3

The PES Line 


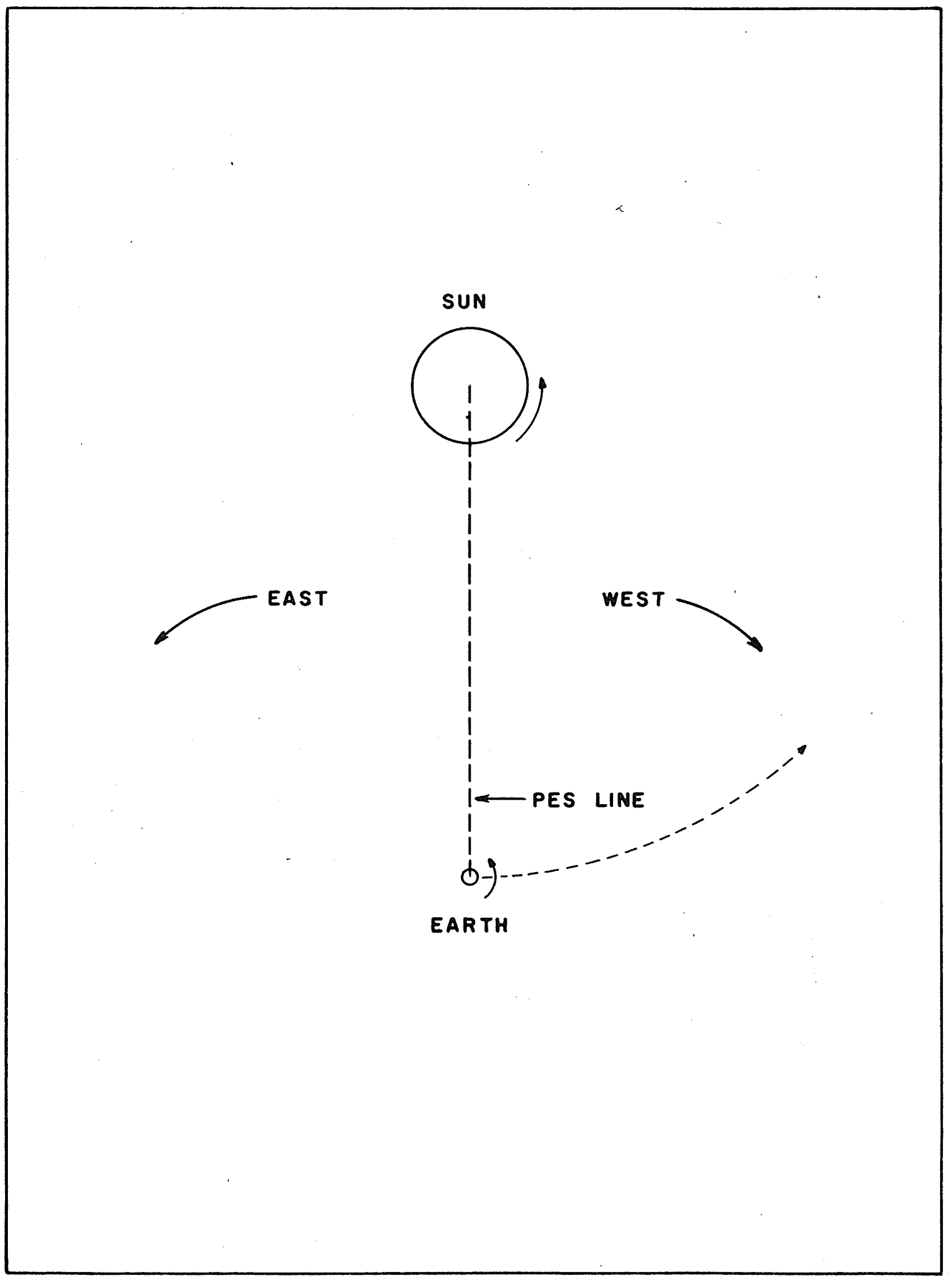


horizontal position (where the counting rate is close to zero anyway) the earth acts as an infinite absorber and thus during any one day the telescope effectively views two distinct and opposite directions relative to the PES line, alternately observing each direction for a twelve hour period.

It is estimated (See Appendix I) that the telescopes utilized in this research detect $\mu$-mesons produced by primary protons of energy greater than about $40 \mathrm{Bev}$ with an average energy of about $120 \mathrm{Bev}$. The detected muons approximately preserve the direction of the primary parent momentum. Although the telescope continuously points in the same direction relative to the PES line in the plane of the celestial equator, it is evident that due to its daily rotation about an axis parallel to that of the earth's, its orientation with respect to a set of reference laboratory co-ordinates will be a function of time, and in fact, a telescope will continuously trace out the same sequence of laboratory orientations. Because of the symmetry of the rotation, as the telescope rotates from the horizontal plane to the vertical plane it passes through a succession of zenithazimuth angle combinations which are then exactly reversed as it moves back down again to the horizontal plane. The values of zenith and azimuth combinations as a function of telescope rotation are calculated in Appendix II. The theoretical values 
obtained were checked by several series of direct measurements. Another contrasting feature of the rotating meson telescope with a stationary telescope is described in terms of the associated asymptotic cones of acceptance. Whereas a telescope rigidly fixed to the earth's surface constantly samples radiation through a single 'sky window', the rotating telescope, due to its constantly changing succession of zenithazimuth angle orientations in the earth's reference frame, observes radiation from a repeating pattern of sky windows, one such window associated with each particular orientation. Thus, a stationary telescope may be considered to be always looking through the same window at a variety of directions, while the rotating telescope al ways looks at the same direction in space through a variety of windows.

The physical and electronic aspects of the construction of the rotating high energy particle telescopes are considered in the next section of this thesis. 
$\underline{P A R} T \underline{T H R E}$

DESIGN AND PERFORMANCE OF THE EQUIPMENT 


\subsection{THE CONSTRUCTION OF THE COSMIC RAY TELESCOPE}

In the astronomical studies of the galaxy, telescopes are mounted in such a manner that they can turn on two mutually perpendicular axes to follow the circles of the equatorial system of the galactic sphere. These two axes are termed the polar axis and the declination axis respectively, the rotation of the former being used to locate the diurnal circle of a particular celestial body (Right Ascention, RA) and the latter the declination of the body.

A polar axis is simply an axis on the surface of the earth which is parallel to the axis of the earth. For observers in the northern hemisphere the north celestial pole (the intersection of the projection of the earth's axis with the celestial sphere) is marked to within less than a degree by Polaris, the pole or north star, at the end of the handle of the Little Dipper (Ursa Minor).

As a polar axis is parallel to the earth's axis, it is therefore inclined to the horizontal at an angle equal to the geographic latitude of its location. Around this axis the telescope may be turned parallel to the celestial equator and so along the diurnal circle of a particular star. The declination axis is supported by the polar axis (at right angles), and around this axis the telescope may be turned along an hour circle (i.e. from one declination to another).

Figures 4 and 5 schematically show the large equatorial 
FIGURE 4

The Main Sections of the Equatorial Mount 


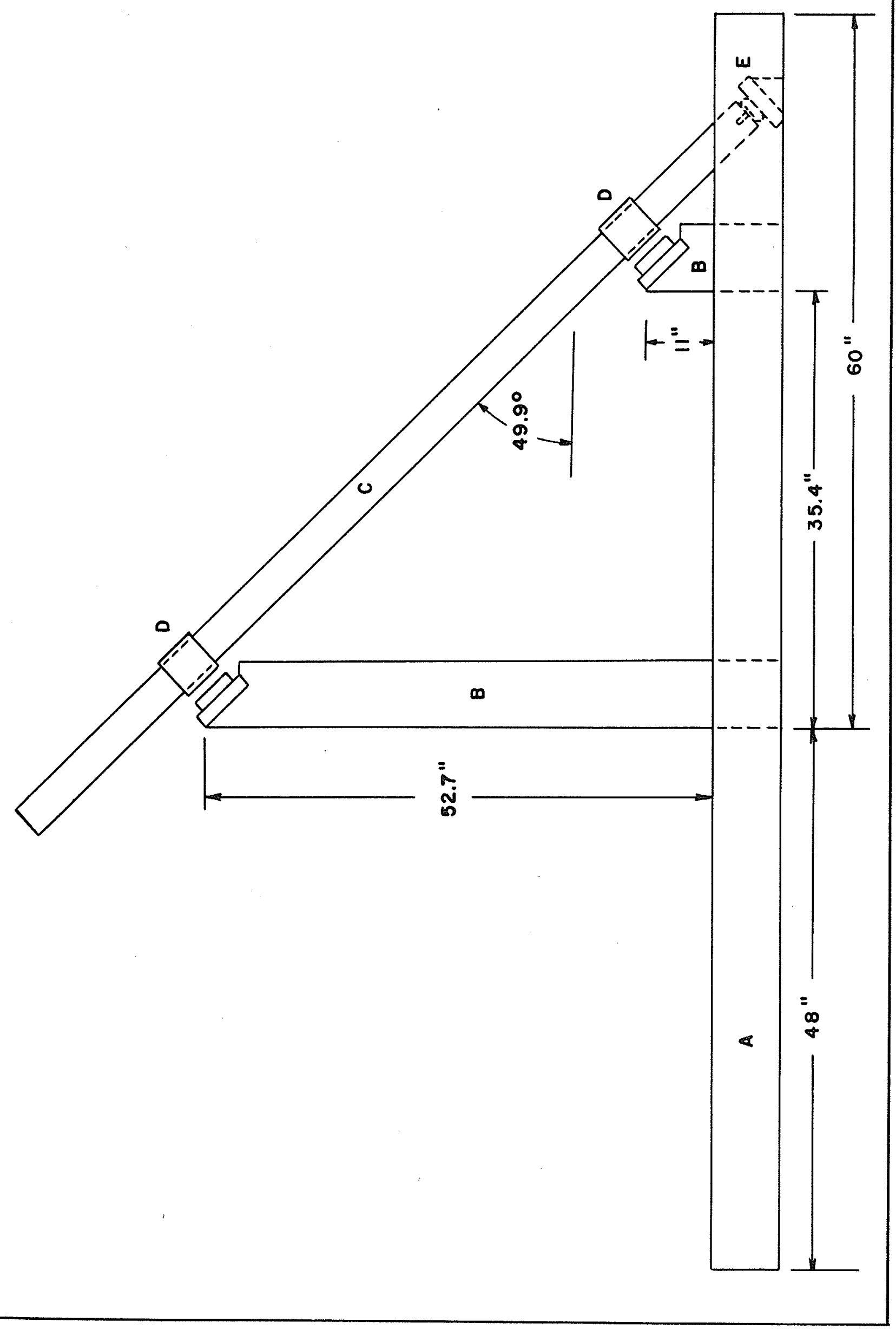


FIGURE 5

Construction Details of the Base of the Equatorial Mount 


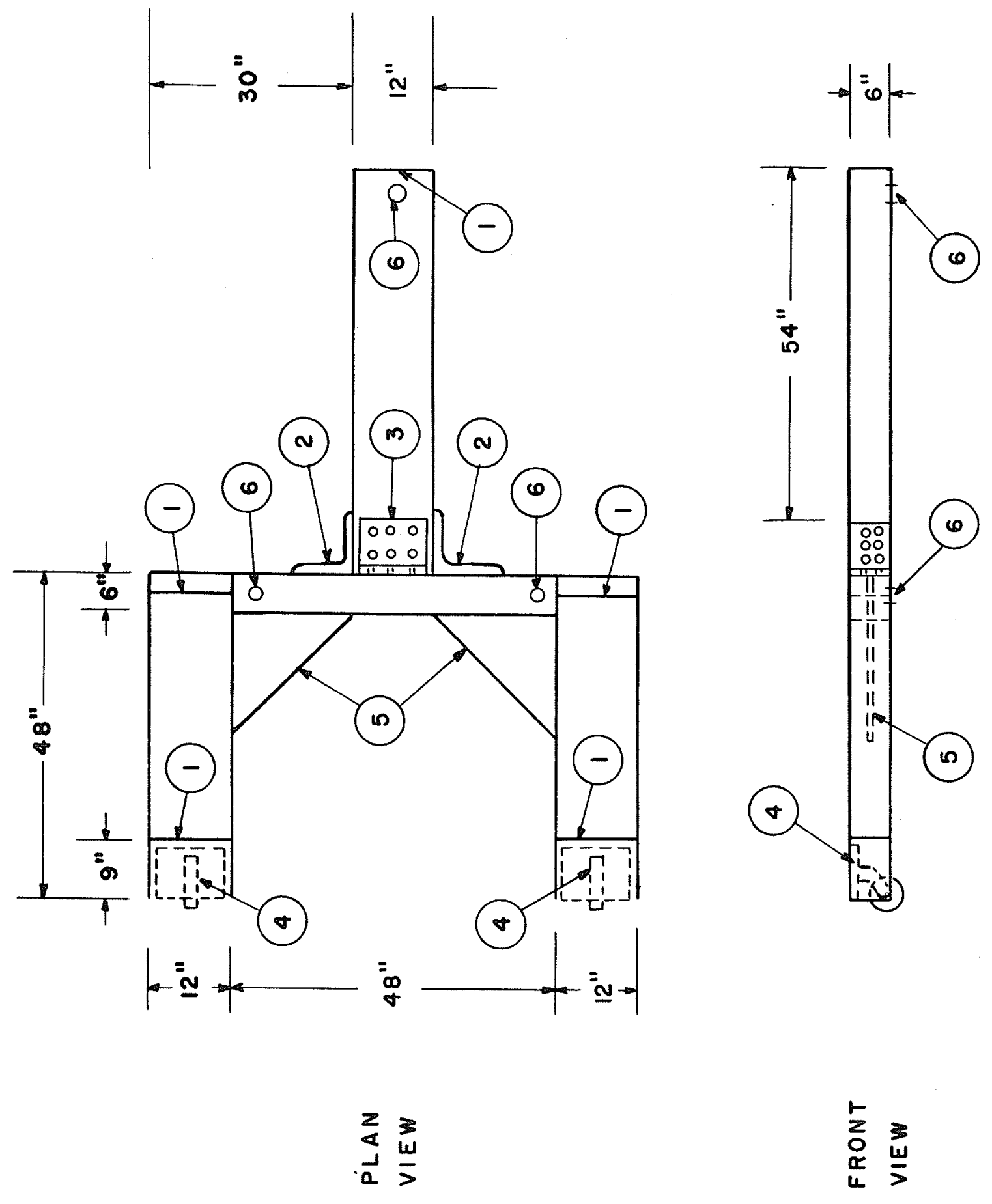


mount designed and constructed for the current research. The entire framework was constructed from $3 / 8$ cast iron beams machined into a cross-sectional $U$-shape for structural rigidity. The large surface area ( 6 feet by $81 / 2$ feet) which the completed unit occupied ensured that the flooring upon which it rested was subjected to allowable pressure. When fully loaded the total assembly weighed about 1500 pounds.

The astronomical mount consisted of three main sections: a horizontal I--shaped base, two vertical supporting beams for the polar axis, and the polar axis.

A detailed design diagram of the base is shown in figure 5. The overall dimensions of the base were $6^{\circ} \times 81 / 2^{\circ} \times 1 / 2^{\circ}$ deep. It consisted of two sections $48 " \times 12 \% \times 6 " \times 3 / 8 "$, one section $48^{\prime \prime} \times 6 " \times 3 / 8^{\prime \prime}$ and one section $54^{\prime \prime} \times 12^{\prime \prime} \times 6 "$ $x$ 3/8\%. Various features of the design are indicated in the figure by reference circles. Reference 1 depicts five pieces of $111 / 4 " \times 53 / 4 \% 1 / 4 \%$ sheet metal plate, flame cut, and welded into position in the locations shown. Reference 2 refers to two pieces of $6 " \times 6 \times 5 " \times 3 / 8 "$ angle iron each containing 8 holes drilled to accommodate $1 / 2$ " Allen cap screws. These angle iron frames reinforced the junction of the base and the larger vertical beam. Reference 3 illustrates a $6 " \times 6 \% \times 1 / 2 * 3 / 8 "$ angle iron frame containing twelve $1 / 2^{\prime \prime}$ holes, also used to reinforce the vertical beam. Reference 4 indicates two metal casters, $41 / 2^{\prime \prime}$ in diameter, each mounted 
on a $111 / 4 " \times 9 \% \times 1 "$ mild steel plate. These casters provided ease in the physical movement of the assembly. Reference 5 shows two pieces of $2 \pi \times I^{n}$ channel iron arc welded into position to add additional strength and rigidity. Reference 6 indicates three one inch diameter threaded holes into which were inserted threaded steel jacking pads whose function was the fine levelling adjustment needed to ensure that the base was horizontal. Levelling was accomplished by means of several long carpenter's bubble levels, and the consistency of this levelling was periodically checked.

Figure 4 shows the dimensions and relative locations of of the two vertical beams rigidly attached by means of $1 / 2^{\pi}$ bolts to the base of the equatorial mount. This figure also shows the manner in which the vertical beams support the polar axis at an angle of $49.9^{\circ}$ (the geographic latitude of Winnipeg) to the horizontal. The vertical beams were likewise constructed of $6 " \times 6 " \times 3 / 8^{*}$ channel iron with the leading edge of the front beam being 58.7 inches in length and the leading edge of the back beam being 17 inches in length. The centres of these two vertical support beams were 35.4 inches apart. Each vertical section supported a carefully ground bearing mount which in turn supported the bearing block through which the polar axis was enabled to rotate. All surfaces were machined to a tolerance of about .005\%. Each bearing plate was machined from mild steel to be $131 / 4^{\prime \prime} \times 53 / 4^{*} \times 1 "$ and

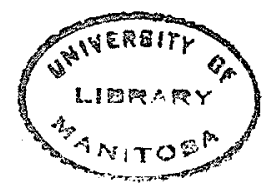


contained two $7 / 8 \pi$ diameter holes for mounting purposes.

The polar axis used was a shaft of mild ground steel, being $91 / 2^{\prime}$ in length, circular in cross-section with a diameter of $3^{\pi}$, and weighing about 240 pounds. Contact between the polar axis and the equatorial mounting frame was made at three locations, twice through self-aligning bearings and finally at a specially constructed thrust plate at the base of the mount. These are indicated at $D$ and $E$ in figure 40 The self-aligning bearing blocks used were SKF pillow blocks which came equipped with flanged mountings and were large enough to accommodate the three inch shaft. The thrust plate was constructed of mild steel and consisted of a $6 " \times 7^{n} \times I^{n}$ face plate mounted on two triangular blocks of altitude $33 / 4 \%$, angle of $40.1^{\circ}$ and thickness of $I \%$. A one inch hole was drilled into the bottom face of the polar shaft into which was inserted a rod welded onto a thrust bearing. The thrust bearing was in turn forced flush against the face of the thrust plate. This feature is also shown schematically at $E$ in figure 4. All surfaces were ground and polished carefully and considerable care was taken in both the cutting of angles and the arc welding of joints to form a particular angle.

The telescopes were rigidly attached to the polar axis by means of a specially constructed mount to accommodate a universal swivel plate assembly. The mount and assembly consisted of two circular disks $10^{\text {" }}$ in diameter and $I^{\text {" thick }}$ 
separated by two parallel mounting plates $6 \times 8 \% \times 1 "$ con-

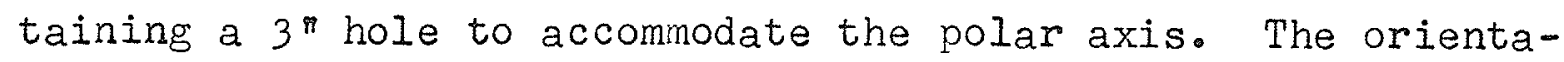
tion of this unit was such that the mounting plates were welded into location perpendicular to the polar axis with each circular disk welded to the plates in a plane parallel to the direction of the polar axis and 3 " away from its centre. To one of the circular disks was affixed the telescope arm and to the other was affixed a counterbalance arm. The salient features of the telescope mounting are depicted in plate 1.

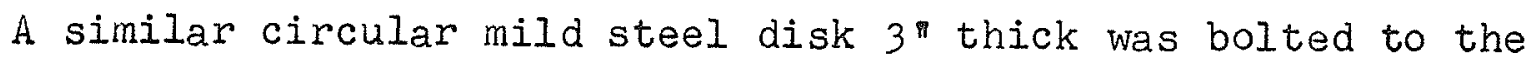
centre of the telescope and served as the other half of the universal swivel plate assembly. The principle of operation of this assembly was as follows: The circular disk rigidly fixed to the polar axis contained four cut away sections lying along a circle of 4 "radius and equally spaced along this circle. The circular disk rigidly fixed to the telescope proper contained twelve threaded $1 / 2$ " holes tapped $I "$ deep at $30^{\circ}$ intervals along the $4^{\prime \prime}$ radius curve to accommodate 1/2" Allen cap screws. Thus, by a physical rotation of the telescope with the two circular faces flush, the holes in the disk on the telescope could be brought into alignment with the cut-outs on the disk attached to the polar axis and the telescope then bolted securely by means of the large $1 / 2^{\pi}$ Allen cap screws. In such a manner the telescope could be set at any desired declination angle. For the current research, 
PLATE 1

Mounting Junction and Universal Swivel Plate 


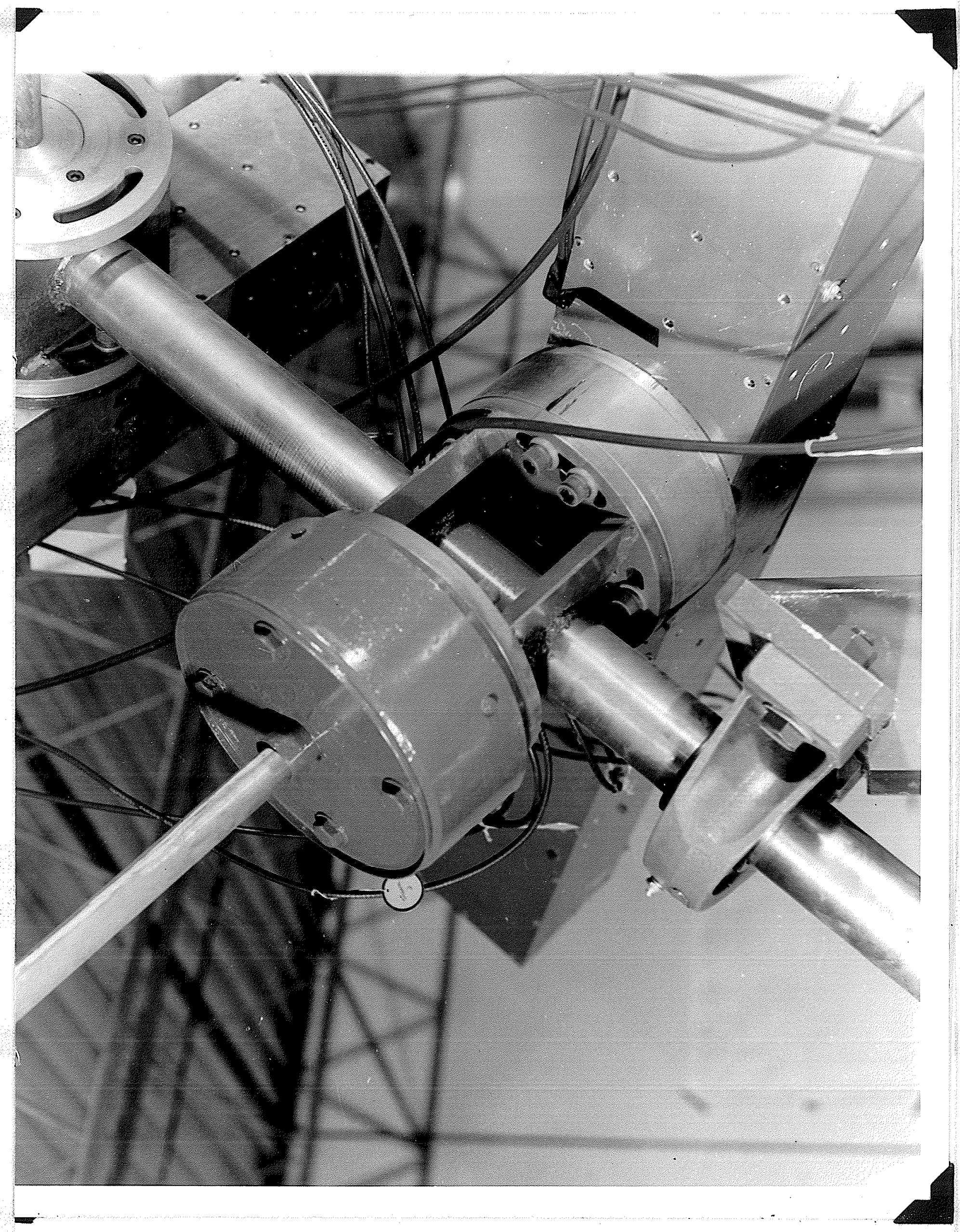


however, the declination chosen was $0^{\circ}$ (i.e. the telescope was directed towards the celestial equator) and hence that combination of tapped holes was chosen such that the telescope was mounted at right angles to the polar axis. Two such swivel assemblies were constructed and arc welded onto the polar axis at right angles to one another (The right angles were aligned with the aid of an engineer's transit). Thus, in the final arrangement the two telescopes and the polar axis formed a right triad, with the polar axis inclined at an angle of $49.9^{\circ}$ to the horizontal in the laboratory system.

Each of the two telescopes was constructed from $1 / 2^{\prime \prime}$ mild steel beams welded together to form long rectangular blocks of inside cross-section $6 n \times 6 \%$. Due to space limitations existing within the laboratory, the two telescopes were constructed of different lengths, one being $77^{\circ}$ and the other $31^{\%}$. The basic properties of each were the same, however, each being hollow and each being designed with $3 / 8$ " aluminum plates acting as spacers to define internal compartments within the telescope. These spacers were easily moved, being fixed to the telescope frame by means of $1 / 4 \pi$ Allen cap screws. Several sets of small. holes drilled into the tube defined the location of these spacers and others could be drilled in the future with minimum effort should it become desirable to change the sizes of the compartments. In the current investigation three compartments 
were required in each telescope, the middle compartment containing $\mathrm{Pb}$ absorber and the outer two compartments housing the phosphor detector heads.

The $\mathrm{Pb}$ absorber was poured and moulded into cylindrical blocks 5 " in diameter and of variable lengths from $I$ " to 6 " thereby offering a wide selection in the amount of absorber desired. Cylindrical wooden blocks $5 "$ in diameter and $I "$ deep were constructed which could be inserted into the compartments. This enabled still smaller solid angles without the introduction of more absorber.

The detecting phosphors with their mounted photomultiplier tubes were held firmly in place in their compartments by wooden collars which were cut and shaped to fit snuggly around the detectors in such a manner that the phosphors were exactly centred in the compartment. The same applied to the $\mathrm{Pb} c y \mathrm{c}-$ inders which were inserted between two sections of $6 \pi \times 6 \pi$ wooden blocks into which were hollowed 5 " diameter holes.

Access to the compartments was accomplished through the front section of each telescope which was constructed in five interlocking segments held to the framework by Allen cap screws. Holes were also cut in the sections that covered the phosphors to allow the necessary conducting cables to reach the base of the photomultipliers.

Each counterbalance arm was constructed from a $2 "$ mild steel shaft one end of which was threaded to accommodate cylindrical $\mathrm{Pb}$ counter-weights, while the other end was anchored 
in a lead filled oil drum which was firmly bolted to the swivel plate mount opposite to the telescope proper. The counterbalance weights were adjusted until the system was both statically and dynamically balanced.

The polar axis was rotated once per solar day in a laboratory direction of east to west (i.e. in a sense opposite to that of the earth's daily rotation) by means of a solar drive unit which was assembled and mounted on the side of the equatorial mount. The driving torque was supplied by a $72 \mathrm{rpm}$ Slo-Syn motor operating on the 60 cycle power mains. This rotation was reduced to a speed of 1 revolution per solar day by means of a gear-reduction box of ratio $540: 1$, a worm gear assembly of ratio $72: 1$, a set of spur gears of ratio 15:20, and finally a drive crown and pinion arrangement of ratio $32: 9$.

Plate 2 shows the completed, fully loaded equatorial mount with the two telescopes mounted at right angles to one another and at right angles to the polar axis.

After the entire assembly was completely constructed and loaded, the polar axis was aligned so as to be directed towards the North Star. The procedure utilized is described in Appendix III. 


\section{PLATE 2}

Fully Loaded Operative $\mu-$ Meson Telescopes 


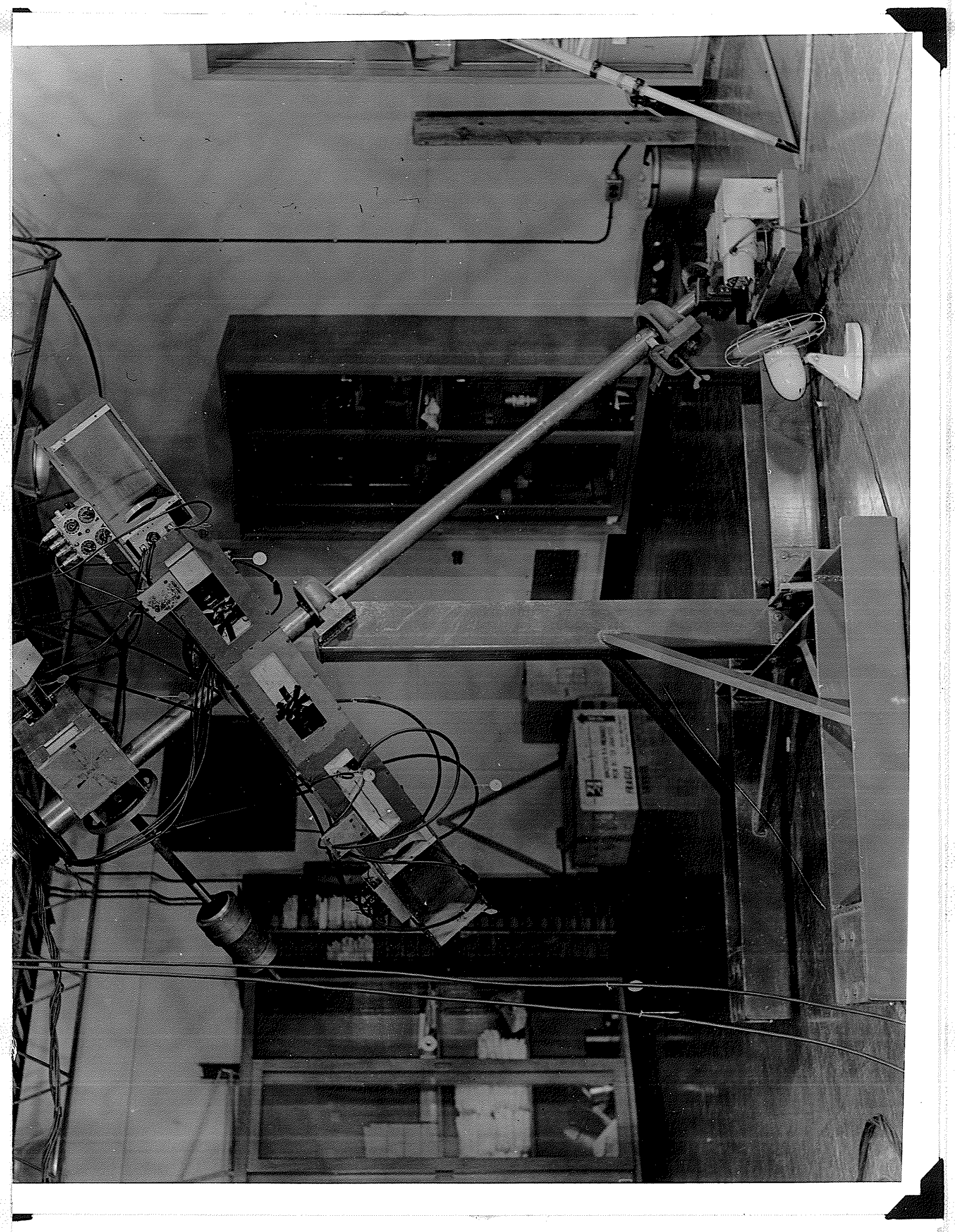


3.2 THE TELESCOPE GEOMETRY

Each telescope contained two scintillation phosphors separated by a finite amount of $\mathrm{Pb}$ absorber. Each detector was cylindrical in shape, $8.89 \mathrm{~cm}$. in diameter and $5.08 \mathrm{~cm}$. thick. Mounted on each detector was a 5 " DuMont photomultiplier tube firmly affixed with viscous silicone grease and rendered light-tight by a liberal application of Apiezon $Q$ compound and black electrical tape. The outputs of the two detectors were then taken in coincidence.

The geometry of the long-term experimental run (March 1963 to November 1963) is shown in figure 6(a). During this time the detectors were separated by $22^{n}(55.9 \mathrm{~cm})$ of $\mathrm{Pb}$ and the detectors subtended a cone of half angle $7.7^{\circ}$.

The coincidence solid angle, however, was the angle $\alpha$ indicated in figure 6(b). A particle may travel anywhere within the solid angle $\alpha$ and still pass through both detectors and hence register a two-fold coincidence. The value of $\alpha$ is, of course, a function of the location of the apex of the angle on the top detector. However, for the geometrical arrangement shown, the values of $\alpha$ for the apex taken on the edge of the detector and for the apex taken at the centre of the detector differed by only $0.28 \%$. Hence an average value of 0.01422 sterads was taken.

The function of the lead absorber was two-fold: First, it ensured that the electrons present in the secondary cosmic 
FIGURE 6

Geometry for Narrow Angle Run

(a) Telescope Angle

(b) Coincidence Angle 


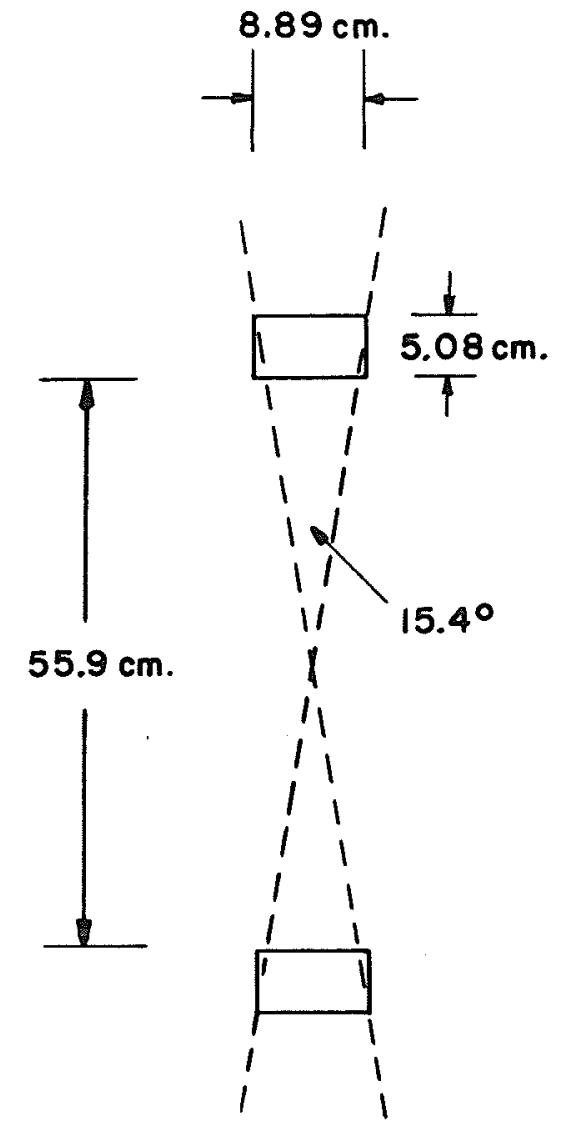

(a) TELESCOPE ANGLE

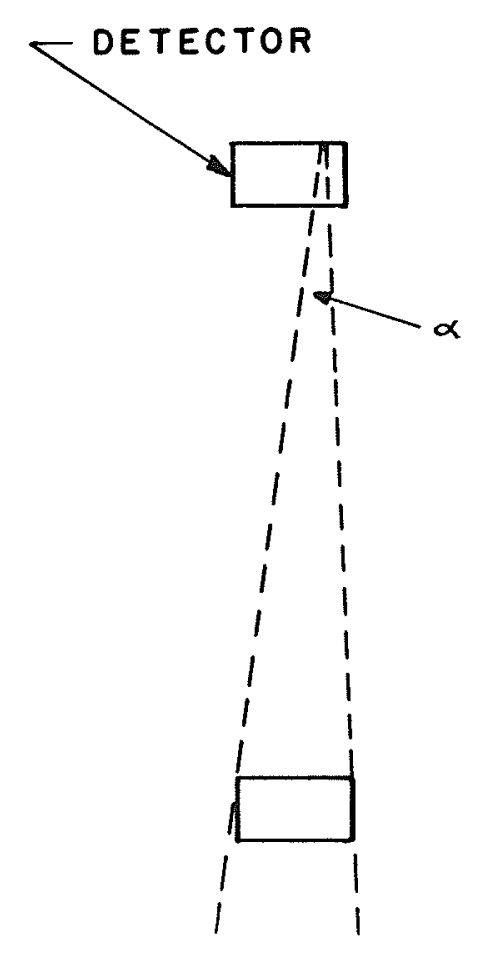

(b) COINCIDENCE ANGLE 
radiation could not penetrate to the second detector (about $10 \mathrm{~cm}$. is sufficient to ensure that the fast interacting electrons are filtered out of the beam). Thus only mesons and protons would effect a coincidence. Second, the lead absorber set a lower limit on the energies of the penetrating particles that did reach the second detector.

For the experimental data collected beyond December 18 , 1963, the geometry of the detector arrangement was changed. Exactly half the $\mathrm{Pb}$ absorber was removed, leaving II" separating the phosphor pairs. This resulted in the detectors subtending a cone of half angle $13.1^{\circ}$ while the coincidence solid angle was increased by a factor of $\omega 3$ to 0.04266 sterads.

Even though both geometries define relatively narrow telescope angles, in order to distinguish between them, experimental runs taken before December 18 will be referred to as 'narrow angle' runs, while those taken after December 18 will be termed 'wide angle' runs.

\subsection{THE SCINTILLATION DETECTORS}

Although the telescopes were constructed in such a manner that each was geometrically identical to the other, each employed different scintillators as its detector heads. One telescope utilized two NaI(TI) crystals while the other utilized two NE102 plastic phosphors (polyvenyltolvene). As 
these scintillating materials display distinct properties when subjected to impinging radiation, it was necessary to employ different energy discrimination levels for each system。

The choice of an integral energy discrimination level for the $\mathrm{NaI}(\mathrm{TI})$ crystals was a relatively simple operation, while that for the plastic detectors presented a bit more difficulty.

Figure 7 shows a sample high energy cosmic ray spectrum near sea level taken with one of the $\mathrm{NaI}(\mathrm{TI})$ crystals and obtained on a 100 channel pulse height analyzer (Computing Devices of Canada Ltd.). The outstanding feature of this spectrum is the broad peak which is predominently the result of the deposit of energy by cosmic ray $\mu$-mesons in their passage through the detector.

Since the photopeak of a low energy $\gamma$-source spectrum is a distinguishing feature of the spectrum when obtained with a $\mathrm{NaI}(\mathrm{TI})$ phosphor, the energy of such a photopeak may readily be used as an energy calibration for the $\mu$-meson peak obtained from the same crystal. Using the $1.12 \mathrm{MeV}$ $\mathrm{Zn}^{65}$ photopeak as the calibrating energy, standard scintillation counting techniques yielded an experimental value of about $25.3 \mathrm{Mev}$ for the $\mu$-peak. Thus, on the average, a $\mu$-meson loses about $12.6 \mathrm{Mev}$ of energy per inch of $\mathrm{NaI}(\mathrm{TI})$ detector traversed. In order to include those muons that contributed 


\section{FIGURE 7}

Sample High Energy Cosmic Ray Spectrum Neax Sea Level 


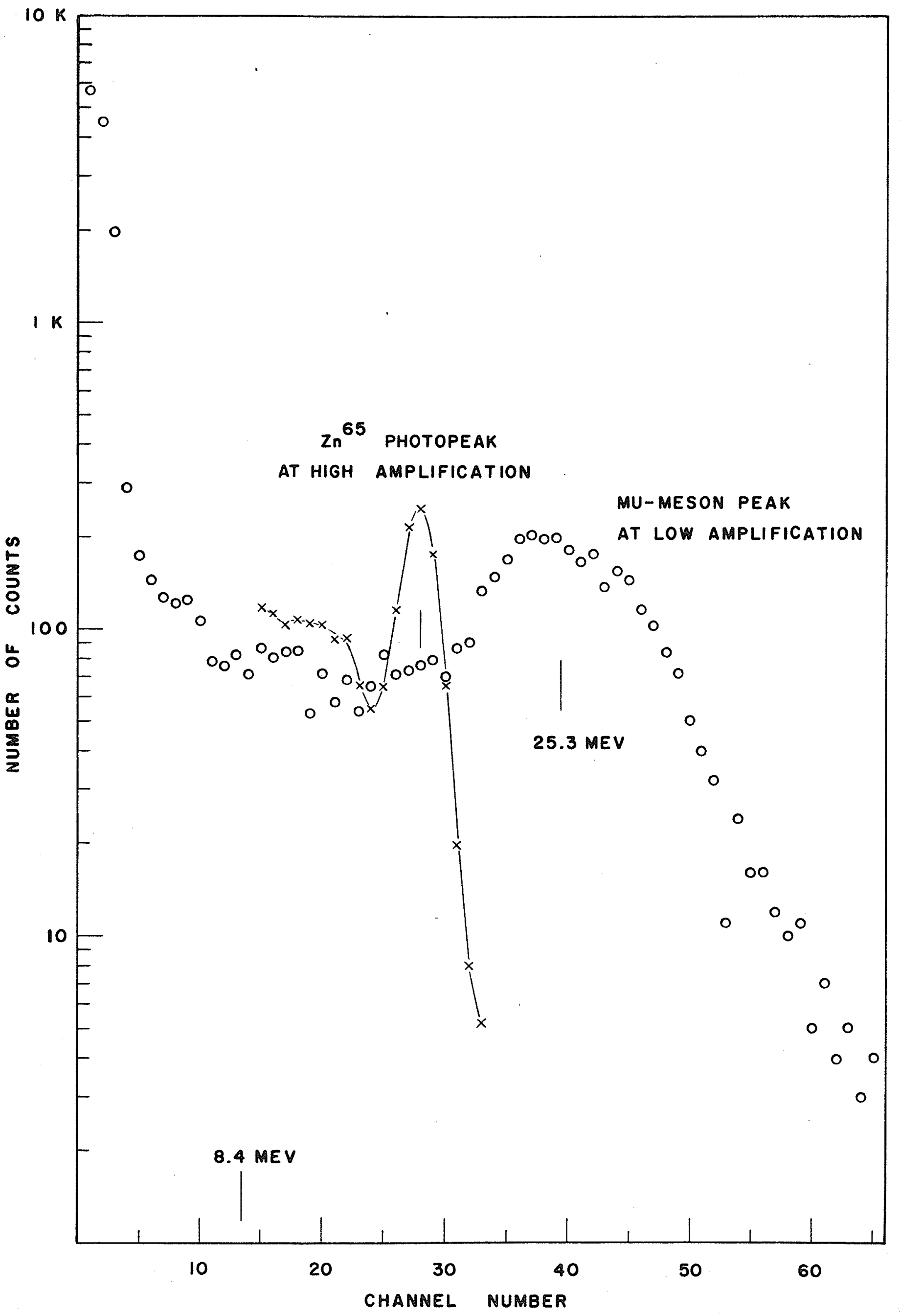


to the valley of the $\mu$-peak, an integral discriminator value of $8.4 \mathrm{Mev}$ (as shown in figure 7) was chosen. This discriminator value of $8.4 \mathrm{Mev}$ yielded a counting rate for the telescope in the vertical orientation which agreed with the expected vertical counting rate. The vertical flux of muons appropriate to the detectors was taken (Puppi, 1956) to be about $6.3 \times 10^{-3} \mathrm{~cm}^{-2}$ sterad $^{-1} \mathrm{sec}^{-1}$. For the telescope geometry: the expected vertical counting rate was calculated as 20.0 counts per hour. With the discriminator levels set at 8.4 Mev for the $\mathrm{NaI}(\mathrm{TI})$ crystals, this telescope in 76 hours of counting time recorded $20.1 \pm 0.5$ counts per hour. The cosmic ray spectrum obtained by the NEIO2 phosphors displayed essentially the same broad $\mu$-meson peak. However, for such a low $Z$ phosphor, the cross-section for impinging photons is very small and no photopeak is apparent, the photopeak being lost in the broad Compton peak. The discrimination levels for the NEIO2 plastics were determined by a trialand-error method. The levels were adjusted until the vertical counting rate of the telescope containing the plastics was brought into agreement with the vertical counting rate of the telescope containing the crystals. With the discrimination levels set at $\sim 4.8 \mathrm{Mev}$ for the NE1O2 plastics, this telescope in 77 hours of counting time recorded $20.1 \pm 0.4$ counts per hour. 
3.4 THE ELECTRONIC SYSTEM

A high energy $\mu$-meson arriving at the earth's surface from a particular known viewing direction in space was detected only after it had passed through the first detector head, penetrated the lead absorber, and entered the second detector depositing in it at least the discrimination level energy. The light flashes produced in the phosphors due to the impinging radiation were observed by the photomultipliers and converted into electrical pulses which were then fed into a coincidence circuit. Apart from accidentals, only those high energy particles travelling along the axis of the telescope within the coincidence solid angle were effective in producing a double coincidence pulse. Hence the detector pairs operated in coincidence directionally analyzed the impinging radiation.

A block diagram of one of the coincidence scintillation counter telescopes is given in figure 8. Two such circuits have been constructed, one for each telescope.

A hard tube cathode follower base was attached to the phototube and along with the detecting phosphor was mounted inside a telescope compartment. It thus rotated about the direction of the polar axis daily. The negative electrical output pulses from the cathode follower were then fed through sixty feet of RG59 conducting cable from the rotating telescope system to the attenuator located in the stationary main 
FIGURE 8

Block Diagram of the Electronies 


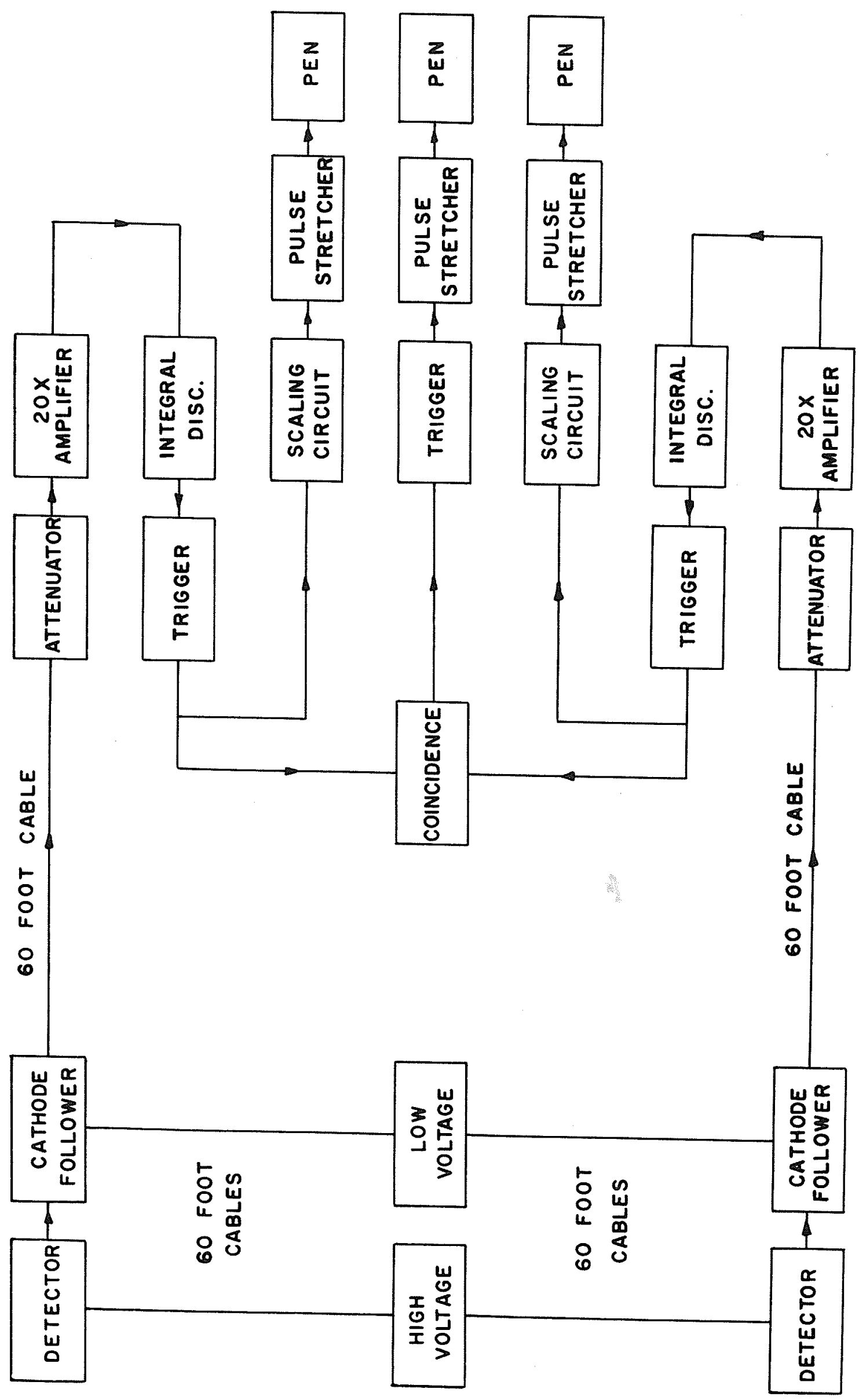


electronic rack. Upon arrival at the attenuator the average input pulses from a low energy $\gamma$-source were approximately 100 millivolts high. The average cosmic ray pulses were about 3 to 5 volts high with occasional very high pulses of about 10 volts. From the attenuator the pulses were fed through a fixed gain amplifier (20X) and then into an integral discriminator which was kept fixed at a level of 1 volt. The output pulse was then standardized to a clean positive going pulse 13 volts high and about 1 usec long by means of a Schmitt trigger circuit. The Schmitt trigger pulse was then fed simultaneously into both a coincidence unit and a pulse stretching unit via a scaling circuit. The stretched pulse was energized sufficiently to activate a recording pen of an Esterline-Angus event marker. The scaling circuit accepted 1024 pulses from the Schmitt trigger before a stretched pulse was fed to the pen. This pen then readily monitored the single events occuring in the phosphor.

The attenuators (100 ohm helipots), fixed gain amplifiers, discriminators, trigger circuits and coincidence circuits for all four detectors were included in one unit. This unit was completely transistorized and a schematic diagram tracing one channel is shown in figure 9. The coincidence unit which was just a simple diode adder circuit is shown by itself in figure 10. The pulses arriving at the diode were added, single pulses being too small to cause diode conduction, whereas 
FIGURE 9

Schematic Diagram of Single Channel of Transistorized circuit 


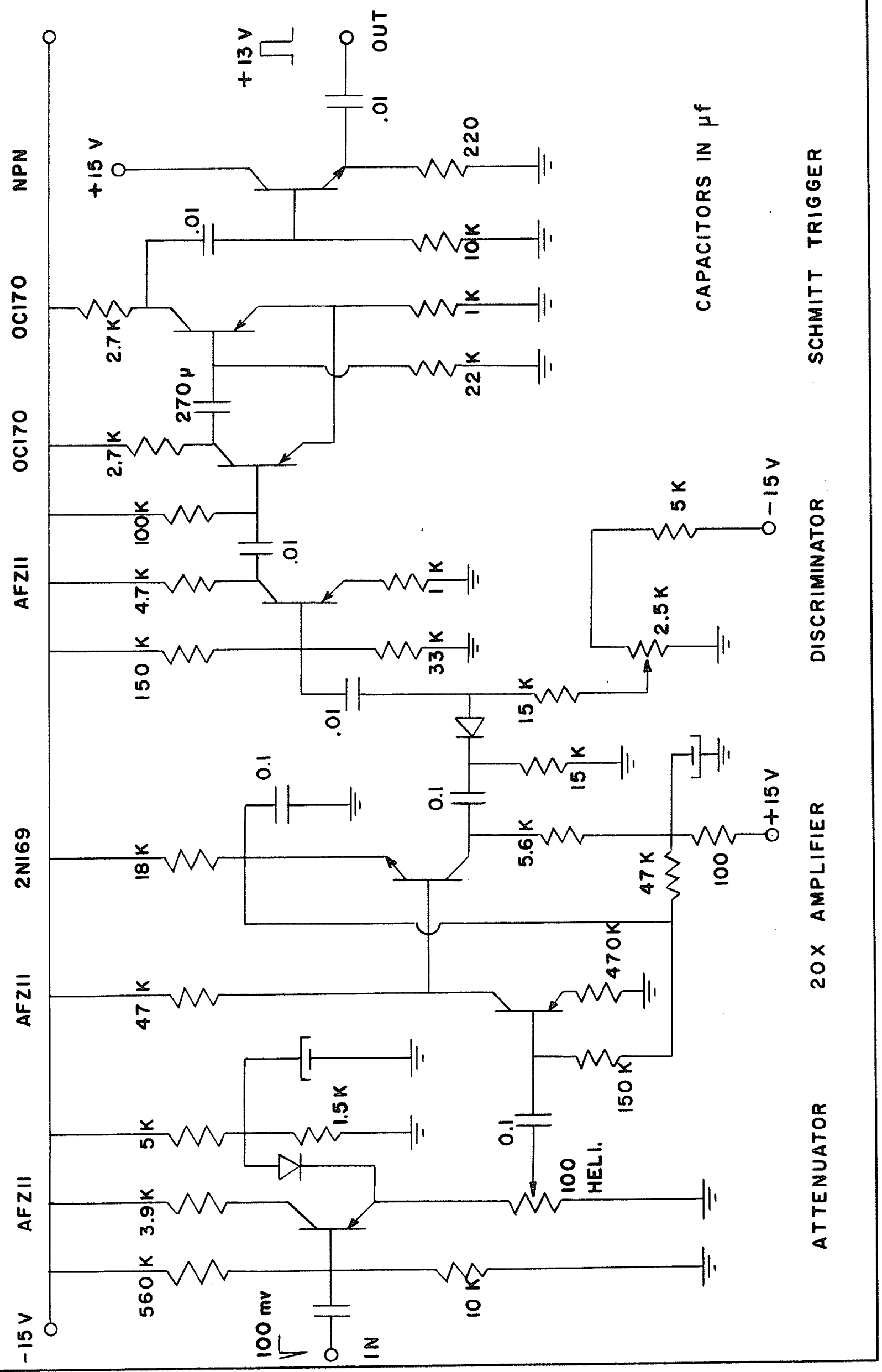


F IGURE 10

Schematic Diagram of Diode Adder Circuit 


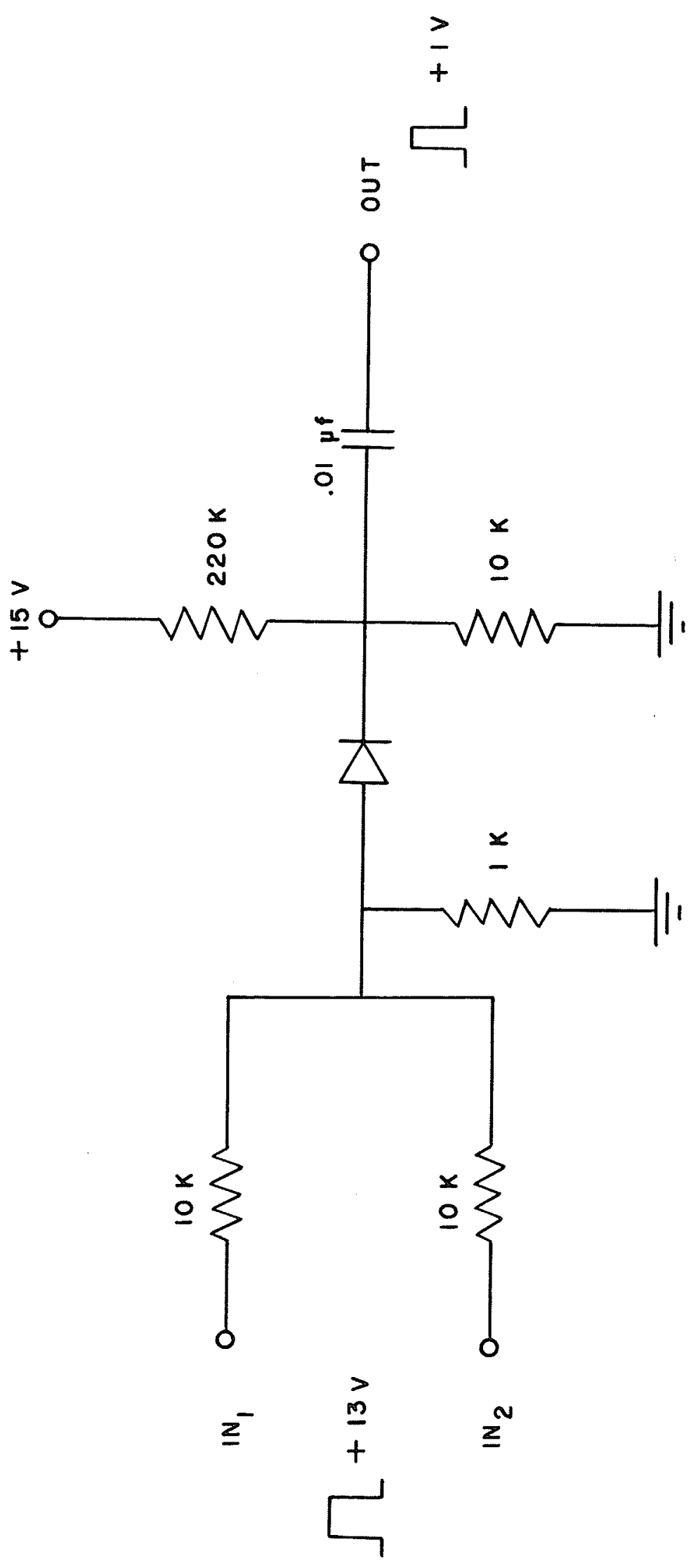


pulses arriving simultaneously from each of the two detectors produced a sum pulse which was large enough to cause diode conduction. The coincidence units had resolving times of approximately $3 \mu$ sec. However, for the single channel counting rates encountered in this investigation there was little need for faster coincidence circuitry (see below).

With the helipot attenuators set at scale readings such that the electronics discriminated against $8.4 \mathrm{Mev}$ pulses in the $\mathrm{NaI}(\mathrm{TI})$ detectors and against $4.8 \mathrm{NIev}$ pulses in the $\mathrm{NEIO} 2$ detectors, each of the $\mathrm{NaI}(\mathrm{TI})$ crystals in its daily rotation observed about 100,000 events, while each of the plastics observed about 138,000 events. Throughout the entire course of this investigation these single channel counting rates remained constant to within about $\pm 3 \%$. These counting rates resulted from the bombardment of the detectors by sea level cosmic radiation from all directions as the detector occupied all its orientations in moving daily in a circular path about the polar axis.

In the initial narrow angle runs the double coincidence rate for each pair of detectors was about 136 events per day, while with the wide angle telescopes this counting rate increased by a factor of nearly 3 to approximately 400 events per day. The $3 \mu \mathrm{sec}$ coincidence time introduced a random coincidence counting rate of about 0.7 counts per day in the telescope containing the crystals, while the telescope 
containing the plastics gave about 1.3 random coincidences per day。

In addition to a coincidence being triggered by the same particle travelling through both detectors, two individual particles simultaneously passing through each detector likewise instigated a coincidence. As a partial elimination of these unwanted coincidence pulses the following procedure was adopted: The predominant cause of these extraneous coincidences were shower particles. Hence for the condition of two particles passing through two of the detectors, there was a high probability that at least one of the remaining detectors would be likewise affected due to the proximity of all four detectors. With this consideration in mind, anticoincidence circuits were installed in the electronic system such that a pulse from either detector A or detector B (both located in one telescope) occuring simultaneously with a pulse from either detector $C$ or detector $D$ (both located in the other telescope) would prevent a coincidence occuring between detectors $C$ and $D$, and vice-versa. Thus the output pulses of both $A$ and $B$ served as blanking pulses for the coincidences $C D$ as did the output pulses of $C$ and $D$ for the coincidences $A B$.

The double coincidence pulses $A B$ and $C D$ were themselves fed into a coincidence adder circuit thereby effecting a monitor on 4 -fold coincidences $A B C D$. These 4 -fold coincidences 
presented some information on the frequency of showers, but since only about 6 to 7 such events occurred daily, no analysis were performed on these events.

In any case, the output pulses of the trigger circuits, whether single channel pulses, double coincidence pulses, or 4-fold coincidence pulses, were eventually stretched and fed to the pen recorder. In all, seven pens of the EsterlineAngus event marker were utilized, four pens recording the single channels $A, B, C$ and $D$, two pens recording the double coincidences, and one pen recording the quadruples.

The pen recorder charts were driven at a speed of $3^{\text {" }}$ per hour ( 6 feet per day) and the events recorded in all seven traces were read out manually. The relatively fast chart speed enabled the collected data to be analyzed very easily into twenty minute intervals.

High voltage to all four photomultipliers was provided from a single Hamner 2000 volt regulated power supply which passed the voltage down a single 60 foot RG59 cable from the stationary rack to four potentiometers mounted on the rotating scopes. This enabled each voltage to be adjusted independently of the other three. The high voltage to the NaI(TI) mounted phototubes was maintained at about 750 volts, while the high voltage to the plastic mounted tubes was maintained at about 1180 volts. Low voltage was delivered to the cathode follower tubes located within the bases of the 
photomultipliers in exactly the same way through 60 feet of No. 8643 six conductor shielded cable from a 250 volt power supply. All the units were operated from a voltage regulating Sorensen which received its power from a specially installed 120 volt 50 amp main.

All told, 6 sixty foot lengths of conducting cable formed the liaison between the rotating and stationary systems, two cables conducted the voltages to the telescope detectors and four cables conducted the output pulses from the rotating cathode followers to the transistorized circuits which analyzed them. The long conducting cables were bound together and wound around a spool fixed to the uppermost extremity of the polar axis. Thus, as the polar axis rotated, the cables unwound and the unwound cables were then directed out of the way of the subsequent rotation. The cables were rewound about the spool about twice a month.

Plate 3 shows the main electronic rack and data readout table containing the pen recorder (event marker).

\subsection{INITIAL ELECTRONIC ALIGNMENT AND CALIBRATION PROCEDURE}

The initial setting of the $8.4 \mathrm{Mev}$ and $4.8 \mathrm{Mev}$ discriminator levels on the $\mathrm{NaI}(\mathrm{TI})$ and $\mathrm{NEIO} 2$ detectors, respectively, accomplished by means of the variable attenuator, is herein described. The attenuator was situated in the electronic circuit immediately following the cathode follower and just 
PLATE 3

Electronic Control. Station 


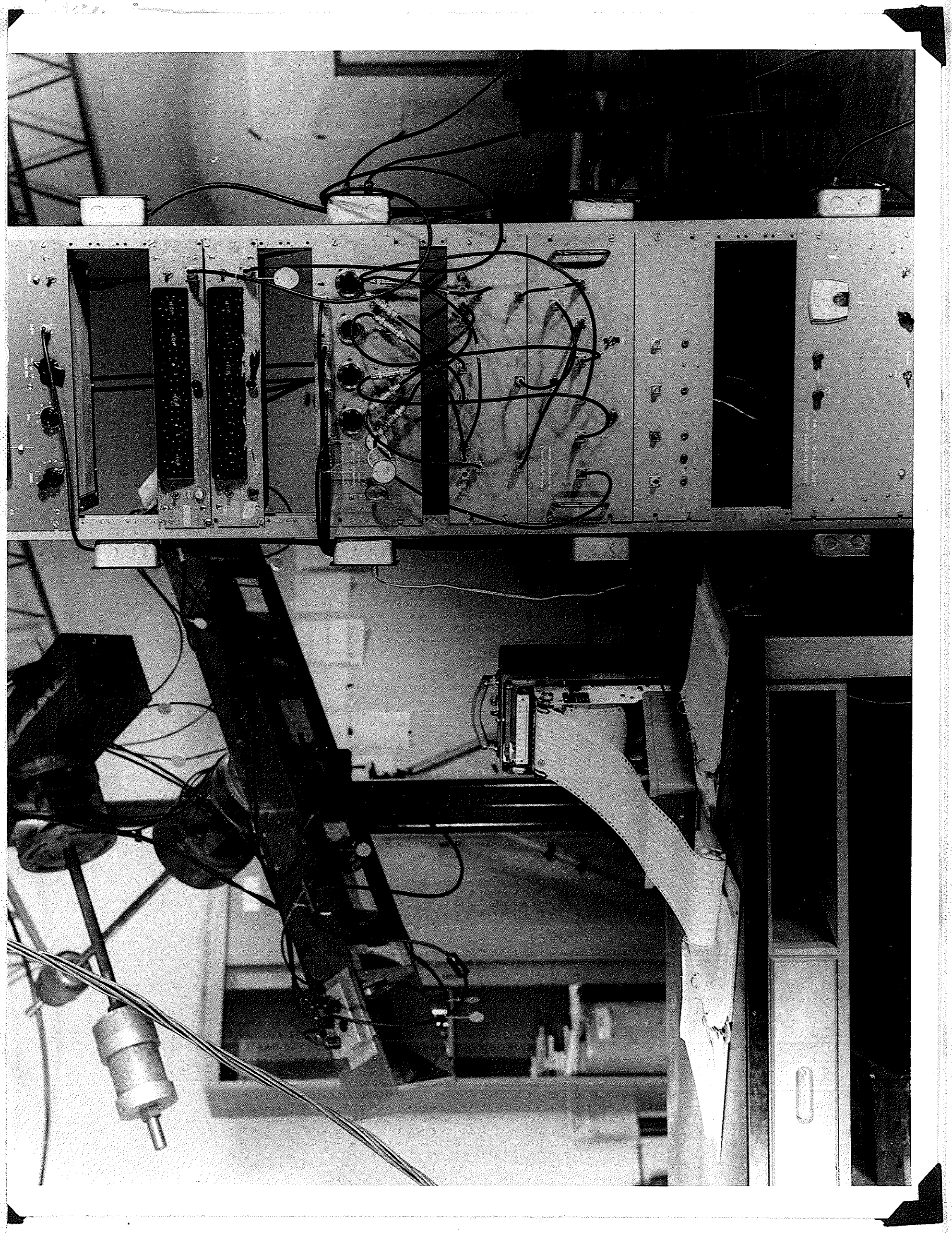


preceding the fixed gain amplifier. The integral level of the discriminator circuit was kept fixed at one volt. For the photomultiplier voltages used, the electrical pulses from a low energy gamma source ( $\mathrm{Na}^{22}$ ) were about $100 \mathrm{mv}$ high when they reached the attenuator from the cathode follower. Hence, with the helipot attenuator dial set at minimum attenuation (i.e. maximum amplification as the helipot dial was set to read degree of amplification rather than degree of attenuation) these small pulses corresponding to low energies could be amplified sufficiently to trigger the discriminator. As the attenuation was increased (i.e. the dial reading was reduced) a larger pulse from the cathode follower was required to effect a triggering of the discriminator. At high attenuation, therefore, the very much larger pulses required were provided by the high energy cosmic ray particles. In this manner energy discrimination was accomplished by the manipulation of the helipot dials in the electronic circuits of each detector.

The energy calibration of each helipot was a two-step procedure. First a spectral curve of counting rate versus helipot setting was obtained with a $\mathrm{Na}^{22}$ source mounted on the telescope frame at a fixed distance from the detector. Then the source was removed and a 'background' curve obtained. The high energy ( $>3 \mathrm{Mev}$ ) 'background' consisted of the cosmic radiation impinging upon the detector. The two curves 
obtained in this manner joined at some intermediate helipot setting and remained superimposed beyond this point as the attenuation was increased. This point corresponded to the maximum energy of the $\mathrm{Na}^{22} \gamma$-rays above background on the detector, and is therefore the high energy end of the photopeak distribution in the $\mathbb{N a}^{22}$ energy spectrum. This energy was taken as being 1.5 Mev. Thus, once this point had been obtained, simply taking ratios yielded the dial setting corresponding to any desired energy。

Figure 11 shows a sample line-up curve taken for one of the $\mathbb{N a I}(\mathrm{TI})$ detectors. Curve $S$ was obtained with the $\mathrm{Na}^{22}$ source present, curve $B$ in its absence, and I denotes the point of intersection (1.5 Mev). The counting rate is normalized to 20 seconds. Since many such curves were taken throughout the investigation no special significance is to be attached to the numerical values of figure 11 .

To check on the stability of the electronic equipment throughout the continuous runs, a daily calibration procedure was adopted utilizing four $\mathrm{Na}^{22}$ sources which were attached to a fixed position near each detector. Any drift in the overall gain of the individual channels was determined by a daily monitor on the counting rate of some selected low attenuation helipot setting (such as at $C$ in figure 1l)。 For all the considered data the drift in counting rate never exceeded 
FIGURE 11

Energy Discrimination Curve 


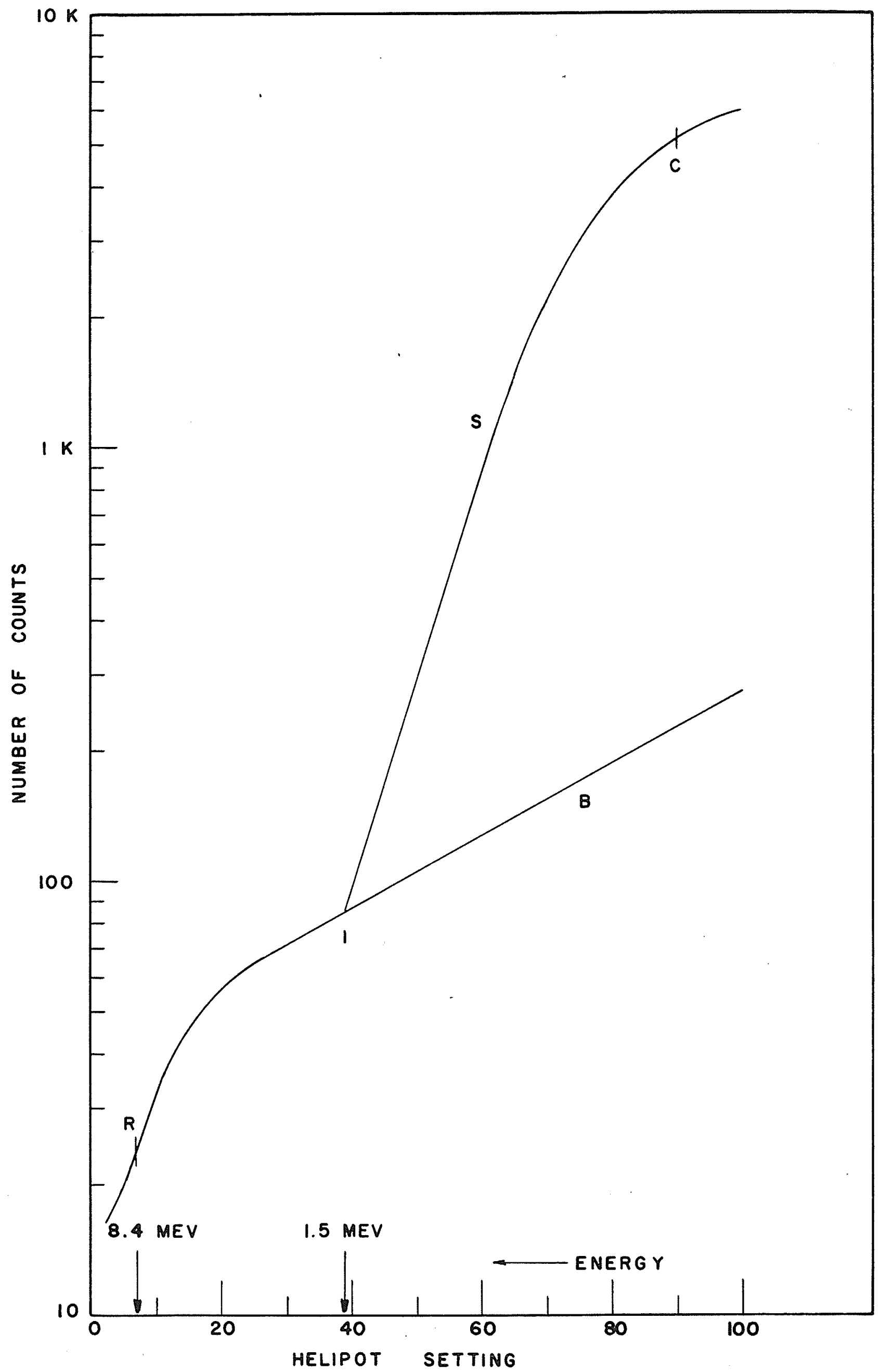


more than about $\pm 3 \%$ and for the major portion drift was less than 1\%. In the daily calibrations the decay of the sources was taken into account.

Stability checks on the coincidence circuits were made daily in a similar manner. With the helipot dials at $C$ for both coincidence detectors, the random coincidences in a fixed time interval between the two high single channel counting rates were measured. Again, allowing for the decay of the source, the random coincidence remained constant within statistical error.

The daily calibrations were taken at staggered times around the clock for two reasons. First, this removed any concern for a preferred 'drift time' during the day, and second, as the calibrations took approximately twenty-five minutes to complete, this staggering of the time of calibration meant that the burden of lost counting time was shared by all the times of day. The lost time was compensated for by normalization using previously collected data during that time interval as a standard. The telescopes, however, were kept rotating all the while the calibrations were being taken.

The proper functioning of the anticoincidence circuitry was periodically checked by means of a 60 cycle signal generator. The spectrum pulses from the detectors were removed and common pulses from the generator were simultaneously fed into the single channels of one coincidence system. These 
artificial coincidences were observed to be immediately cancelled when the same generator pulses were also fed to a third channel. The pulser was also used to check the performance of the four-fold coincidences.

The continuous monitoring of the cosmic radiation thus consisted of collecting data during the run at high pulse attenuation and calibrating at low attenuation.

\subsection{EXPERINENTATION}

The constant monitoring of the $\mu-$ meson component $>\sim 40$ Bev at Winnipeg, Manitoba may be readily classified into three time intervals. The first interval was from March 1 , 1963 to November 1, 1963. Data for this period were obtained with the narrow angle telescope as described in section 3.2. During this time the telescopes constantly sampled radiation from four viewing directions which remained constant with respect to the PES Iine, the plane of the telescopes being physically in the plane of the celestial equator. The four directions relative to the PES line observed during this long term run were $107.5^{\circ} \mathrm{E}, 17.5^{\circ} \mathrm{E}, 72.5^{\circ} \mathrm{W}$ and $162.5^{\circ} \mathrm{W}$.

In order to obtain data from a variety of viewing directions relative to the PES line the following procedure was then adopted: Data were accumulated for a period of several days from four viewing directions (since each telescope defines two directions with respect to the PES line) after which time the telescope motor was turned off for a fixed 
period of time. This stopping of telescope rotation was equivalent to an eastward shift of the viewing directions relative to the PES line. The eastward shift was $15^{\circ}$ per solar hour that the motor was inoperative. Thus, after the rotation was resumed, the telescopes sampled radiation from four new directions, each shifted eastward from their previous values.

The second time interval was from November 1,1963 to December 25, 1963 during which time the entire celestial equator was traced out for a variety of viewing directions. During this time sweeps of the spectrum of viewing directions were made utilizing the narrow angle telescopes.

The third time interval included all the data accumulated after December 25, 1963, during which time the wide angle geometry was employed. During this period data from four viewing directions were accumulated for a period of two days, after which time the rotation of the polar axis was stopped for exactly two hours. Upon resumption of rotation the viewing directions were shifted $30^{\circ}$ east of their former values. Then data were collected from these new directions for another two day period until once again the rotation was stopped for two hours and so on. In this manner the celestial equator was scanned in $30^{\circ}$ intervals, completing one full scan in a little over six days. Usually, however, the scans took seven days to complete in order to avoid the necessity of 
returning to the laboratory for two hours in the middle of the night.

The use of the wide angle telescopes suffered the disadvantage of poorer angular resolution (the coincidence solid angle was increased by a factor of $w 3$ over the narrow angle), however, the sacrifice in angular resolution was compensated for by greatly improved statistics. The better statistics enabled shorter observation times for any particular direction relative to the $\mathrm{PES}$ line, and this in turn enabled many more sweeps of the celestial equator to be made in the same time interval.

In all experimental runs the equipment stability and performance were checked daily. 
$\underline{P A R T} \quad \underline{F} \mathrm{O} R$

DISCUSSION OF OBSERVATIONS 
4.1 EXPERIMENTAL RESULTS

The data presented in this thesis have been obtained during the present 'quiet' part of the solar cycle (March, 1963-July, 1964). They are the results of preliminary investigations using the two geometrically identical $\mu-m e s o n$ telescopes in the manner described in the last section. Some of these results have been presented at the C.A.P. and A.P.S. Conferences (Standil and Bukata, 1964a, 1964b).

Figure 12 illustrates the PES line showing the four directions observed during the long-term narrow angle run in the interval March 1 to November 1, 1963. During this run these four directions $\left(17.5^{\circ} \mathrm{E}, 107.5^{\circ} \mathrm{E}, 72.5^{\circ} \mathrm{W}\right.$, and $\left.162.5^{\circ} \mathrm{W}\right)$ were kept fixed with respect to the PES line, but not with respect to location on the celestial equator. Due to the difference between solar and sidereal times all four telescope extremities gradually traced out the celestial equator in an easterly direction. Table 4.1 gives the hour circle location (RA) of each telescope extremity as a function of time during the long term run. These tabulated values locate each telescope's position in ten day intervals beginning March 1 and were determined by reference to the SCI Constellation Chart of figure 2 . 
FIGURE 12

Viewing Directions Relative to the PES Iine During Narrow Angle Run 


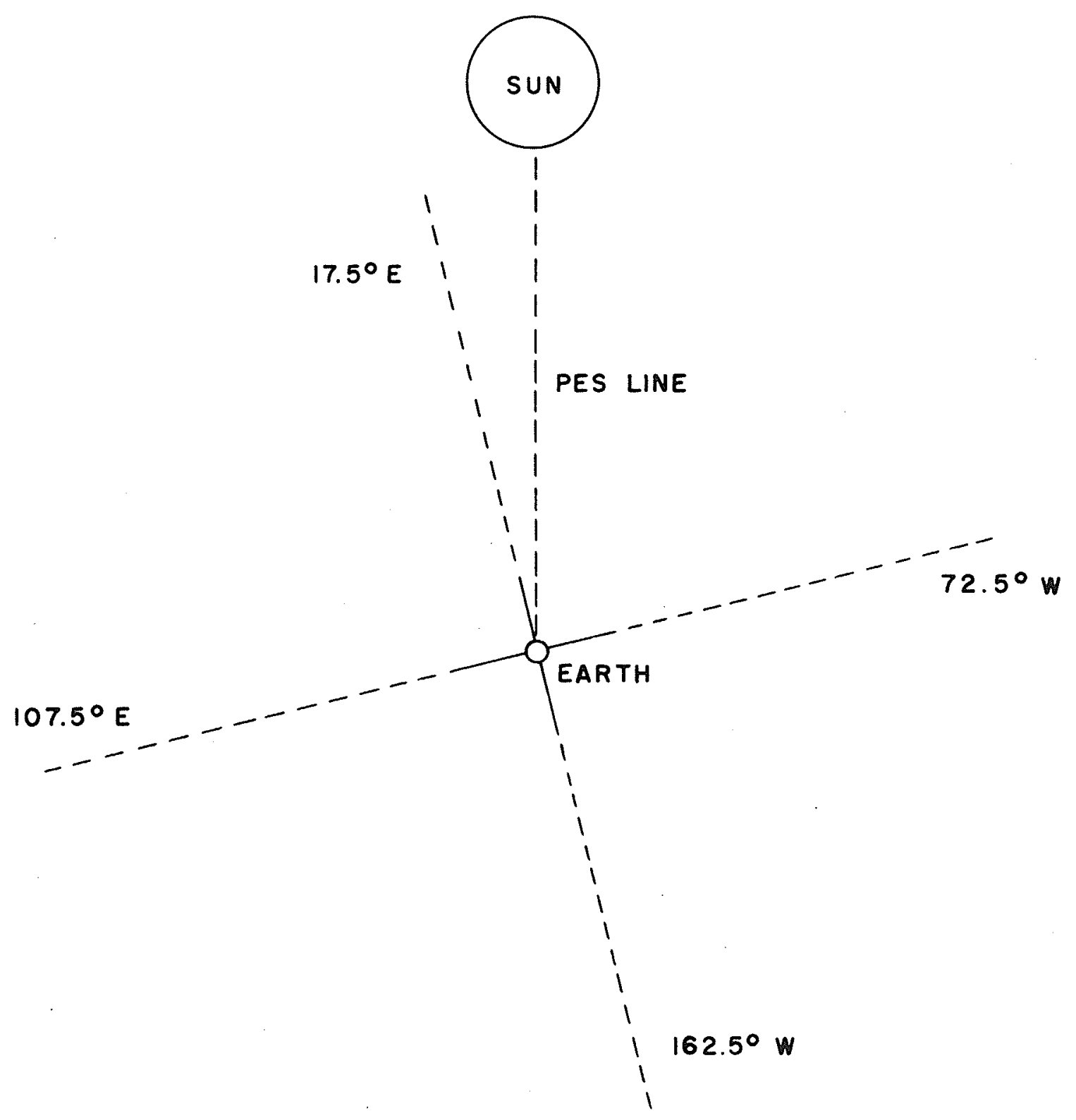


TABLE 4.1

Hour Circle Locations of the Four Viewing

Directions During Long Term Narrow Angle Run

\begin{tabular}{|c|c|c|c|c|}
\hline \multirow[b]{2}{*}{ Date } & \multicolumn{4}{|c|}{ Direction } \\
\hline & $17.5^{\circ} \mathrm{E}$ & $107.5^{\circ} \mathrm{E}$ & $72.5^{\circ} \mathrm{W}$ & $162.5^{\circ} \mathrm{WV}$ \\
\hline Mar. 1 & $24.0 h$ & $6.0 \mathrm{~h}$ & $18.0 \mathrm{~h}$ & $12.0 \mathrm{~h}$ \\
\hline 11 & 0.6 & 6.6 & 18.6 & 12.6 \\
\hline 21 & 1.2 & 7.2 & 19.2 & 13.2 \\
\hline 31 & 1.8 & 7.8 & 19.8 & 13.8 \\
\hline Apr.10 & 2.4 & 8.4 & 20.4 & $14 \cdot 4$ \\
\hline 20 & 3.0 & 9.1 & 21.1 & 15.1 \\
\hline 30 & 3.7 & 9.7 & 21.7 & 15.7 \\
\hline May 10 & $4 \cdot 3$ & 10.3 & 22.3 & 16.3 \\
\hline 20 & 5.0 & 11.0 & 23.0 & 17.0 \\
\hline 30 & 5.6 & 11.6 & 23.6 & 17.6 \\
\hline Jun. 9 & 6.3 & 12.3 & 0.3 & 18.3 \\
\hline 19 & 7.0 & 13.0 & 1.0 & 19.0 \\
\hline 29 & 7.7 & 13.7 & 1.7 & 19.7 \\
\hline JuI. 9 & 8.4 & $14 \cdot 4$ & 2.4 & 20.4 \\
\hline 19 & 9.1 & 15.1 & 3.1 & 21.1 \\
\hline 29 & 9.8 & 15.8 & 3.8 & 21.8 \\
\hline Aug. 8 & 10.4 & 16.4 & $4 \cdot 4$ & 22.4 \\
\hline 18 & 11.0 & 17.0 & 5.0 & 23.0 \\
\hline 28 & 11.6 & 17.6 & 5.6 & 23.6 \\
\hline
\end{tabular}


TABLE 4.1 (cont.)

\begin{tabular}{rrrrr} 
Date & $17.5^{\circ} \mathrm{E}$ & $107.5^{\circ} \mathrm{E}$ & $72.5^{\circ} \mathrm{W}$ & $162.5^{\circ} \mathrm{W}$ \\
\hline Sept.7 & 12.2 & 18.2 & 6.2 & 0.2 \\
17 & 12.8 & 18.8 & 6.8 & 0.8 \\
27 & 13.4 & 19.4 & 7.4 & 1.4 \\
Oct. 7 & 14.0 & 20.0 & 8.0 & 2.0 \\
17 & 14.7 & 20.7 & 8.7 & 2.7 \\
27 & 15.3 & 21.3 & 9.3 & 3.3 \\
Nov. 6 & 15.9 & 21.9 & 9.9 & 3.9 \\
16 & 16.6 & 22.6 & 10.6 & 4.6 \\
26 & 17.3 & 23.3 & 11.3 & 5.3 \\
Dec. 6 & 18.0 & 0.0 & 12.0 & 6.0 \\
16 & 18.7 & 0.7 & 12.7 & 6.7 \\
26 & 19.5 & 1.5 & 13.5 & 7.5 \\
Jan. 5 & 20.2 & 2.2 & 14.2 & 8.2 \\
15 & 21.0 & 3.0 & 15.0 & 9.0 \\
25 & 21.7 & 3.7 & 15.7 & 9.7 \\
Feb. 4 & 22.4 & 4.4 & 16.4 & 10.4 \\
14 & 23.1 & 5.1 & 17.1 & 11.1 \\
24 & 23.6 & 5.6 & 17.6 & 11.6
\end{tabular}

Figure 13 shows the integrated results of this 8 -month narrow angle run. During this run 221 days of useful data were obtained, and these data were normalized to weekly 
FIGURE 13

Bi-Weekly Totals of Narrow Angle Run as a Function of Celestial Equator Location 


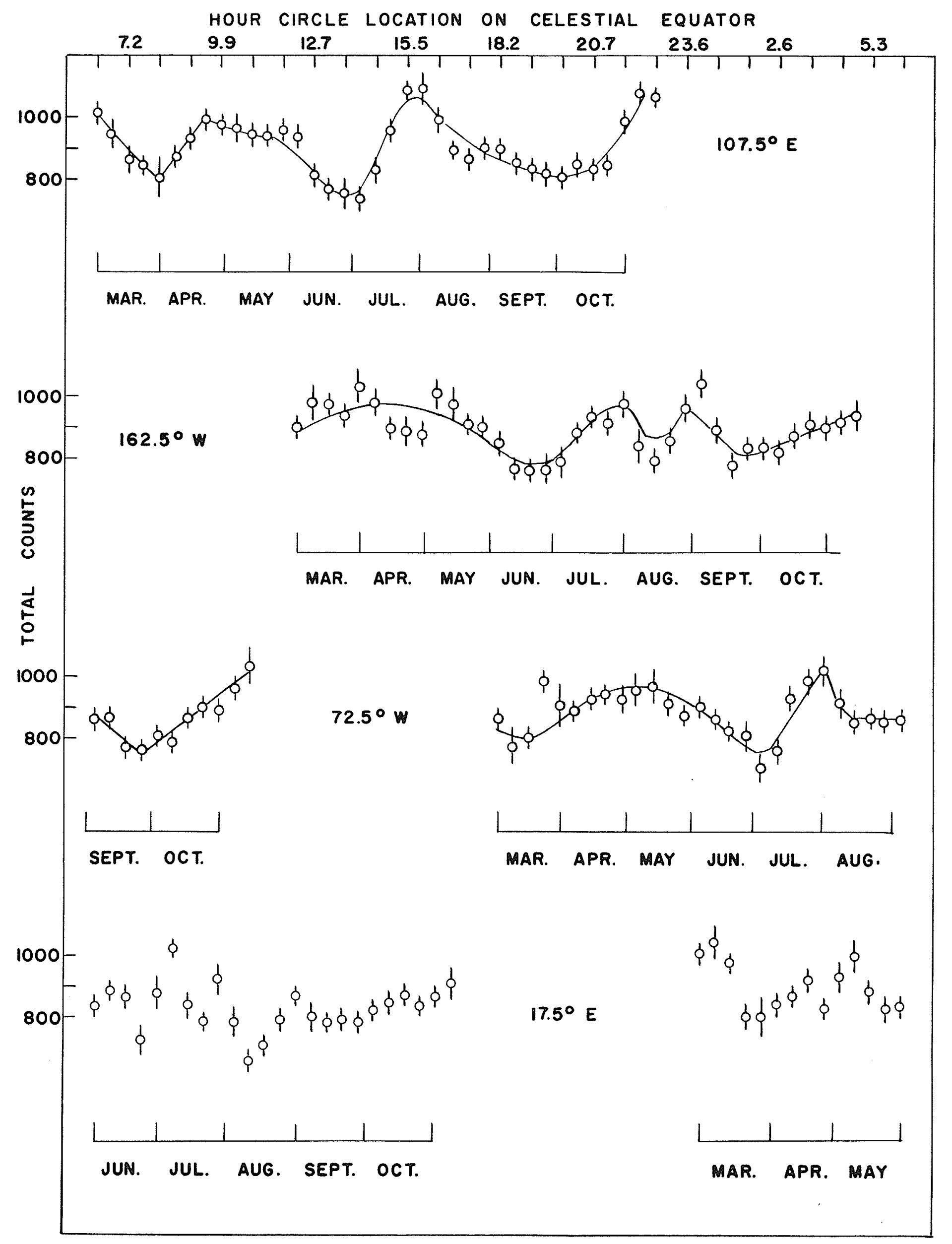


totals for each of the four viewing directions. In order to compensate for the low counting rates, the results in figure 13 have been plotted as overlapping bi-weekly totals.

Due to the $90^{\circ}$ separation of each of the viewing directions, one quarter of a year after one telescope had occupied an orientation on the celestial equator the second telescope occupied the same orientation. Thus each successive curve in figure 13 has been displaced 91 days from the preceding curve so as to correspond to the same hour circle location on the celestial equator. Smooth curves have been drawn through the plotted points.

Intensity variations of amplitudes as high as about $\pm 12 \%$ are apparent on at least three of the curves (the curves for $107.5^{\circ} \mathrm{E}, 162.5^{\circ} \mathrm{W}$, and $72.5^{\circ} \mathrm{W}$ ) while the curve for $17.5^{\circ} \mathrm{E}$ shows somewhat more statistical scatter. Some striking consistencies are evident in figure 13. The curve for $107.5^{\circ} \mathrm{E}$ shows a pronounced maximum between $14 \mathrm{~h}$ and $17 \mathrm{~h}$ while $162.50 \mathrm{~W}$ indicates a much broader peak between $12 \mathrm{~h}$ and 18h. Each of the directions $107.5^{\circ} \mathrm{E}, 162.5^{\circ} \mathrm{W}$ and $72.5^{\circ} \mathrm{W}$ indicate enhanced counting rates between about $20 \mathrm{~h}$ and $2 \mathrm{~h}$. Both the directions $107.5^{\circ} \mathrm{E}$ and $72.5^{\circ} \mathrm{W}$ indicate increased counting rates between $9 \mathrm{~h}$ and $\mathrm{Ilh}$ and possible maxima around $6 \mathrm{~h}$. In addition $162.5 \mathrm{~W}$ displays a possible peak approaching $6 \mathrm{~h}$, while $72.5^{\circ} \mathrm{W}$ has a peak near 4h. The Milky way intersects the galactic equator in two regions, between $18 \mathrm{~h}$ and $19.5 \mathrm{~h}$ and between $6.5 \mathrm{~h}$ and 
7.5h. The enhanced counting rates therefore appear to occur not from the directions of the central regions of the galaxy but rather a few hour circles eastward. In fact $8 \mathrm{~h}$ appears to be a region of reduced intensity just eastward of the Milky Way. Other consistent minima appear to be at about $2 \mathrm{~h}$ and $19.5 \mathrm{~h}$, the former being the location of the shorter less dense galactic arm and the latter again just eastward of the Milky Way. In addition the telescope $107.5^{\circ}$ E observed a minimum counting rate from $13 \mathrm{~h}$, again a short sparsely occupied arm of the galaxy. Figure 13 therefore suggests preferred arrival directions of cosmic radiation from galactic space. The preferred viewing directions appear to be defined by RA about $10 \mathrm{~h}, 16 \mathrm{~h}, 2 \mathrm{I}-23 \mathrm{~h}$, and $4-6 \mathrm{~h}$.

It is also noted that the telescope constantly trained $17.5^{\circ}$ east of the sun displayed an intensity variation which was considerably less regular than the other three. More statistical fluctuation is evident although resolution of peaks and valleys may be present to some extent.

As mentioned in Section 2 and considered in Appendix II each telescope in the course of its daily rotation goes through a reflected sequence of zenith angle orientations between $\phi=90^{\circ}$ (when the telescope is in the horizontal plane) and $\phi=49.9^{\circ}$ (when the telescope is in the vertical plane). On the basis of a $\operatorname{Cos}^{n} \phi$ intensity law, the detectors will record low counting rates at large zenith angles and 
higher counting rates at smaller zenith angles. This explains the nature of the histograms depicted in figure 140 These histograms give the time of arrival distributions as observed for each of the four viewing directions during the first 162 days ( 6 months) of the narrow angle run. The data were analyzed in 20-minute intervals. The dashed curves drawn are the distributions that would be expected in the present work assuming an intensity-zenith angle variation of the form $j(\phi)=5.2 \operatorname{Cos}^{1.25} \phi$. (The basis of this relation will be discussed later.) There is good agreement between the observed and the expected distributions for three of the four viewing directions. However, for the viewing direction $17.5^{\circ}$ east of the PES line there is a pronounced peak shift to a time about 50 minutes earlier than would be expected on the basis of this $\cos ^{n} \phi$ law. The theoretically expected time of maximum intensity was 13:40 LST (time at which the telescope was in the vertical plane with $\left.\phi=49.9^{\circ}\right)$. The observed time of maximum intensity was about 12:50 LST.

The histograms for the full 221 day run were essentially similar to those shown in figure 14 but are not included as the two telescopes were interchanged after 162 days. After 6 months of continuous running it became statistically conclusive that the viewing direction $17.5^{\circ} \mathrm{E}$ was consistently showing a peak shift. To eliminate the possibility that this was merely a property of the observing telescope and not due 
81

FIGURE 14

Time of Arroival Distributions for the Four Viewing Directions During the First 162 Days of the Narrow Angle Run 


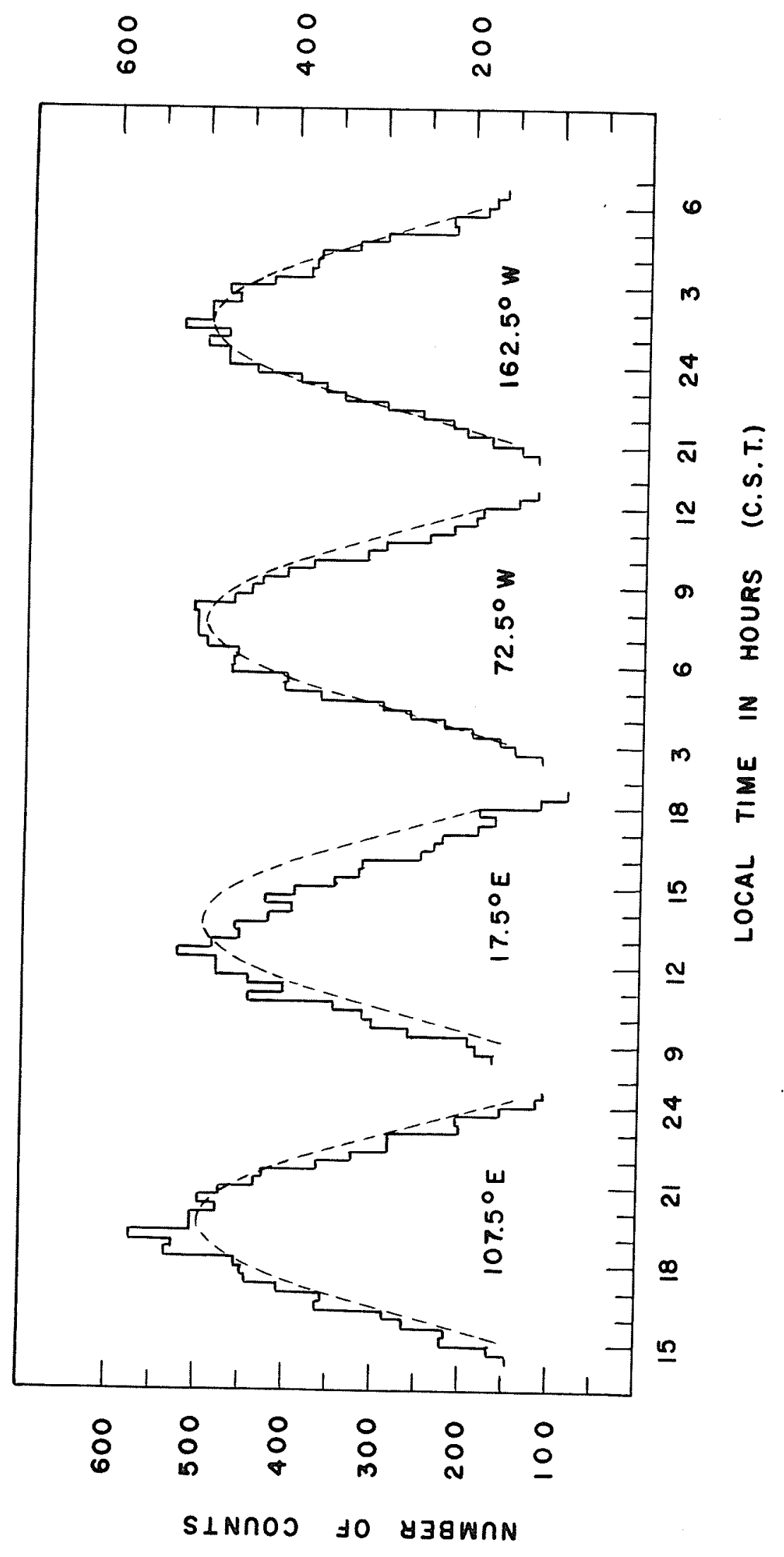


to the particular viewing direction, the telescopes were interchanged for the last two months of the run. The telescope which initially observed $17.5^{\circ} \mathrm{E}$ and $162.5^{\circ}$ was now set to observe $72.5^{\circ} \mathrm{W}$ and $107.5^{\circ} \mathrm{E}$, and vice-versa. This was accomplished by merely stopping the rotation for exactly 6 hours thereby shifting each telescope eastward by $90^{\circ}$. This interchanging of telescopes did not change the nature of the observations. The viewing direction $17.5^{\circ} \mathrm{E}$ continued to display the shift to an earlier time, while no significant peak shifts appeared for the other three directions. This is illustrated in figure 15 in which the time of arrival histograms for $17.5^{\circ} \mathrm{E}$ are presented for each month of the long term run. The histograms for September and October, which also display the peak shifts were obtained after the telescopes had been interchanged. Here again the superimposed dashed curves represent the expected distributions assuming $j(\phi)=5.2 \cos ^{1.25} \phi$. The reason for the varying sizes of the comparison functions is simply that not every month contained the same number of days of useful counting time. By way of comparison similar monthly histograms for the viewing direction $162.5^{\circ}$ are presented in figure 16. It is evident that no such large peak shifts are prominent. Curves (not shown) similar to figure 16 were also obtained for the viewing directions $107.5^{\circ} \mathrm{E}$ and $72.5^{\circ} \mathrm{W}$. The eastward shift (towards an earlier local solar time) 


\section{FIGURE I5}

Monthly Time of Arrival Distributions for Viewing Direction $17.5^{\circ} \mathrm{E}$ of the PES Line 


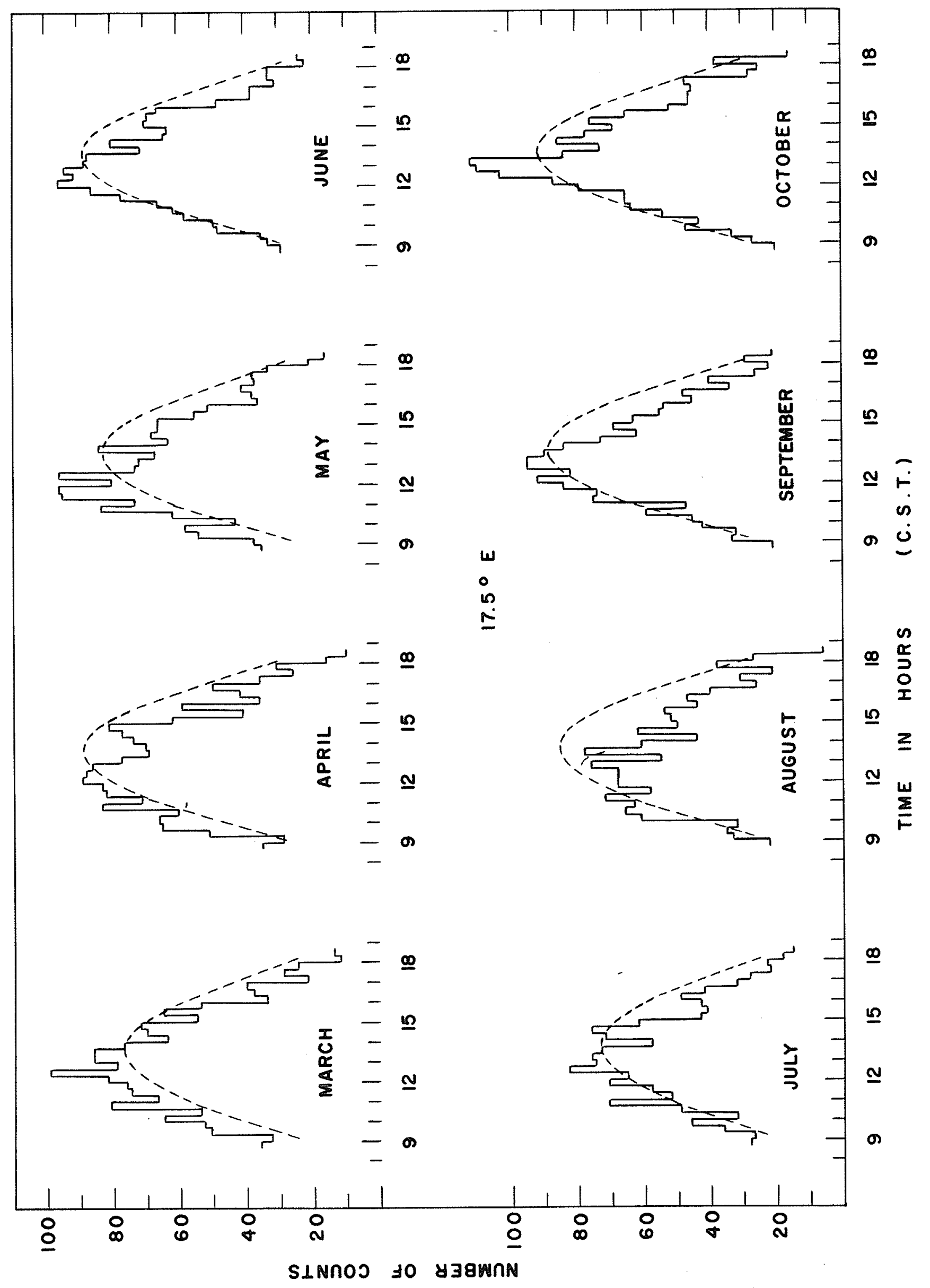


FIGURE 16

Monthly Time of Arrival Distributions for Viewing Direction $162.50 \mathrm{~W}$ of the PES Line 


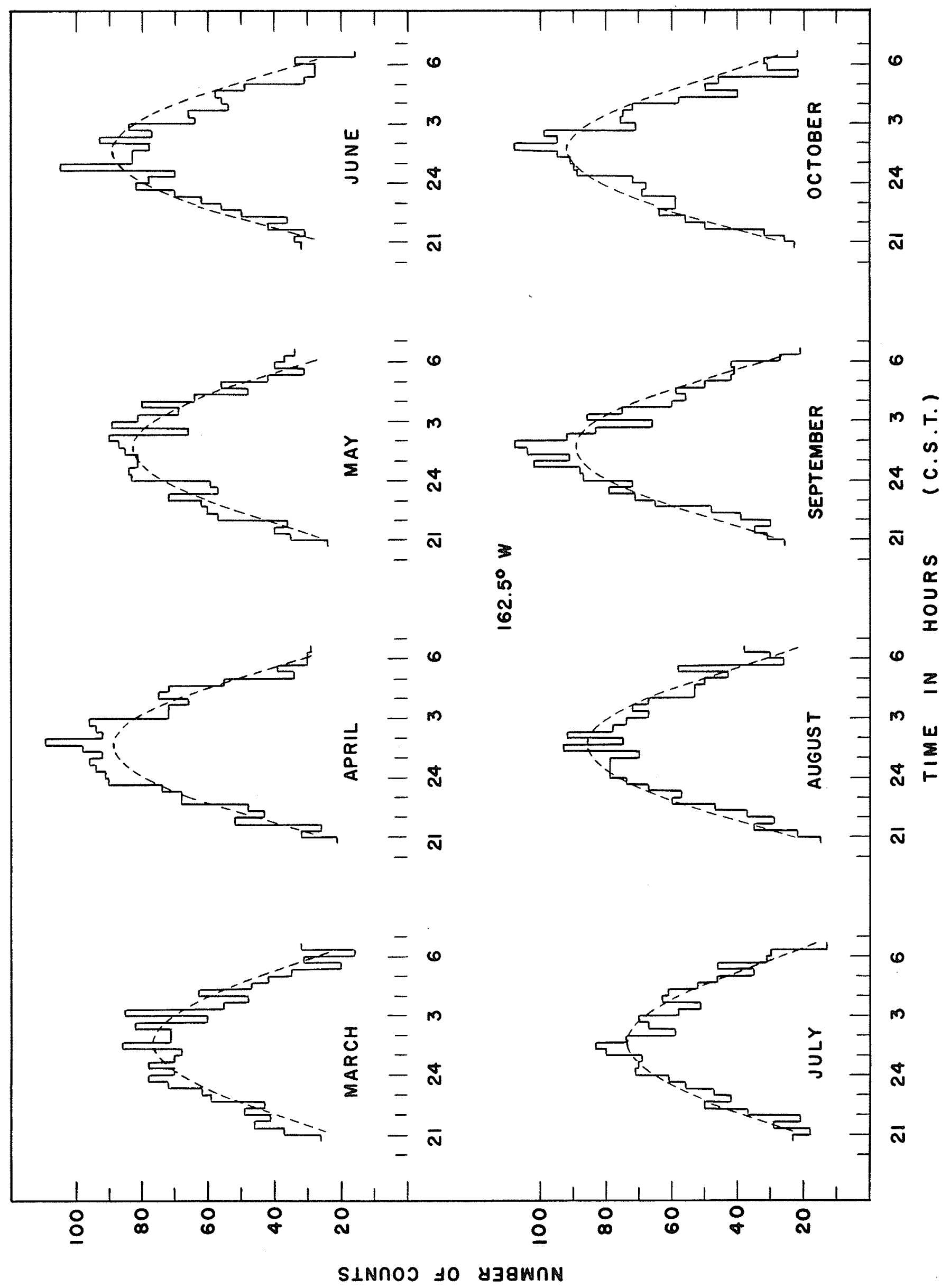


of the peak of the histogram distributions for the viewing direction $17.5^{\circ} \mathrm{E}$ is consistent with more radiation impinging upon the earth from a direction to the west of the viewing direction of the telescope than to the east. This differential in intensity may be brought about either by a reduction of intensity from the east or by a beam of radiation more intense than the steady background stream approaching the earth from a direction west of $17.5^{\circ} \mathrm{E}$ of the PES line. This may be seen on the basis of a rotating atmosphere picture which is illustrated in figure 17. The telescope samples radiation from a fixed spatial direction (relative to the PES line) while rotating from east to west through an essentially stationary (with respect to the earth) atmosphere. Equivalently, the telescope may be considered to be at rest and the atmosphere taken to be rotating about the telescope from west to east. This atmospheric rotation is shown in figure 17 where $A^{\prime}$ ' represents the solid angle of the telescope directed $17.5^{\circ} \mathrm{E}$ of the PES line. The absorbent atmosphere is shown rotating about this telescope, $\mathrm{XZ}$ representing the atmosphere at 12:40 LST, X'Z' at 13:40 LST and $X^{\prime \prime}$ at 14:40 LST. A beam B of radiation is shown approaching the telescope from a direction west of the telescope's viewing direction. The height above the detector of the average meson production level $(\sim 100 \mathrm{mb})$ is shown at $C, D$ and $E$ for $12: 40,13: 40$ and $14: 40$ LST respectively. This increasing 
FIGURE 17

Rotating Atmosphere Picture 
height of the meson production level coupled with the corresponding increase in atmospheric absorption with increasing time results in a peak shift towards earlier time in the laboratory frame of reference.

Figure 17 considers increased intensity from a direction west of $17.5^{\circ} \mathrm{E}$ as producing the differential between directions east and west of $17.5^{\circ} \mathrm{E}$ of the PES line. The same effect may be caused by a reduction in intensity of the radiation impinging upon the detector from a direction east of the telescope's viewing direction. In fact, this premise is consistent with the results of the 8 month run where the area under the histogram for $17.5^{\circ} \mathrm{E}$ was about $(5 \pm 2) \%$ less than the areas under the histograms for the other three viewing directions. Table 4.2 shows the total number of events recorded during the long term run (area under a histogram for a 10 hour interval centred on the time of expected maximum) for the four viewing directions considered.

\section{TABLE $4 \cdot 2$}

TOTAL NUMBER OF EVENTS RECORDED FROM MARCH 1 TO NOVEMBER I, 1963

Viewing Direction Relative to the PES line

$$
\begin{array}{r}
72.5^{\circ} \mathrm{W} \\
162.55^{\circ} \mathrm{W} \\
107.5^{\circ} \mathrm{E} \\
17.5^{\circ} \mathrm{E}
\end{array}
$$

Number of Events Summed over Ten Hour Intervals

$13,832 \pm 117$
$14,109 \pm 119$
$14,064 \pm 118$
$13,328 \pm 115$


The peak shifts observed for the viewing direction $17.5^{\circ} \mathrm{E}$ represent a departure from the $\cos ^{n} \emptyset$ zenith variation law. This departure occurs during a small change in zenith orientation. The eastward peak shift with reduced intensity is about 50 minutes, during which time the zenith angle changes by only about $1.6^{\circ}$ from $51.5^{\circ}$ to $49.9^{\circ}$.

The 'garden-hose' model of the solar magnetic field (Parker, 1958, 1963: IG Bulletin, 1964) is illustrated in figure It showing the interplanetary magnetic field lines in an Archimedes spiral configuration. Such a field in conjunction with scattering in a solar atmosphere may present a mechanism which affects intergalactic cosmic ray particles approaching the earth from the vicinity of the sun, this mechanism being responsible for the peak shifts and reduced intensity observed for the viewing direction $17.5^{\circ} \mathrm{E}$. The nature of such a solar mechanism is somewhat speculative. However, a possible mechanism for explaining a reduction in intensity for viewing directions just east of the PES Iine is as follows.

There may exist a solar effect similar in nature to the geomagnetic latitude effect observed due to the earthis field. 
FIGURE 18

The 'Garden Hose: Model of the Solar Magnetic Field 


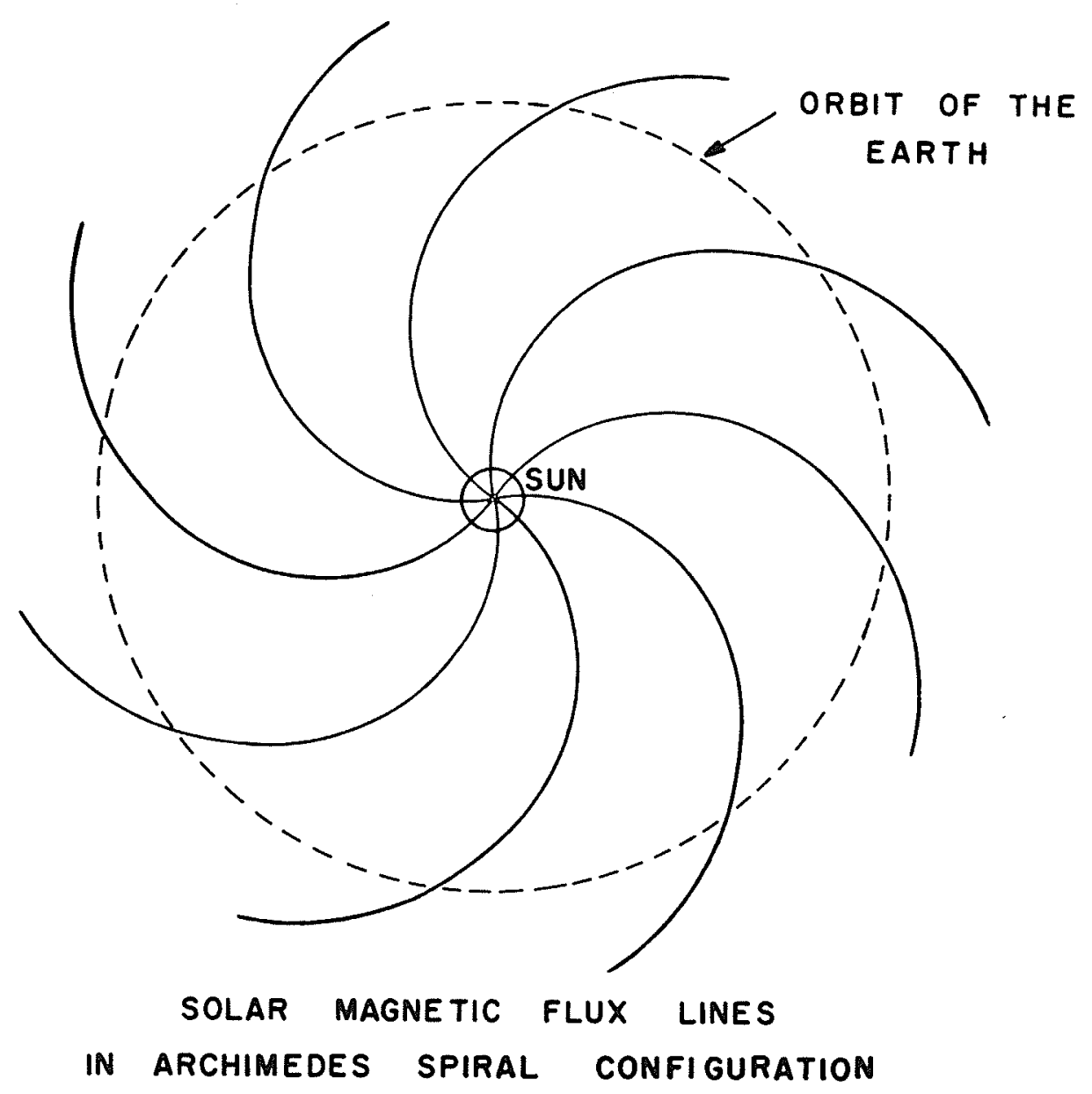


Due to the dipole nature of the earth's field, the critical cut off energy for impinging primaries is greatest near the equator $(\sim 20 \mathrm{Bev})$ and gradually decreases to nearly zero at the poles. Thus at the equator all that part of the primary radiation below about $20 \mathrm{Bev}$ fails to reach the top of the atmosphere thereby causing equatorial regions to observe lower total intensities than do regions of higher latitudes. A similar condition may exist in which the solar field introduces a cut-off energy for particles approaching the earth. This cut-off energy would have to be a function of PES direction, being higher on the east of the PES line than on the west of the PES Iine. This would mean that a telescope monitoring radiation from, say, $17.5^{\circ}$ east of the PES line would be sampling radiation above some energy $I_{1}$ while a telescope pointing $17.5^{\circ}$ west of the PES line would be sampling radiation above an energy $E_{2}$ where $E_{2}<E_{1}$.

Satellite measurements of the solar field now planned and in progress will enable theoretical calculations as a check of the above idea.

It might be argued that since the long term narrow angle 
run did not continue for a full year, but rather fell 4 months short, the decrease in total counting rate observed from $17.5^{\circ} \mathrm{E}$ was due to the fact that each telescope traced out a different two-thirds of the celestial equator. This argument may even be emphasized by the fact that the telescope pointing $17.5^{\circ} \mathrm{E}$ passed through the Milky Way region but one and, in fact, did not have the opportunity to scan the region 20-23h (region of enhanced counting rates for the other three viewing directions and also the region which appears to be most intense during the wide angle runs to be soon discussed). However, it is evident from the appearance of the time-variation curve for $17.5^{\circ}$ (figure 13 ) the scatter in counting rate is greater than that for the other three directions farther removed from the PES Iine and an attempt to fit a smooth curve to the scattered points is more difficult. The peaks and valleys are harder to discern and do not indicate the same 'source' locations as do the other three viewing directions. As an example of this, it is noted that the region of the celestial equator around $\mathrm{RA} \sim 10 \mathrm{~h}$ which shows definite peaks from $107.5^{\circ} \mathrm{E}$ and $72.5^{\circ} \mathrm{W}$ is represented by a valley when observed from the viewing direction $17.5^{\circ} \mathrm{E}$. This is taken as further evidence of a solar modulation effect.

The short-term wide angle runs were treated in a similar manner to the long-term narrow angle run, but contained data from an additional number of viewing directions. This thesis 
includes data collected during 28 complete sweeps of the celestial equator via $30^{\circ}$ increments. The results of the first eight scans is shown in figure 19 as time-of-arrival distributions similar to the histograms of figure 14 but for the increased number of viewing directions. Each histogram is the result of 16 days of counting except those at $45^{\circ} \mathrm{E}, 135^{\circ} \mathrm{W}, 45^{\circ} \mathrm{W}$, and $135^{\circ} \mathrm{E}$ where additional data have been obtained. Counting time at $45^{\circ} \mathrm{E}$ and $135^{\circ} \mathrm{W}$ was 25 days and at $45^{\circ} \mathrm{W}$ and $135^{\circ} \mathrm{E}$ was 24 days. The analysis was again performed on a 20-minute basis. However the dashed curves in this case represent the distributions that would be expected on the basis of an intensity-zenith angle law $j(\phi)=20.0 \cos ^{1.60} \phi$ (to be discussed later). With the poorer angular resolution any peak shifts are certainly considerably reduced. The histogram for $1.5^{\circ} \mathrm{E}$ still shows evidence of a tendency to shift to an earlier hour.

These data were collected between December 25 and February 29 and their outstanding feature is displayed in figure 20 which shows the integrated peak area (area under the experimental histograms) as a function of viewing direction. A striking change is evident as the viewing direction changes from $15^{\circ} \mathrm{E}$ to $15^{\circ} \mathrm{W}$ of the PES line. The large change observed as the viewing direction crosses the PES line indicates an anisotropy $\frac{(I \max -I \min )}{I \max +I \min )} \times 100 \%$ of about $11 \%$ which is considerably higher than anisotropies observed by other 
FIGURE 19

Time of Arrival Distributions for Each Viewing Direction During the First Eight Wide Angle Scans of the Celestial Equator 


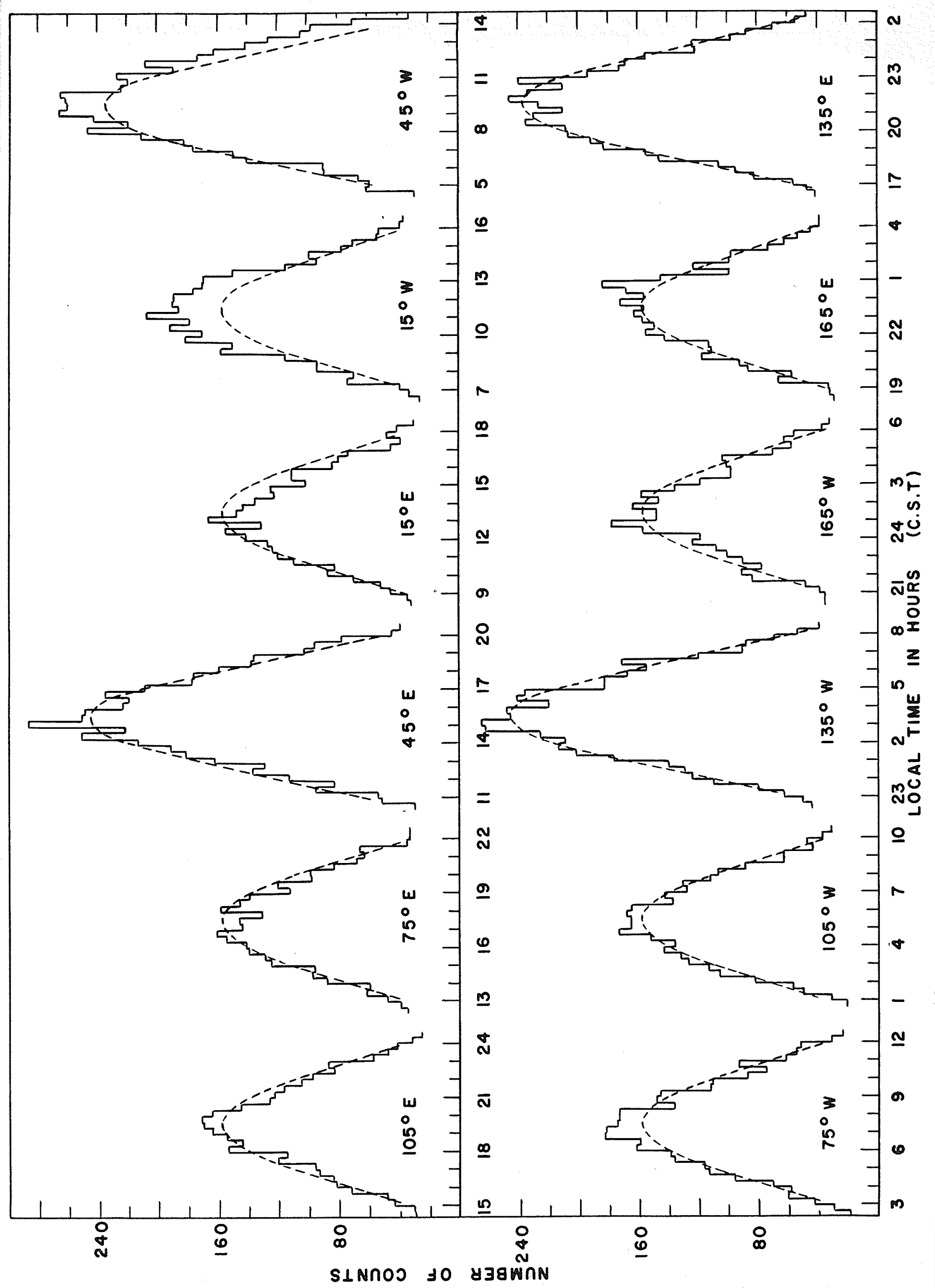


investigators using conventional neutron or meson monitors in their studies of diurnal variations. Diurnal amplitudes of about 0.1 - $1.0 \%$ have been reported by such workers as Jacklyn (1963), Rao et al (1963) and Fenton et al (1961). However, for the reasons outlined in Section 2 the results of the present investigation are not directly comparable with most of the previous work. The conventional detectors have different energy responses and are not so directionally selective. The closest simulations to the present experimental arrangement are fixed telescopes vertically mounted at equatorial stations. Such telescopes scan primaries arriving in or near the galactic equatorial plane. However, a comparison of their primary arrival direction asymptotic coordinates with those applicable to the present rotating telescopes shows a significant difference. For primary particles above $40 \mathrm{Bev}$ equatorial stations have a small spread in asymptotic latitudes $\left(\sim 5^{\circ}\right.$ or less) and a large spread in asymptotic longitudes ( $\sim 35^{\circ}$ or more). The appropriate sky windows for the telescopes located at Winnipeg are illustrated in figure 2l. Seven windows are shown corresponding to the zenith angle-azimuth angle orientations of a telescope every two hours. The succession of windows is consistent with the telescope's rotating in the laboratory system from an azimuth angle of due east to an azimuth angle of due west. The passage of local time is such that the 


\section{FIGURE 21}

Asymptotic Viewing Directions (Sky Windows) for Winnipeg, Manitoba

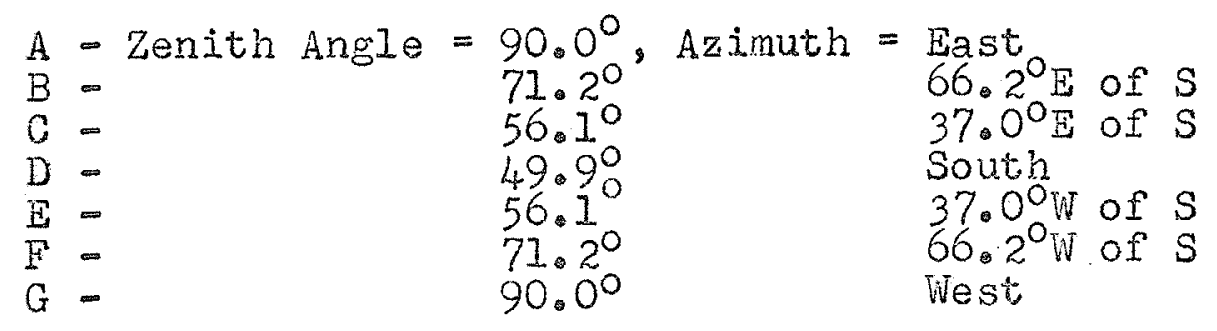

Calculations Supplied by Dr. M. Bercovitch, Chalk River Nuclear Laboratories 


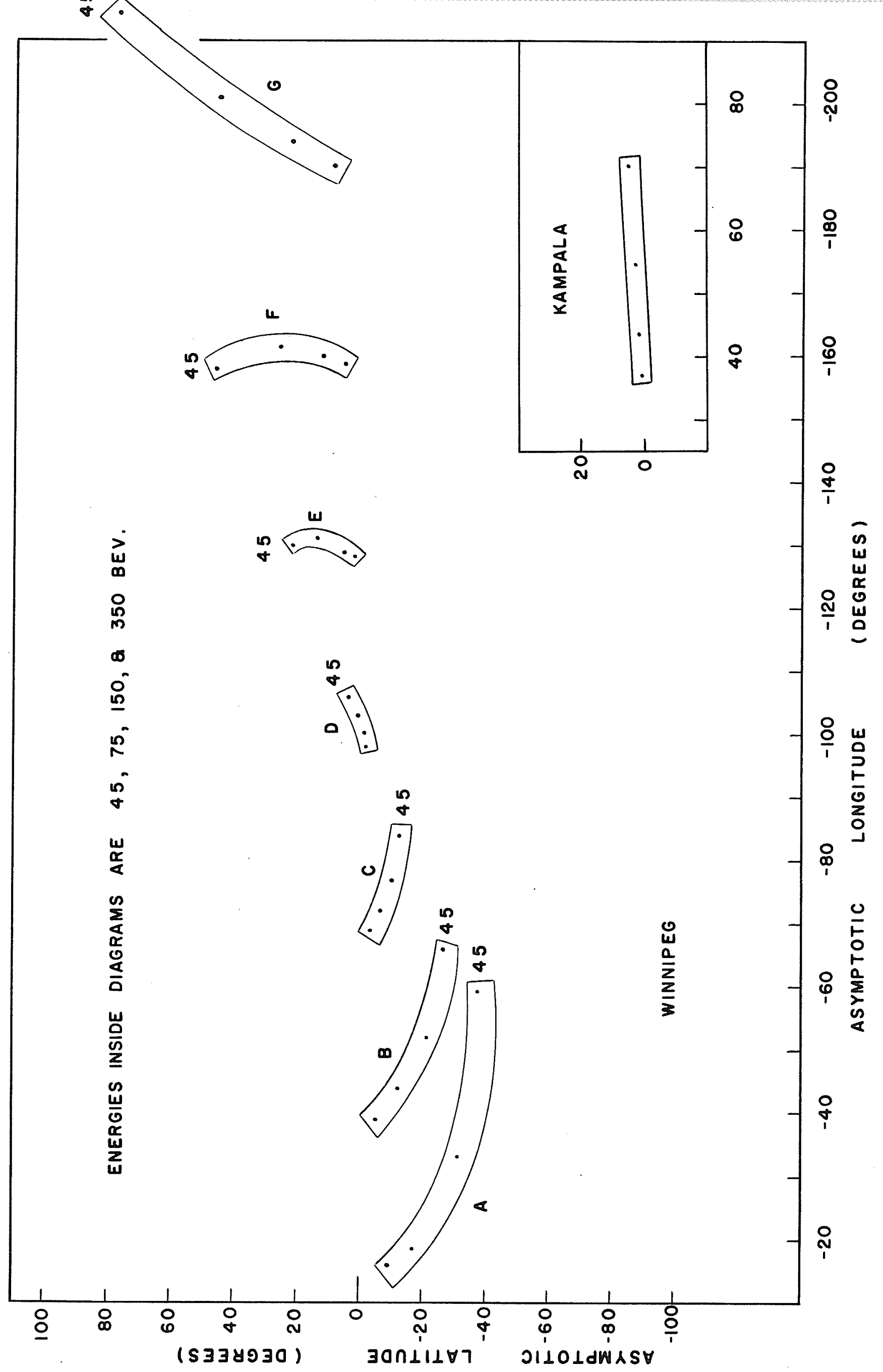


viewing orientations of the telescope with respect to the earth move from left to right in the figure. The zenith angle-azimuth angle orientations of the telescope with respect to the laboratory are given in the caption. It is seen that for a rotating telescope when the zenith angle is $49.9^{\circ}$, south of the vertical (the position of the telescope when it records its maximum counting rate), the corresponding asymptotic latitude and longitude spreads (for an energy range $45-350$ Bev) are about $5^{\circ}$ and $10^{\circ}$ respectively. Two hours earlier these are about $10^{\circ}$ and $15^{\circ}$ while two hours later they are about $22^{\circ}$ and $2^{\circ}$. For comparison the sky window (energy range 45 - $350 \mathrm{Bev}$ ) for a stationary telescope located at the equatorial station Kampala (geographic latitude $0.33^{\circ} \mathrm{N}$, geographic longitude $32.56^{\circ} \mathrm{E}$ ) is shown as an inset in figure 21. The effective average asymptotic acceptance cones for the present rotating telescopes are thus relatively much narrower in terms of asymptotic longitudes than the corresponding cones for equatorial stations. This fact enables the rotating telescopes at this location to sample radiation from directions well defined with respect to longitude.

The nature of the effects observed during the long term narrow angle run and the first eight wide angle scans have recently appeared in print (Standil and Bukata, 1964c).

It is interesting to note that between November $I$ and December 15, 1963 a similar scan was made of the celestial 


\section{FIGURE 22}

Variation of Observed Peak Area (in a total of 6 days) With Viewing Direction During Single Narrow Angle Scan of the Celestial Equator 


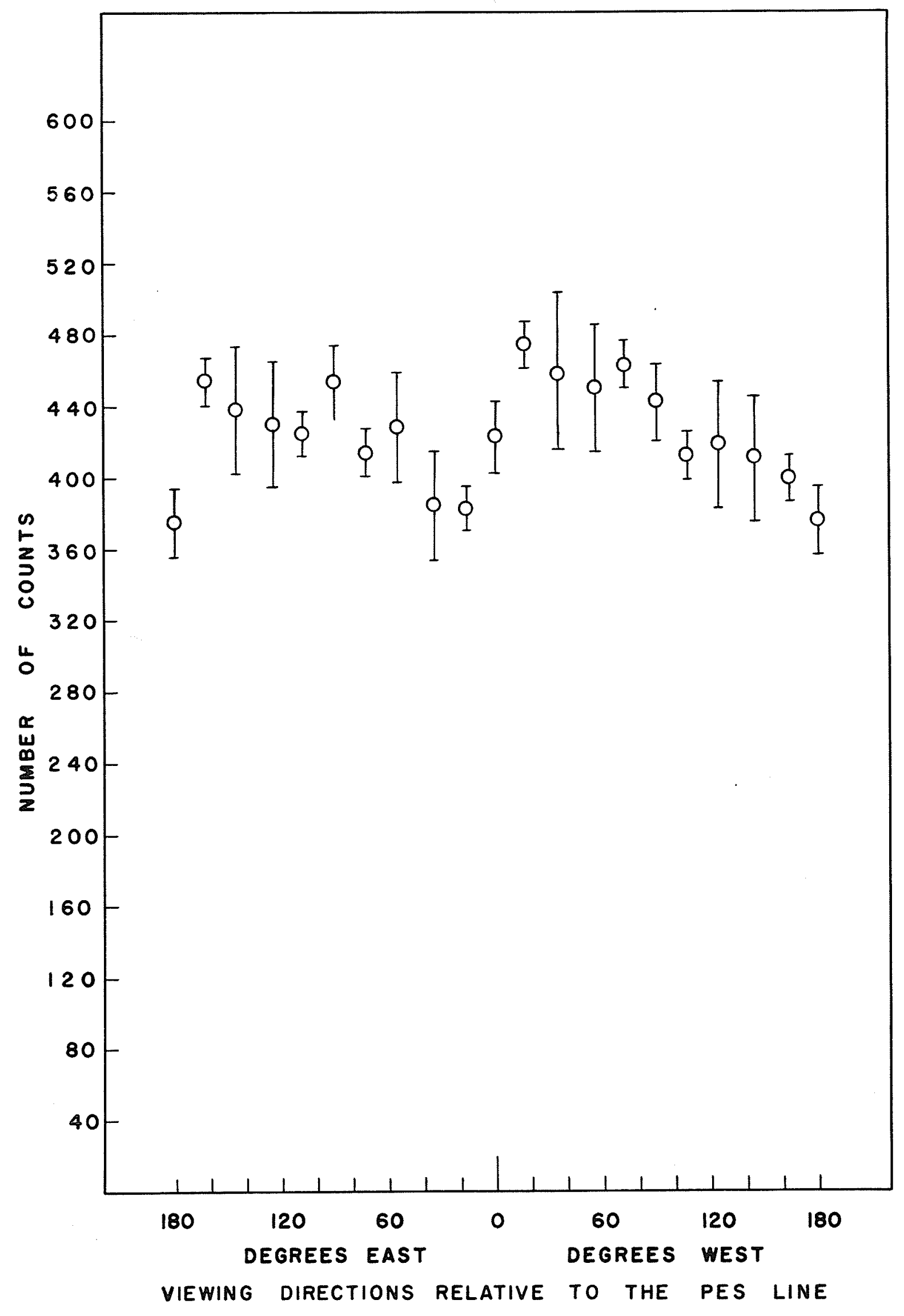


equator at various directions using the narrow angle telescopes. Due to the limited statistics of such a scan the counting time devoted to each particular direction was of necessity longer, the counting time for any direction being never less than six days. The observed counting rates (normalized to 6 days) as a function of PES direction are shown in figure 22 where an anisotropy of $(10 \div 3) \%$ is suggested. However, since only one scan was made not too much emphasis should be placed on these data, their presentation being mainly for completeness. However, during this interval the viewing direction $17.5^{\circ} \mathrm{E}$ continued to show a peak displacement to an earlier time.

The wide angle data collected during the short-term scans since February 29, however, do show a striking phenomenon. The portion of maximum intensity which was formerly evident at about $15^{\circ} \mathrm{W}$ has gradually wandered westward amongst the PES directions. Figures $23(a)$ and $23(b)$ show the nature of all the wide angle scans of the celestial equator. The data have been combined into sets of two consecutive scans (taking approximately 2 weeks to obtain) and presented for times between December 25, 1963 and July 23, 1964. Approximate curves have been drawn through the plotted points and the positions of maximum counting rate (with a generous error) have been estimated. The majority of these curves are characterized by two features. First, the maximum counting 
FIGURE 23(a) and (b)

Variation of Observed Peak Area (in a total of 4 days) With Viewing Direction During Consecutive Two Wide Angle Scans of the Celestial Bquator 


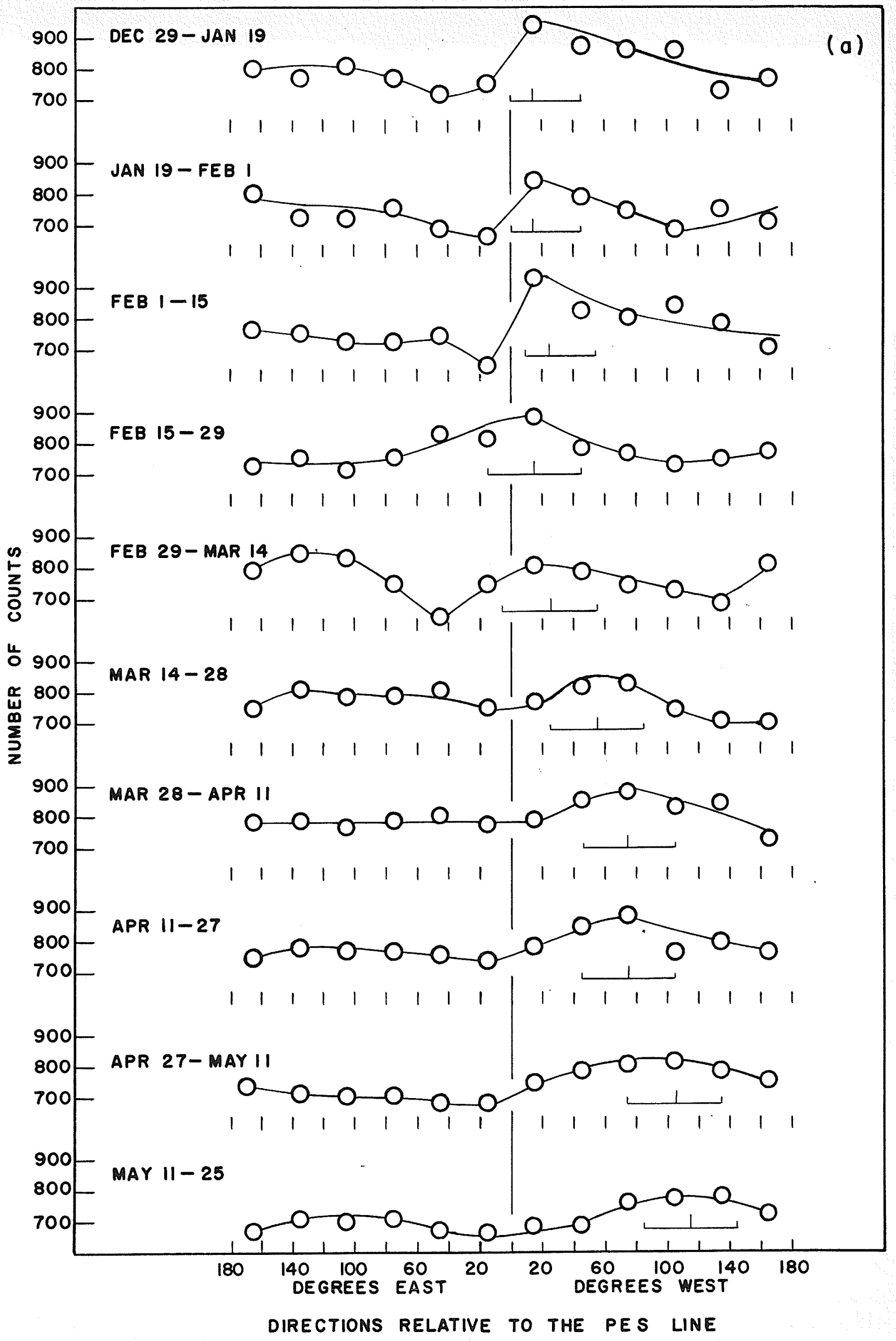


FIGURE 24

Variation of Observed Peak Area (in a total of 8 days) With Viewing Direction During Consecutive Four Wide Angle Scans of the Celestial Equator 


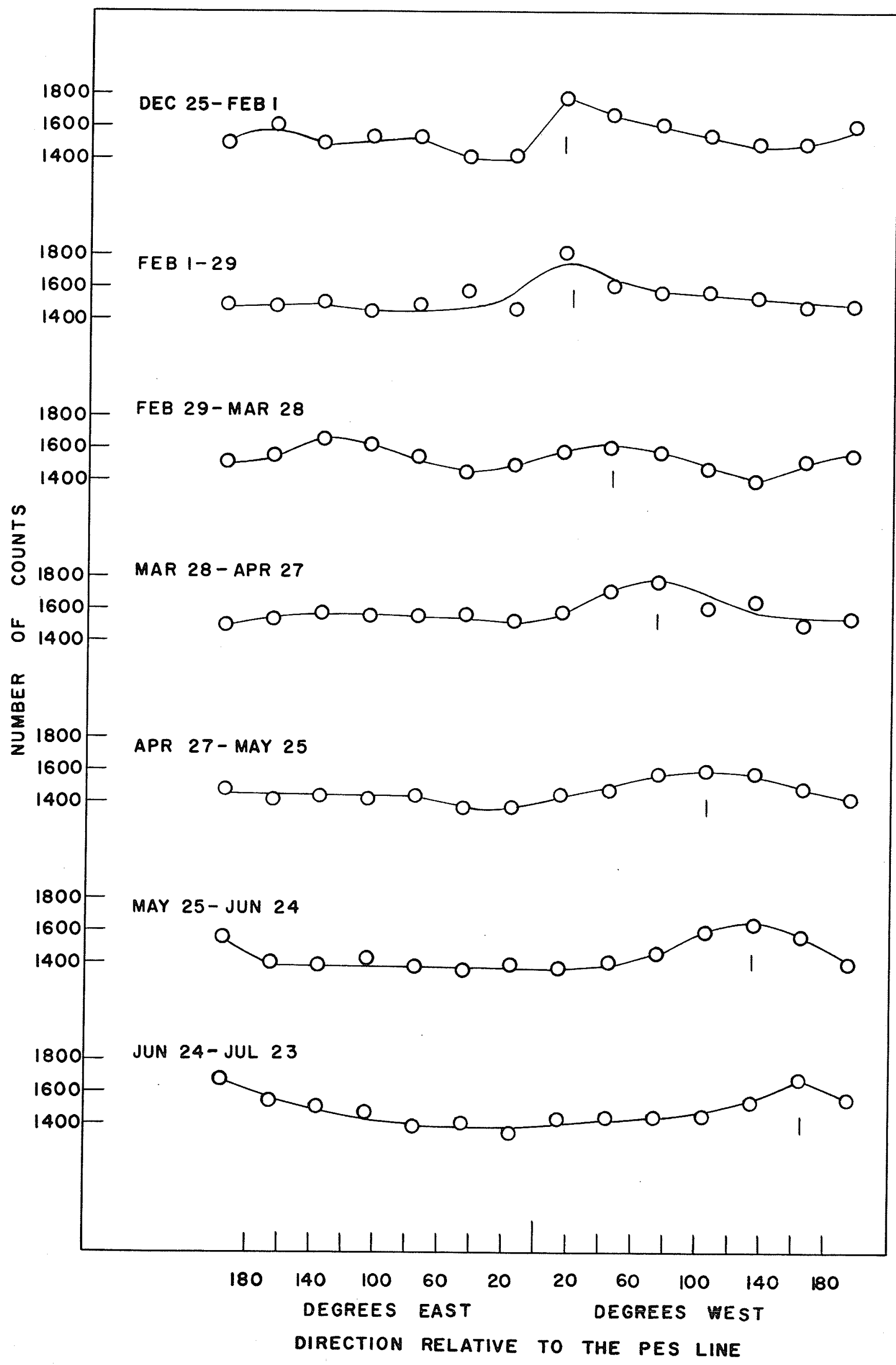


rate is seen to be slowly moving westward from $15^{\circ} \mathrm{W}$ in February to about $165^{\circ} \mathrm{W}$ in JuIy. Second, the viewing directions just east of the PES line are consistently suggestive of reduced counting rates in agreement with the results of the long-term run data from $17.5^{\circ} \mathrm{E}$. These same data have been smoothed out somewhat by similar plots of four scan totals and these curves are shown in figure 24. Again the westward trend of the position of maximum counting rate is evident. This westward movement is indicative of a beam of radiation approaching the earth from a direction in space fixed with respect to the plane of the celestial equator. As the earth rotates about the sun annually, the PES line rotates at a rate of $360^{\circ} / 12$ months or $30^{\circ}$ per month. Thus, if a beam of radiation were approaching the earth from a fixed galactic direction, its location with respect to the PIS line would move towards the west (as measured on earth) at the rate of $30^{\circ}$ per month. Figure 25 shows the position with respect to the PES line of maximum counting rate as a function of time. The open circles represent the estimated peak positions of the two-scan curves depicted in figure 23, while the full circles represent the estimated peak positions of the four-scan curves of figure 24. The points are plotted in the middle of the time interval during which the scans were made. The dashed line superimposed on the points is a straight line of $30^{\circ}$ per month slope passing as close as 
FIGURE 25

Position of Maximum Intensity as a Function of Time of Observation

Open Circles Represent Estimated Peak

Positions of Figure 23 
possible to the full circles. The agreement is very good. These data are then taken as evidence of the existence of a fairly intense beam of galactic cosmic radiation impinging upon the earth's atmosphere from a fixed direction within the galactic sphere. From the line of $30^{\circ}$ per month slope in figure 25, the peak position is taken to occur at $0^{\circ} \mathrm{W}$ on or about January 26. The anisotropy is then taken (from the SCl Chart) to be defined by an hour circle location of about $(20.5 \pm 1.0) \mathrm{h}$, a region roughly between the Constellations Aquarius and Aquila. This indication of a preferred arrival direction of cosmic radiation is in fair agreement with the results of the long-term narrow angle run which displayed enhanced counting rates in the region $21-23 \mathrm{~h}$.

During the first 8 scans (December 25 - February 29) the sun was roughly between the detector system and the galactic 'source'. This may account for the apparent constancy of the position of maximum intensity observed during that data collecting interval. (The maximum intensity remained around $15^{\mathrm{O} W}$ )

The counting rates observed during the 28 sweeps of the celestial equator are shown in Table 4.3. This table combines the collected data into quadrant totals, each quadrant containing three viewing directions. The quadrant $\left(180^{\circ}-90^{\circ}\right) \mathrm{E}$ includes the viewing directions $165^{\circ} \mathrm{E}, 135^{\circ} \mathrm{E}$ and $105^{\circ} \mathrm{E}$. The quadrant $\left(90^{\circ}-0^{\circ}\right) \mathrm{E}$ includes $75^{\circ} \mathrm{E}, 45^{\circ} \mathrm{E}$ and $15^{\circ} \mathrm{E}$. The 
$\left.10^{\circ}-90^{\circ}\right) \mathrm{W}$ quadrant includes $15^{\circ} \mathrm{W}, 45^{\circ} \mathrm{W}$ and $75^{\circ} \mathrm{W}$, while the quadrant $\left(90^{\circ}-180^{\circ}\right) \mathrm{W}$ includes $105^{\circ} \mathrm{W}, 135^{\circ} \mathrm{W}$ and $165^{\circ} \mathrm{W}$.

Evident from Table 4.3 is the consistency with which the $\left(90^{\circ}-0^{\circ}\right)$ E quadrant recorded counting rates lower than the other three.

TABLE 4.3

QUADRANT TOTALS FOR WIDE ANGLE SWEEPS OF THE CELESTIAL EQUATOR

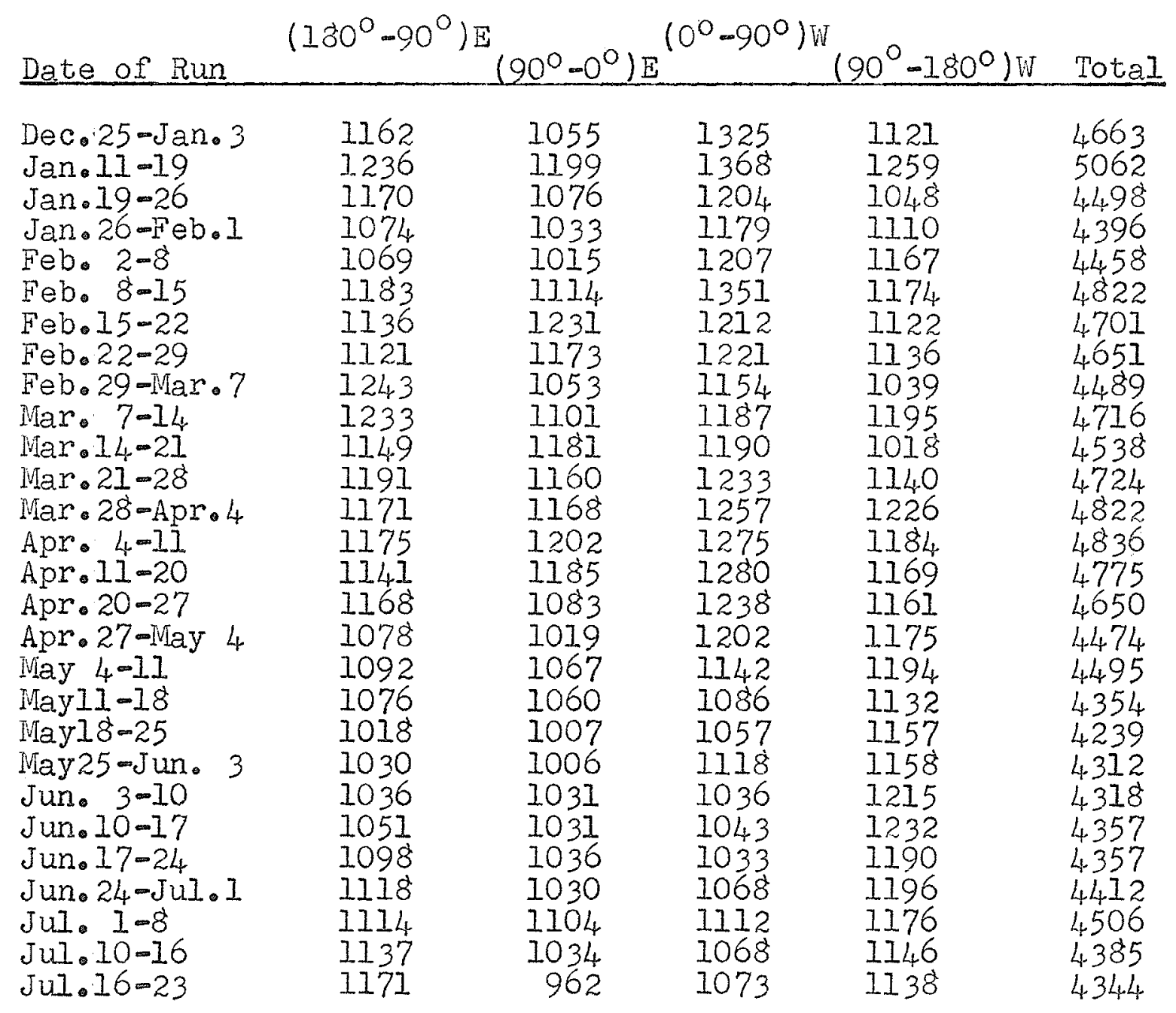


The data presented in this section are uncorrected for pressure changes because a consideration of the pressure effects did not result in a significant change in the nature of any of the data. As mentioned in Section 1.3, variations in the barometric pressure produce anticorrelated fluctuations in the ground level cosmic ray flux. This is seen from atmospheric absorption considerations. A falling barometer is indicative of a less dense absorbent layer for impinging cosmic radiation. Thus less radiation is absorbed and an increased ground level counting rate results. Similarly, a rising barometer indicates a denser absorbent atmosphere and a decreased counting rate results. The barometer coefficient $\beta$ is defined as the relative change in vertical cosmic ray intensity per unit change in barometric pressure. The coefficient for mesons approaching the detector from a zenith angle $\emptyset$ is increased by a factor sec $\emptyset$ due to the longer path length. The counting rate enhancement $\Delta C$ of the detector due to a pressure change is given by

$$
\Delta C=-\beta C \Delta P \sec \phi
$$

where $C$ is the observed counting rate at zenith angle $\phi, \Delta P$ is the difference between the pressure at which $C$ was observed and some reference pressure, and $\beta$ is the vertical barometer coefficient, generally expressed in units of percent per millibar.

Hourly reports on the barometric pressure were supplied 
to this laboratory daily by the Department of Transport, Winnipeg, and these were used to determine the extent of barometric influence on the counting rates obtained in this investigation. The analysis made was an hourly one. For the rotation of a telescope, an average hourly zenith angle $\phi$ was determined and an appropriate $\Delta C$ was calculated from the above formula using the corresponding values of $\mathrm{C}$ and $\Delta \mathrm{P}$ for that hour. A value for $\beta$ of $0.17 \%$ per mb reported by Wada (1960) was used. Arbitrarily $980 \mathrm{mb}$ (a value close to the average pressure at Winnipeg) was chosen as a reference pressure. Even during rapid pressure changes $\Delta P$ very rarely exceeded about $\pm 20 \mathrm{mb}$, the normal values being closer to between $\pm(1-10) \mathrm{mb}$. This hourly analysis was applied to the March and April data of the narrow angle run, the individual hourly corrections generally being of the order of \pm 0.02 counts. Very infrequently did the daily correction ever exceed $\pm(1-2)$ counts.

Similar calculations were made for the data collected during the wide angle runs, this time deliberately selecting those days during which $\Delta \mathrm{P}$ was the largest (i.e. days of rapid pressure changes). For example, collected data from the direction $15^{\circ} \mathrm{W}$ of the PES line was considered. During the months of December and January there were 8 days during which $\triangle \mathrm{P}$ was about $\pm(10-20) \mathrm{mb}$ (exceptionally large pressure increments). During this time about 1600 counts were compiled from $15^{\circ} \mathrm{W}$ while a pressure normalization to $980 \mathrm{mb}$ indicated that this number of counts should have been reduced by 8 
counts, representing a maximum correction of about $1 / 2 \%$. The normal day pressure effects are certainly considerably less. Thus pressure corrections alone would not be sufficient to explain observed anisotropies $\sim(4-19) \%$.

A possible explanation for the east-west anisotropy presented in the published results (Standil and Bukata, 1964c) was offered by Dr. M. Wada (1964) who suggested the observations might be caused by the positive temperature effect. Briefly, temperature effects are as follows: The negative temperature effect, as mentioned earlier, is explained on the basis of the instability of the $\mu$-meson. A positive temperature effect also exists, but this effect concerns the $\pi$-meson rather than the $\mu-m e s o n$. The $\pi$-meson formed in the upper reaches of the atmosphere is confronted with two competing processes, viz, atmospheric absorption and decay into $\mu$-mesons. With an increase in temperature, atmospheric density decreases, the probability of absorption drops, and the probability of decay is increased. This results in the creation of more muons and therefore a possible increased counting rate for muon detectors.

However, there are strong arguments against a temperature explanation. First, the existence of such a temperature effect would imply a strong dependence of counting rate on local time. The increased counting rates would therefore be not a function of viewing direction, but rather a function of local solar time. 
The counting rates, independent of associated viewing directions would be highest during the afternoon hours when the atmosphere is warmest and lowest during the night when the atmosphere is coolest. This possibility was considered in the following manner: A 'total' histogram for the 12 viewing directions depicted in figure 19 was first obtained by simply superimposing the twelve histograms and adding them point by point. This total histogram was then normalized to the appropriate number of days (24 days for directions $45^{\circ} \mathrm{W}$ and $135^{\circ} \mathrm{E}, 25$ days for directions $45^{\circ} \mathrm{E}$ and $135^{\circ} \mathrm{W}$ and 16 days for the other eight directions) and the resulting 'effective average' histogram was compared to the actual histogram obtained for each direction. The deviations of each individual histogram from the appropriate 'average' histogram were determined on an hourly analysis. These deviations were then converted into percentage deviations from the average histogram and plotted as a function of local time. A deviation curve was plotted for each viewing direction spanning the interval of local time during which a telescope sampled radiation from the particular direction considered. These curves are shown for easterly and westerly directions in figures $26(a)$ and 26(b) respectively. From the nature of these curves it is apparent that there is no strong time dependence of enhanced counting rate. Thus an explanation for the observed anisotropy in terms of temperature effects is unlikely. 
Deviation From Average Histogram as a Function of IST

(a) Easterly Directions During First Eight Scans

(b) Westerly Directions During First Eight Scans

(c) Sample Directions During an Intermediate Eight Scans 


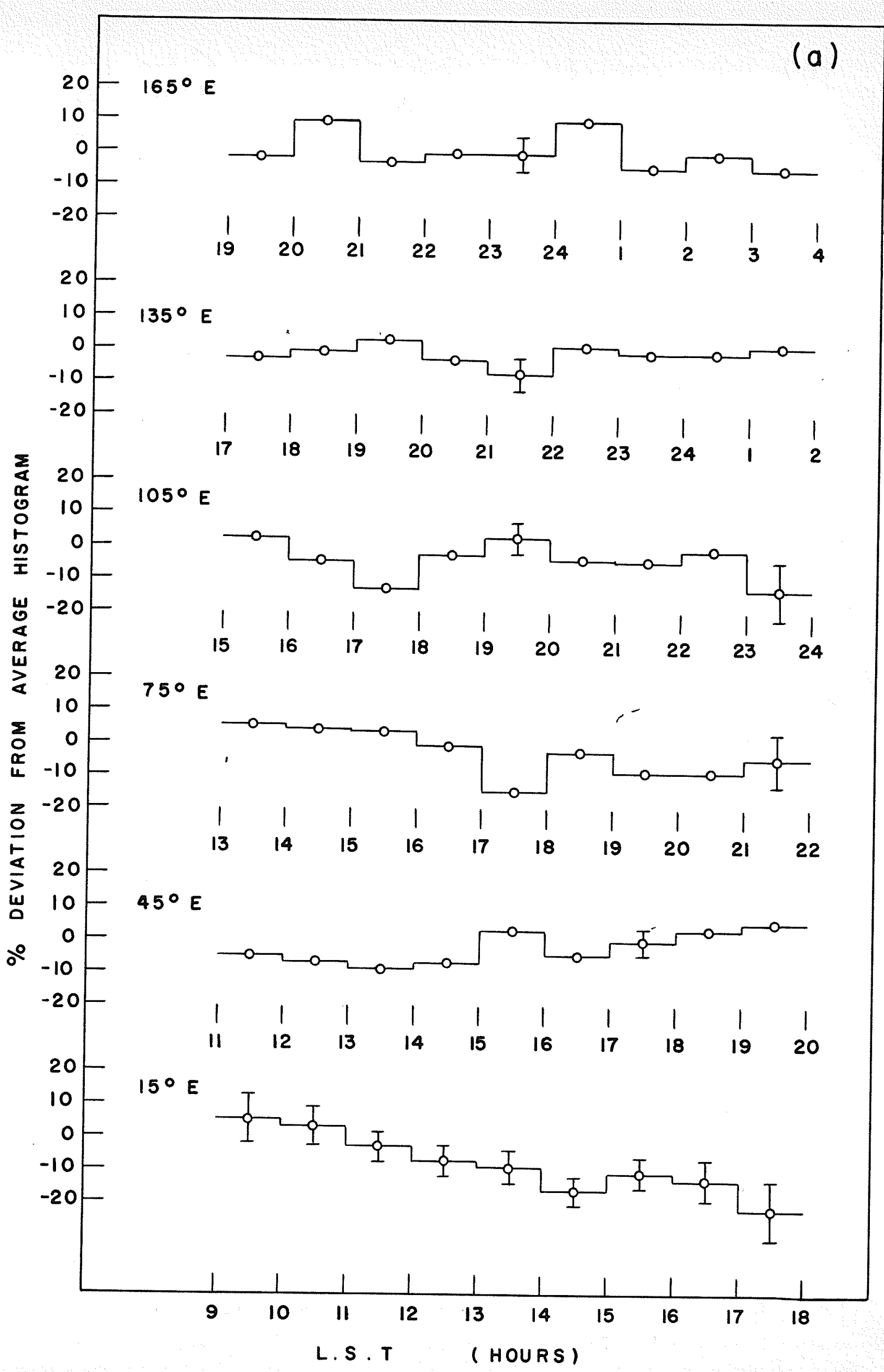




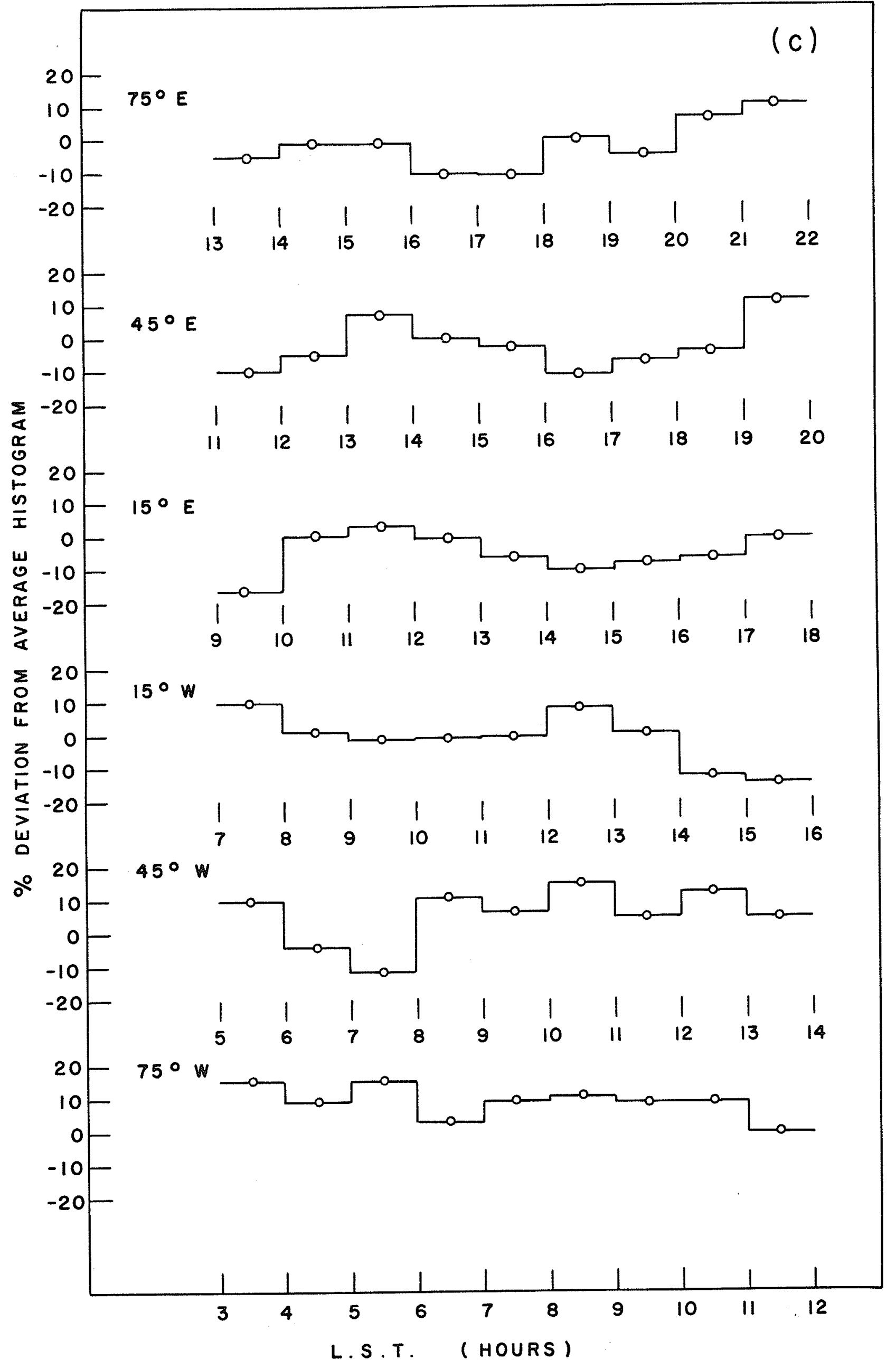


Further, since the directions $15^{\circ} \mathrm{E}$ and $15^{\circ} \mathrm{W}$ are centred on $13: 30$ and $11: 30 \mathrm{LST}$ respectively, this would mean the two hours between 11:30 and 13:30 must be consistently the daily times of maximum atmospheric temperature, with 12:30 the hottest portion of the day. In reality the average temperature maximum occurs later in the afternoon, closer to 15:00 LST. Also, since rapid cooling occurs during the night, it might be expected, on the same reasoning, that the directions $135^{\circ} \mathrm{E}$ to $135^{\circ} \mathrm{W}$ would show definite intensity decreases of about the same order of magnitude as the midday increases. No such consistent intensity decreases were detected.

The strongest argument against the temperature explanation is the westward movement of the direction of maximum intensity with time shown in figures 23 to 25. The intensity maximum shifted very nearly $30^{\circ}$ per solar month, corresponding to a daily intensity maximum shift towards later solar hours. For example, a maximum counting rate at, say, $135^{\circ} \mathrm{W}$ corresponds to a histogram centred at 03:30 LST, certainly not an expected hour of maximum atmospheric temperature. In figures $26(a)$ and $26(b)$ the behaviour of the deviation curves for $15^{\circ} \mathrm{E}, 15^{\circ} \mathrm{W}$, and $45^{\circ} \mathrm{W}$ is noted. These curves span roughly comparable local time intervals between 0500 and 1800 LST, each curve including local noon. However, with increasing local time, the curve for $15^{\circ} \mathrm{E}$ displays a negative slope, going from positive to negative deviations. The curve for 
$45^{\circ} \mathrm{W}$ displays a positive slope, rising from negative deviations to positive deviations. The curve for $15^{\circ} \mathrm{W}$ is consistently very high, independent of the local time. These curves represent the solar modulation of the galactic cosmic radiation approaching the earth from the vicinity of the sun as discussed earlier. The negative slope for $15^{\circ} \mathrm{E}$ is indicative of a histogram peak shift to earlier hours (i.e. eastward from the viewing direction). This peak shift coupled with the reduction in integrated area is consistent with a solar mechanism which removes particles from directions east of $15^{\circ} \mathrm{E}$ of the PES line and/or increases the number of particles from the west. Similarly, the positive slope for $45^{\circ} \mathrm{W}$ is indicative of a peak shift to later hours (i.e. westward from the viewing direction). This is suggestive of increased intensity from a direction east of $45^{\circ} \mathrm{W}$. These two results suggest an anisotropy in the cosmic radiation with a maximum intensity between $15^{\circ} \mathrm{E}$ and $45^{\circ} \mathrm{W}$ of the PES line. The viewing direction $15^{\circ} \mathrm{W}$ is midway between the se two directions and it is significant that the deviation curve for $15^{\circ} \mathrm{W}$ shows no sign of a peak shift either eastward or westward, and that the histogram is characterized by consistent increased counting rates throughout. During these 8 scans (December 25 - February 29) the region of the celestial sphere near $20.5 \mathrm{~h}$ (the location of the galactic 'source' observed during the short-term runs) in relation to the PES line moved from about $25^{\circ} \mathrm{E}$ to about 
$30^{\circ} \mathrm{W}$. It is possible that the sun, due to its orientation between the detector and the galactic 'source' caused the observed decrease in easterly intensity and increase in westerly intensity.

Similar analyses (deviations from an 'average' histogram) were carried out on the 8 scan interval between March 23 and May 18 when the position of maximum intensity had moved out to $\propto 75^{\circ} \mathrm{W}$. The deviation curves (some of which are shown in figure $26(\mathrm{c}))$ obtained for this interval are also not suggestive of a dependence of enhanced counting rate on local time. The curves for $45^{\circ} \mathrm{W}$ and $75^{\circ} \mathrm{W}$ are both consistently high due to the increased counting rates observed from these viewing directions as the position of maximum intensity moved westward. The curves for $15^{\circ} \mathrm{E}$ and $45^{\circ} \mathrm{W}$, however, do not have the definite negative and positive slopes of figures $26(a)$ and 26(b). During these \& scans the earth had moved such that the sun no longer occupied a position between the detectors and the galactic 'source'. With this change of position, the solar modulation of the radiation from the galactic 'source' became less prominent. This is seen from Table 4.4 in which are tabulated the values of the anisotropy

$$
\delta=\frac{\operatorname{Imax}-\operatorname{Imin}}{\operatorname{Imax}+\operatorname{Imin}} \times 100 \%
$$

calculated from each of the fourteen curves of figures $23(a)$ 
and $23(b)$. Table 404 shows the values of $\delta$ along with the time intervals they were observed.

\section{TABLE 4.4}

OBSERVED ANISOTROPY \& CALCULATED FROM EACH OF THE CURVES OF FIGURES 23(a) AND 23(b)

\section{Date of Observation}

Dec.25-Jan. 19

Jan. $19-F$ eb. 1

Feb. 2-15

Feb.15-29

Feb.29-Mar.14

Mar. $14-28$

Mar.28-Apr.11

Apr. II $=27$

Apr.27-May 11

May $11-25$

May 25-Jun. 10

Jun. $10-24$

Jun. 24-Jul. 8

Jul. $8-23$
Anisotropy \& $(\%)$

$13.3 \pm 3.5$
$11.9 \pm 3.7$
$13.9 \pm 3.5$
$10.4 \pm 3.6$
$10.8 \pm 3.7$
$4.7 \pm 3.6$
$4.6 \pm 3.4$
$8.8 \pm 3.5$
$8.6 \pm 3.6$
$7.9 \pm 4.0$
$8.5 \pm 3.6$
$9.4 \pm 3.6$
$8.0 \pm 3.6$
$9.7 \pm 3.6$

Despite the large uncertainty associated with each $\delta$, it is seen from Table 4.4 that anisotropies are smaller after March 14. The largest values of $\delta$ occurred during the time that the sun was between the galactic 'source' and the detectors.

Assuming an intensity-zenith angle variation law $j(\phi)=j(0) \cos ^{n} \phi$, an attempt was made to estimate the exponent n. All the data collected during both the narrow angle and wide angle runs were utilized.

Taking the logarithm of both sides of the zenith variation law results in the equation 


$$
\log j(\phi)=\log j(0)+n \log \operatorname{Cos} \phi
$$

This equation represents a straight line plot of log $j(\emptyset)$ values against corresponding $\log \cos \phi$ values. The slope of this straight line is $n$ and $\log j(0)$ is the intercept at $\phi=0^{\circ}$. All histograms were analyzed in 20 -minute intervals over an $82 / 3$ hour period centred on the detector orientation of maximum counting rate. Since $\phi$ decreases monotonically from $74.2^{\circ}$ to $49.9^{\circ}$ and then increases through an identically reflected sequence back to $74.2^{\circ}$ for the histogram portion considered, the above equation is really the equation of a pair of straight Iines, one for the rising portion of the histogram and one for the falling. In the 20 -ninute analysis the number of counts in a 20-minute interval was determined, and this number recorded along with the average zenith angle orientation of the detector during that time interval. Both the falling and rising segments of the histogram were thus defined by fourteen such $(\phi, j(\phi))$ pairs. A least squares fit was then applied to both the rising and falling segments of each histogram in question to determine the slope and intercept. This was accomplished by setting up a program for the IBM 1620 computer to analyze the collected data. 
Table 4.5 shows the values of $j(0)$ and $n$ obtained from the data of the narrow angle runs (March I, 1963 - November I, 1963) for both the rising and falling portions of monthly histograms obtained at $107.5^{\circ} \mathrm{E}, 162.5^{\circ} \mathrm{W}$ and $72.5^{\circ} \mathrm{W}$. Because of the obvious peak shift for the viewing direction $17.5^{\circ} \mathrm{B}$, this direction was not considered in the least squares analysis. 


\section{TABLE 4.5}

\section{LEAST SQUARES ANALYSIS FOR $n$ AND $j(0)$} (NARROW ANGLE RUNS)

(a) $107.5^{\circ} \mathrm{E}$

$\begin{array}{lccll}\text { Date } & \underline{n \text { (Rise) }} & \underline{n \text { (Fall) }} & \underline{j(0) \text { (Rise) }} & \underline{j(0) \text { (Fall) }} \\ \text { March } & 0.63 \pm .14 & 1.67 \pm .12 & 4.2 \pm 0.5 & 6.0 \pm 0.6 \\ \text { April } & 1.07 \pm .14 & 1.85 \pm .10 & 5.2 \pm 0.6 & 7.0 \pm 0.6 \\ \text { May } & 0.87 \pm .15 & 1.30 \pm .15 & 4.6 \pm 0.6 & 5.3 \pm 0.6 \\ \text { June } & 1.28 \pm .14 & 1.56 \pm .12 & 5.5 \pm 0.7 & 5.6 \pm 0.6 \\ \text { July } & 1.05 \pm .11 & 1.36 \pm .15 & 5.2 \pm 0.5 & 5.5 \pm 0.8 \\ \text { August } & 1.51 \pm .08 & 1.50 \pm .09 & 6.6 \pm 0.5 & 6.1 \pm 0.4 \\ \text { Sept. } & 1.14 \pm .19 & 1.52 \pm .10 & 4.8 \pm 0.8 & 5.3 \pm 0.4 \\ \text { Oct. } & 1.05 \pm .13 & 1.37 \pm .17 & 4.7 \pm 0.5 & 4.5 \pm 0.7\end{array}$

(b) $162.5^{\circ} \mathrm{W}$

$\begin{array}{llllll}\text { March } & 0.81 \pm .10 & 1.54 \pm .17 & 4.4 \pm 0.4 & 5.8 \pm 0.9 \\ \text { April } & 1.35 \pm .12 & 1.38 \pm .13 & 6.0 \pm 0.6 & 5.9 \pm 0.7 \\ \text { May } & 0.94 \pm .11 & 1.05 \pm .15 & 4.6 \pm 0.4 & 4.8 \pm \pm 0.6 \\ \text { June } & 1.17 \pm .13 & 1.22 \pm .16 & 4.9 \pm 0.5 & 4.5 \pm 0.7 \\ \text { JuIy } & 1.51 \pm .13 & 0.88 \pm .11 & 6.1 \pm 0.7 & 4.1 \pm \pm 0.4 \\ \text { August } & 1.38 \pm .10 & 1.08 \pm .14 & 5.4 \pm 0.5 & 4.5 \pm 0.5 \\ \text { Sept. } & 1.39 \pm .13 & 1.17 \pm .12 & 6.0 \pm 0.6 & 4.9 \pm \pm 0.5 \\ \text { Oct. } & 1.20 \pm .10 & 1.40 \pm .20 & 5.0 \pm 0.4 & 5.4 \pm 0.6\end{array}$

(c) $72.5^{\circ} \mathrm{W}$

$\begin{array}{llllll}\text { March } & 1.08 \pm .13 & 1.44 \pm .19 & 5.0 \pm 0.6 & 5.3 \pm 0.9 \\ \text { April } & 1.23 \pm .13 & 1.41 \pm .12 & 5.7 \pm 0.6 & 5.3 \pm 0.5 \\ \text { May } & 1.31 \pm .12 & 1.19 \pm .16 & 5.9 \pm 0.5 & 4.9 \pm 0.7 \\ \text { June } & 1.38 \pm .13 & 1.50 \pm .15 & 5.6 \pm 0.6 & 5.7 \pm 0.8 \\ \text { JuIy } & 1.14 \pm .10 & 1.07 \pm .14 & 4.8 \pm 0.4 & 4.5 \pm 0.5 \\ \text { August } & 1.40 \pm .12 & 1.42 \pm .15 & 5.8 \pm 0.6 & 5.5 \pm 0.7 \\ \text { Sept. } & 1.51 \pm .13 & 1.51 \pm .10 & 5.9 \pm 0.6 & 6.0 \pm 0.5 \\ \text { Oct. } & 1.18 \pm .13 & 1.02 \pm .15 & 4.7 \pm 0.5 & 4.2 \pm 0.5\end{array}$

Table 4.6 shows the results of similar analysis performed on the total data collected from these same three directions. 


\section{TABLE 4.6}

\section{LEAST SQUARES ANALYSIS FOR $n$ AND $j(0)$} (NARROW ANGLE RUNS)

Viewing

\begin{tabular}{|c|c|c|c|c|c|c|}
\hline Direction & $n$ (Rise) & & $n(\mathrm{Fall})$ & & $j(0)$ (Rise) & $j(0)(\mathrm{Fall})$ \\
\hline $\begin{array}{l}162.5 \mathrm{OW} \\
72.5 \mathrm{~W}^{2}\end{array}$ & $\begin{array}{l}1.04 \pm \\
1.20 \pm \\
1.27 \pm\end{array}$ & $\begin{array}{l}.05 \\
.04 \\
.04\end{array}$ & $\begin{array}{l}1.51 \pm \\
1.20 \pm \\
1.31 \pm\end{array}$ & $\begin{array}{l}.06 \\
.07 \\
.08\end{array}$ & $\begin{array}{l}5.0 \pm 0.2 \\
5.2 \pm 0.2 \\
5.4 \pm 0.2\end{array}$ & $\begin{array}{l}5.7 \pm 0.3 \\
5.0 \pm 0.3 \\
5.2 \pm 0.4\end{array}$ \\
\hline
\end{tabular}

Table 4.7 shows the values of $n$ and $j(0)$ obtained when all the data collected during the long-term run was combined. An effective 'average' histogram was considered by superimposing the histograms for $107.5^{\circ} \mathrm{E}, 162.5^{\circ} \mathrm{W}$ and $72.5^{\circ} \mathrm{W}$ and adding them point by point. A least squares analysis was then performed on such an average histogram and the monthly results are recorded in the table. The final entry represents the combined effect of all three directions taken over the entire 8 month interval.

\section{TABIE 4.7}

LEAST SQUARES ANALYSIS FOR $n$ AND $j(0)$ (COMBINED DATA FROM 107.5

\begin{tabular}{|c|c|c|c|c|}
\hline te & $n$ (Rise) & $\mathrm{n}(\mathrm{FaII})$ & (0)(Rise) & $\mathrm{iO})(\mathrm{Fall}$ \\
\hline $\begin{array}{l}\text { reh } \\
\text { ril } \\
\text { ane } \\
\text { aly } \\
\text { agust } \\
\text { pt. }\end{array}$ & $\begin{array}{l}0.81 \pm .06 \\
1.19 \pm .06 \\
1.02 \pm .08 \\
1.27 \pm .09 \\
1.21 \pm .07 \\
1.43 \pm .07 \\
1.34 \pm .08 \\
1.14 \pm .08\end{array}$ & $\begin{array}{l}1.53 \pm .11 \\
1.52 \pm .08 \\
1.17 \pm .10 \\
1.41 \pm .10 \\
1.09 \pm .10 \\
1.32 \pm .09 \\
1.39 \pm .08 \\
1.24 \pm .14\end{array}$ & $\begin{array}{l}4.5 \pm 0.2 \\
5.6 \pm 0.3 \\
4.9 \pm 0.3 \\
5.3 \pm 0.4 \\
5.3 \pm 0.3 \\
6.0 \pm 0.3 \\
5.5 \pm 0.3 \\
4.8 \pm 0.3\end{array}$ & $\begin{array}{l}5.7 \pm 0.5 \\
6.0 \pm 0.5 \\
5.0 \pm 0.5 \\
5.2 \pm 0.4 \\
4.7 \pm 0.4 \\
5.3 \pm 0.4 \\
5.4 \pm 0.4 \\
4.6 \pm 0.5\end{array}$ \\
\hline 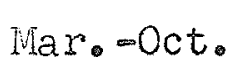 & $17 \pm$ & $.33 \pm .06$ & $.2 \pm 0.1$ & $.2 \pm 0.3$ \\
\hline
\end{tabular}


The nature of the final entry in Table 4.7 prompted the use of the function $j(D)=5.2 \cos ^{1.25} \phi$ as the comparison curve superimposed on the histograms in figure 14. This value of $\mathrm{n}$ (1.25) was taken as the average of the $\mathrm{n}$ (Rise) and $\mathrm{n}(\mathrm{FaII})$ values which appear to be statistically different. It is noted that for the months of March and May the $n$ (Rise) values were considerably lower than those for the other six months. The significance of this is not clearly understood.

The wide angle data collected during the short term runs which began December 25, 1963 were treated in a manner similar to the treatment used in obtaining Table 4.7. In this case analysis was made on each complete scan of the celestial equator. The 'average' histogram obtained during that scan from all 12 viewing directions was considered. Since any peak shifts that might exist have been considerably reduced by the wider angle, no viewing directions have been removed from the analysis, all twelve directions receiving equal weight. Table 4.8 illustrates the appropriate rising and falling $n$ and $j(0)$ values obtained for each individual scan of the celestial equator. 


\section{TABLE 4.8}

LEAST SQUARES ANALYSIS FOR $n$ AND $j(0)$

(INDEPENDENT WIDE ANGLE SCANS OF THE CELESTIAL EQUATOR)

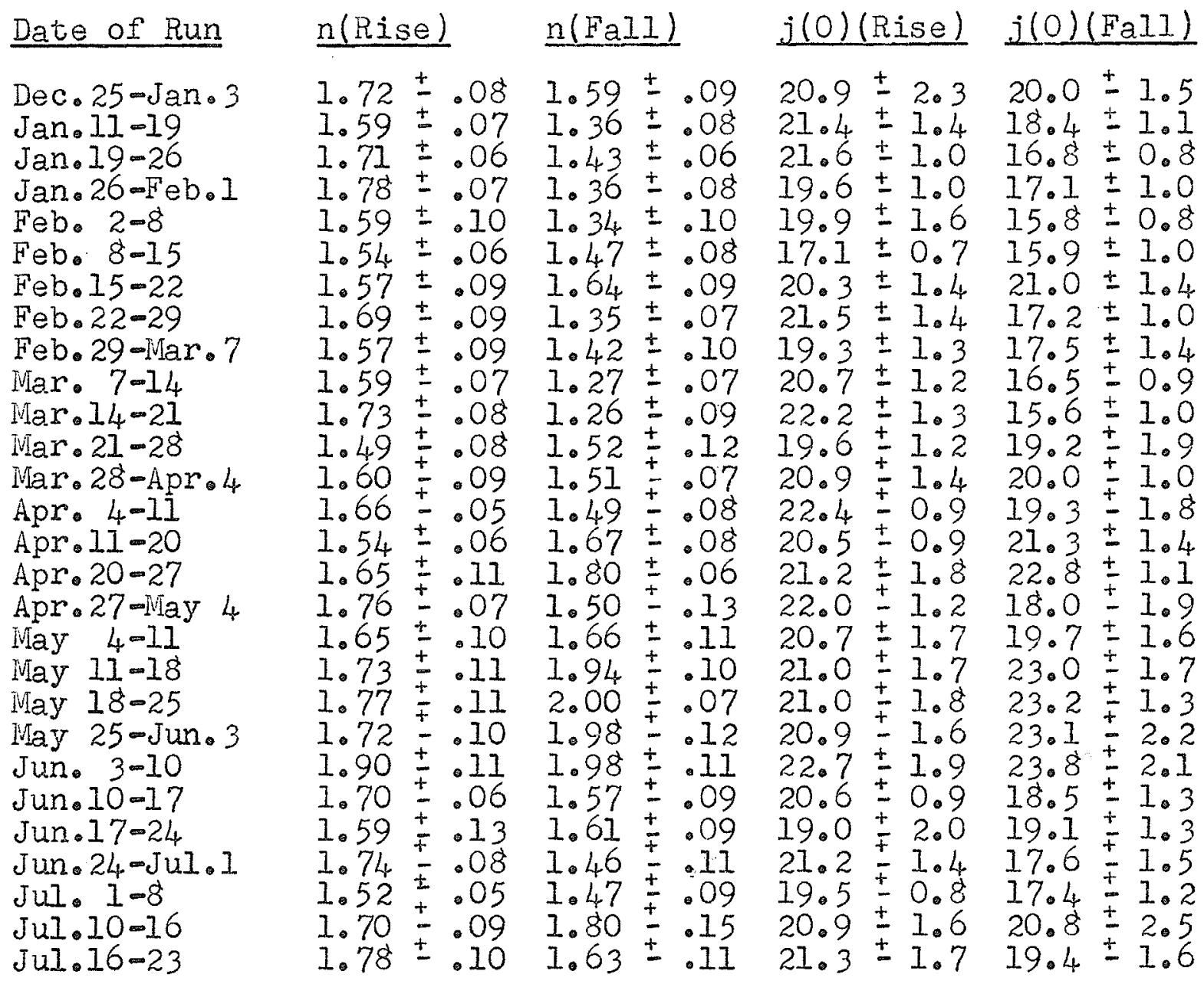

Combining several wide angle scans to improve statistics resulted in the values quoted in Table 4.9.

\section{TABIE 4.9}

LEAST SQUARES ANALYSIS FOR $n$ AND $j(0)$ (WIDE ANGLE SCANS)

$\begin{array}{lllll}\text { Date of Run } & \text { n(Rise) } & \frac{n(F a l l)}{j(0)(R i s e)} & j(0)(F a I l) \\ \text { Dec.25-Feb.29 } & 1.63 \pm .03 & 1.45 \pm .06 & 20.8 \pm 0.6 & 18.4 \pm 0.8 \\ \text { Mar.2l-May I8 } & 1.63 \pm .04 & 1.61 \pm .06 & 21.0 \pm 0.5 & 20.1 \pm 0.9\end{array}$


TABLE 4.9 (Cont.)

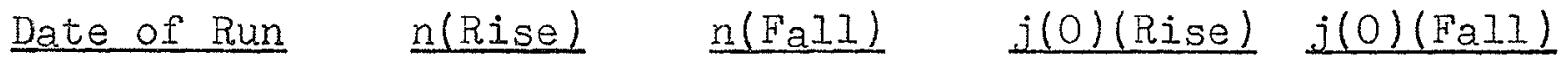

Dec.25-Jun. $3 \quad 1.64 \pm .03 \quad 1.54 \pm .05 \quad 19.6 \pm 0.4 \quad 18.0 \pm 0.6$ Jun. $3-J_{u l} 16 \quad 1.68 \pm .05 \quad 1.63 \pm .07 \quad 20.6 \pm 0.8 \quad 19.3 \pm 1.1$ Dec.25-Ju1.16 $1.65 \pm .03 \quad 1.56 \pm .05 \quad 20.8 \pm 0.5 \quad 19.2 \pm 0.8$

From the values in Table 4.9 a comparison function $j(\phi)=20.0 \cos ^{1.60} \phi$ was chosen as the equation of best fit to the data collected with the wide angle telescopes and this function appears superimposed on the histograms of figure 19.

It is noted that the values of $\mathrm{n}$ for the narrow angle runs were close to about 1.25 while the wide angle runs gave a value of $\mathrm{n}$ about 1.60. Although the reasons for this difference in $n$ values are not clearly understood, the following factors could have contributed to producing an exponent $\mathrm{n}$ for the narrow angle run which was smaller than the corresponding n for the wide angle run.

The random shower coincidences have only been partially eliminated. Unwanted coincidences contributed to the counting rates of the telescopes, the relative contribution being greater at higher zenith angles. Since the counting rates of the narrow angle telescopes were considerably lower than the counting rates of the wide angle telescopes, unwanted coincidences would affect the narrow angle telescopes to a greater extent than the wide angle telescopes. This would result in broader (lower $n$ ) experimentally determined histograms for the 
narrow angle telescopes. Also, the removal of $\mathrm{Pb}$ absorber from a telescope lowers the minimum energy of the particles detected by that telescope. For the narrow angle run a $\mu$-meson required $\sim 0.8 \mathrm{Bev}$ of energy to penetrate the $\mathrm{Pb}$ absorber. For the wide angle runs this muon energy was reduced to $\sim 0.4 \mathrm{Bev}$. The exponent $\mathrm{n}$ is a function of muon energy (as mentioned in Section 1.2), decreasing with increasing energy. Thus the smaller $n$ value for the narrow angle run is consistent with the higher energy muons detected during that run. This difference in energies of detected muons also explains the different $j(0)$ values for the narrow and wide angle runs. Although the coincidence solid angle for the wide angle telescope was about 3 times that of the narrow angle telescope, the value of $j(0)$ increased by a factor of about 3.8 from about 5.2 to about 20.0 .

4.2 CONCLUSIONS

The conclusions based upon the experimental data presented in the preceding section may be summarized as follows:

The steady stream of galactic radiation impinging upon the earth's surface is not isotropic. With the aid of narrow angle, directionally selective, rotating telescopes, large anisotropies of the primary radiation arriving in or near the equatorial plane of the celestial sphere have been observed. Preferred arrival directions appear to exist, the most prominent of which is defined by a RA of about $(20.5 \pm 1.0)$ h, a direction which is close to the heart of the galaxy (the Sagittarius arm)。 
Other possible preferred directions are defined by $\mathrm{RA} \sim 10.0 \mathrm{~h}, 16.0 \mathrm{~h}$, and $4.0-6.0 \mathrm{~h}$.

Evidence exists which suggests a modulation of the primary cosmic radiation as a result of its interaction with the inner solar system. This shows up in comparing observations made from directions just east and just west of the PES line. In addition to reduced intensities from directions just east of the PES line, peak shifts are observed on the time-of-arrival histograms, thereby indicating departures from a $\cos ^{n} \phi$ zenith variation law. It is suggested that the sun's associated 'garden hose' magnetic field may be largely responsible. 
$\underline{P A R T \quad F I V E}$ APPENDICES 
APPENDIX I - ESTIMATE OF THE MINIMUN PROTON ENERGY

The problem of estimating the minimum energy proton detected by the telescopes was considered in three stages. First the minimum $\mu$-meson energy detected by the telescopes was estimated, then this value was used to estimate the energy of a pion required to produce such a muon, and finally the pion energy was used to evaluate the minimum proton energy required to produce this pion.

From range-energy curves (Leighton, 1959), a $\mu$-meson required about $0.8 \mathrm{Bev}$ of energy to penetrate $22^{\prime \prime}$ of $\mathrm{Pb}$ absorber. If it is assumed that most of the muons were produced at the $100 \mathrm{mb}$ level (i.e. after traversing 10\% of the atmosphere), then the mesons approaching the earth from the vertical direction travelled through $90 \%$ of the atmosphere (i.e. $900 \mathrm{~g} \mathrm{~cm}^{-2}$ of atmospheric absorber) before reaching the $\mathrm{Pb}$. However, a meson telescope was never directly vertical, its most vertical orientation being at a zenith angle of $49.9^{\circ}$. Therefore, the minimum effective atmospheric length encountered by a muon was $900 \mathrm{sec} 49.9^{\circ} \approx 1300 \mathrm{~g} \mathrm{~cm}^{-2}$. The energy loss by collisions of muons in an air absorber was about $2 \mathrm{Mev}$ per $\mathrm{g} \mathrm{cm}^{-2}$ (Thorndike, 1952). Hence, to penetrate the atmosphere the meson possessed an energy of at least $2600 \mathrm{Mev}$. Then, allowing for muon energy loss in the detectors, a conservative estimate of muon energy was about $3.5 \mathrm{Bev}$. (That is, a meson telescope detected muons whose energy at production was at least $3.5 \mathrm{Bev}$. ) 
These $\mu$-mesons were predominantly produced by the decay in flight of $\pi$-mesons.

$$
\pi \longrightarrow \mu+\gamma
$$

If the decay is considered from the rest frame of the $\pi-$ meson, the $\mu$-meson and the neutrino must move in opposite directions after the decay occurs. In the laboratory frame of reference, however, the $\mu$ and $y$ may move in the same direction, opposite directions, or at some angle to one another. So as to be observed by the detectors, the $\mu$-meson was assumed to be emitted in the forward direction in the laboratory system. Two extreme cases were considered .

Case 1): The meson and the neutrino move oppositely to one another in the laboratory frame of reference.

Application of the conservation principles of relativistic energy and relativistic momentum showed that the energy $E_{\mu}$ of the emitted $\mu-m e s o n$ was equal to the energy $E_{\pi}$ of the original $\pi$-meson. The neutrino was created at rest. Hence $E_{\pi}=E_{\mu}=3.5 \mathrm{Bev}$ in the laboratory system for this case.

Case 2): The meson and the neutrino move in the same direction in the laboratory frame of reference.

In this case it was found that the neutrino carried away nearly $44 \%$ of the initial T-meson energy and $E_{\pi}=6.2$ Bev as measured in the laboratory。

The relativistic production of $\pi$-mesons was next considered 
in relation to the primary proton energy. The reaction was essentially a nucleon-nucleon collision (proton with an atmospheric nucleon). The problem was further complicated by the multiplicity of pion production at high proton energies. Lorentz transformations were used to relate the collision conditions as observed from the centre of mass reference frame of the two nucleons and the laboratory reference frame. A probable pion energy (centre of mass reference frame) deter mined from a curve presented by Perkins (1961) was used in the calculations. The calculations showed that to produce a pion of laboratory energy 3.5 Bev (Case I) a primary proton of laboratory energy 36.8 Bev was required, while to produce a pion of laboratory energy 6.2 Bev (Case 2) a primary proton of. laboratory energy $106 \mathrm{Bev}$ was required. These calculations assumed that the pion was emitted in the forward direction in the centre of mass system. Similar computations were carried out for the case of the pion emitted backwards in the centre of mass system. The required laboratory proton energies for this case were much higher than those for the case of forward pion emission, being approximately $6.53 \times 10^{3}$ Bev for Case 1 and $1.20 \times 10^{5}$ Bev for Case 2.

Hence, the minimum primary proton energy required to produce a coincidence in the meson telescope was determined to be $36.8 \mathrm{Bev}$ (i.e. just under $40 \mathrm{Bev}$ )。

This estimated proton energy agrees reasonably well with 
Dorman (1957) who states that from available evidence the muon energy at production is about one tenth of the responsible proton energy。

Assuming a differential primary proton spectrum of the form

$$
n(E) d E=0.3 E^{-2.5} \mathrm{dE} \text { for } E \geq 40 \mathrm{Bev},
$$

the average energy $\langle\mathbb{E}\rangle$ of the primary particles responsible for the counting rates encountered in this investigation was readily determined as

$$
\langle E\rangle=\frac{\int_{\Delta 0}^{\infty} \operatorname{En}(E) d E}{\int_{\Delta=0}^{\infty} n(E) d E} \div 120 \text { Bev. }
$$


APPENDIX II - CALCULATION OF ZENITH AND AZIMUTH ANGLES

It is required to calculate the zenith and azimuth orientations of the telescope as a function of local solar time. The zenith angle is defined as the angle between the vertical (with respect to the earth) and the longitudinal axis of the telescope. The azimuth angle is the angle that the projection of the telescope onto the horizontal plane (with respect to the earth) makes with the geographic North-South plane passing vertically through the polar axis.

Consider first the zenith angle $\phi$.

As the telescope rotates around the polar axis at the rate of 1 revolution per solar day either end of the telescope traces out a circular path, the plane of which is inclined to the zenith at an angle of $49.9^{\circ}$ (the geographic latitude of Winnipeg, Manitobal.

In figure $27 A B$ represents the telescope at some time during the local solar day; $z^{\prime}$ represents the direction of

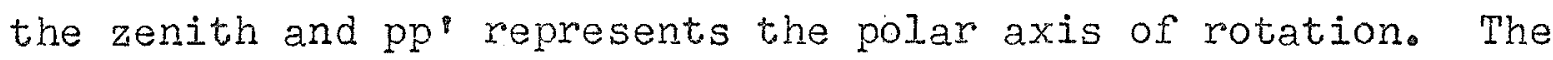
zenith angle $D$ is the angle between $A B$ and $z z^{9} \cdot \omega$ represents the angular velocity of the points $A$ and $B$ about the intersection point 0 . The circular path traced out daily by the telescope $A B$ is also shown. Introduce an angle $\theta$, which will be a function of time $t$, representing the position of the telescope $A B$ in its circular path at any particular time of day. As a reference condition, let $\theta=0$ at the time when $A B$ 


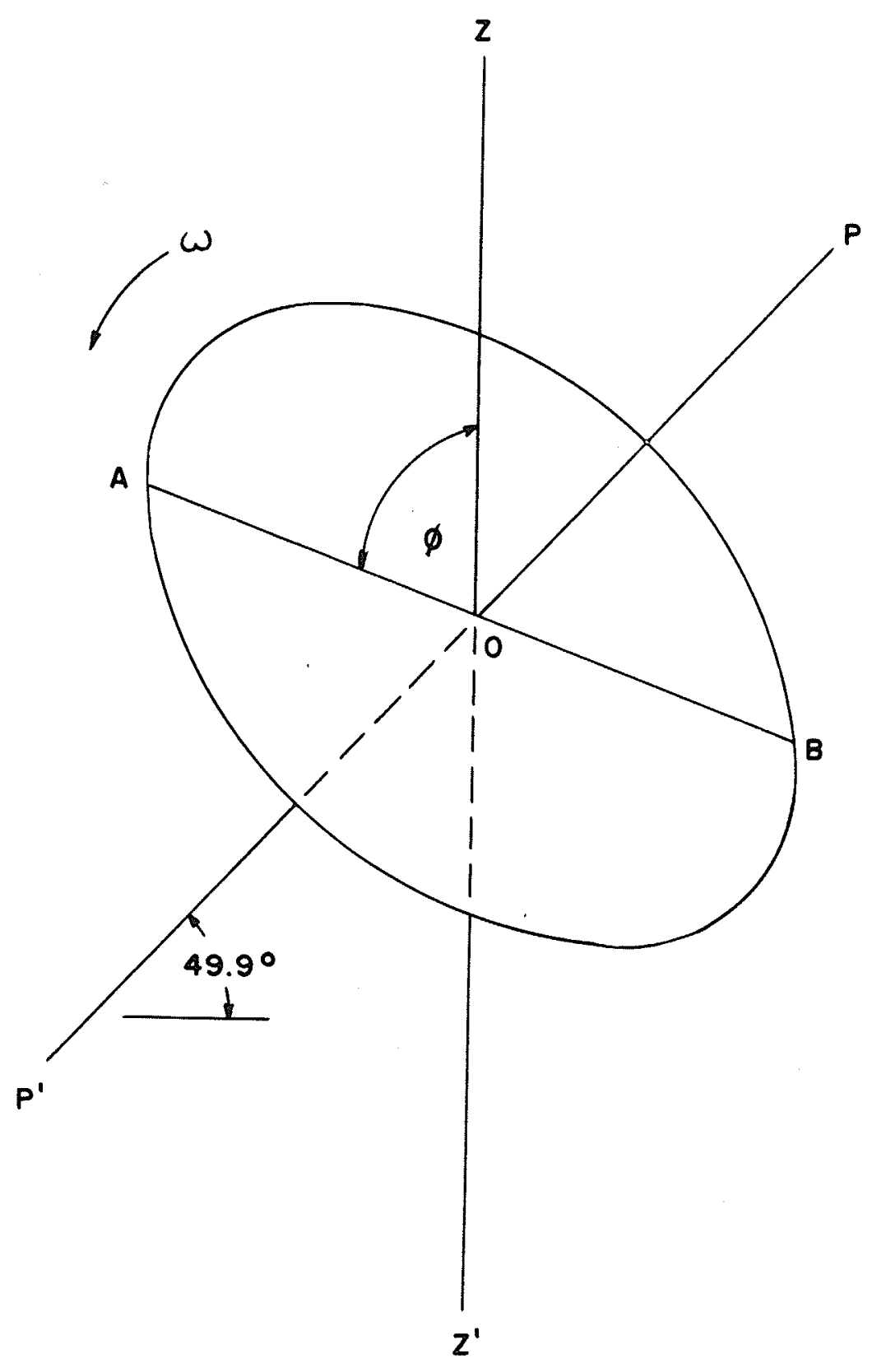


lies in the vertical plane containing the polar axis. The corresponding zenith angle $\phi$ at this time will thus be $49.9^{\circ}$. The angular velocity $\omega$ then becomes $\frac{d \theta}{d t}$ and has a magnitude of $15^{\circ}$ per solar hour.

An expression $\emptyset=f(\theta)=f(g(t))$ is now desired.

Consider the following representation of the problem. Fix the telescope position with respect to the lab (i.e. stop it in time) and consider the zenith direction as rotating around the telescope. This has, in essence, fixed in time and laboratory space the plane of the circular path that the telescope rotation defines. Then, since the angle between this plane and the zenith is $49.9^{\circ}$, this means that the zenith is now considered as rotating around the centre of the plane, generating a cone of half-angle $40.2^{\circ}$.

Figure 28 shows the salient features of this rotation. In this figure the zenith direction vector $O C$ is indicated

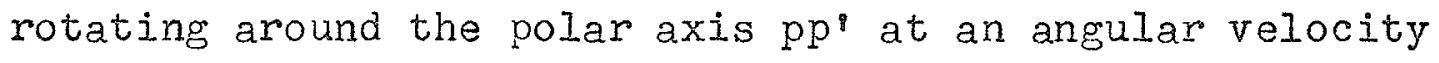
$\omega=\frac{d \theta}{d t}=15^{\circ}$ per solar hour generating the cone NOM of halfangle $40.1^{\circ}$. The fixed vector $A B$ illustrates the telescope stopped in time and laboratory space. The zenith angle $\emptyset$ is shown as the angle between $\mathrm{OC}$ and $\mathrm{OB}$.

Let $O C=\bar{C}=$ zenith direction and $O B=\bar{B}=$ fixed position of telescope. In this analysis $\bar{B}$ is constant in both magnitude and direction (its direction has been fixed in the $y$-direction). $\overline{\mathrm{C}}$ is constant in magnitude, but its direction varies with 
FIGURE 28

The Three-Dimensional Representation of a Rotating Zenith Direction 


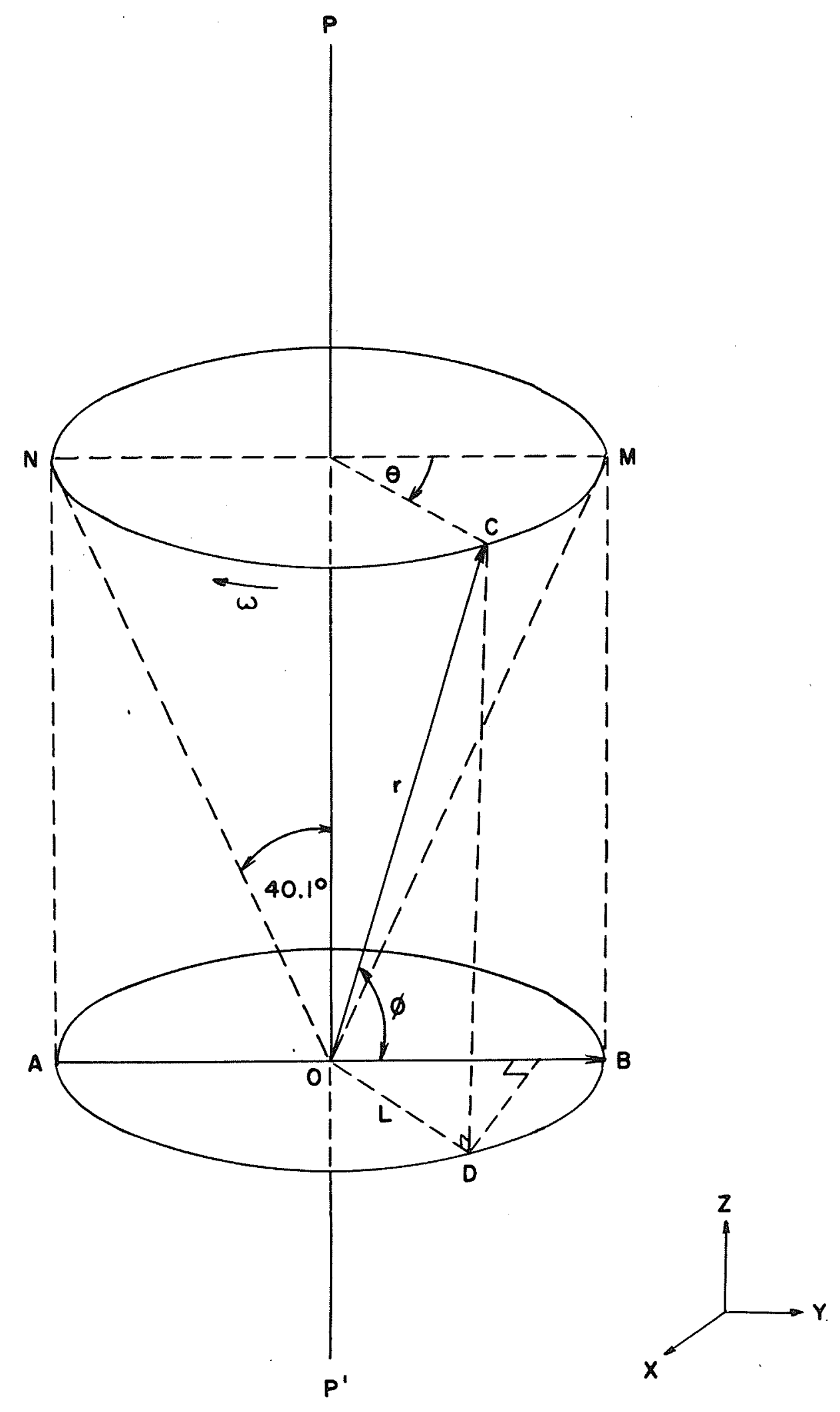




$$
\theta=f(t)
$$

Let $|\bar{C}|=r$ and $|\bar{B}|=L$. Thus $L=r \operatorname{Cos} 49.9^{\circ}$.

Consider $\bar{C} \cdot \bar{B}=\mathrm{CB} \operatorname{Cos} \phi$

$$
=r^{2} \operatorname{Cos} 49.9^{\circ} \operatorname{Cos} \phi \text {. }
$$

The scalar expansion of $\bar{C} \cdot \bar{B}$ in component form is

$$
\overline{\mathrm{C}} \cdot \overline{\mathrm{B}}=\mathrm{C}_{\mathrm{x}} \mathrm{B}_{\mathrm{x}}+\mathrm{C}_{\mathrm{y}} \mathrm{B}_{\mathrm{y}}+\mathrm{C}_{\mathrm{z}} \mathrm{B}_{\mathrm{z}} \cdot
$$

The $x, y, z$ components of $\bar{C}$ and $\bar{B}$ are readily obtained by dropping the perpendicular from $C$ to $D$ on the lower plane in figure 28.

The components of $\overline{\mathrm{C}}$ are then $\underline{i} \operatorname{Cos} 49.9^{\circ} \operatorname{Sin} \theta ; j \mathrm{j} \operatorname{Cos} 49.9^{\circ} \operatorname{Cos} \theta$ and $\underline{\operatorname{rrCos}} 40.1^{\circ}$.

The components of $\overline{\mathrm{B}}$ are $\underline{\mathrm{i}} \mathrm{O} ; \underline{\mathrm{j}} \mathrm{Cos} 49.9^{\circ}$ and $\underline{\mathrm{k} O}$.

Thus, equating the two expressions for the dot product $\bar{C} \cdot \bar{B}$, the following identity defining the zenith angle $\varnothing$ of the telescope as a function of local solar time is obtained:

$$
\operatorname{Cos} \phi=\operatorname{Cos} \theta \operatorname{Cos} 49.9^{\circ}
$$

Consider now the azimuth angle $\psi$.

The azimuth angle $\psi$ is the angle that the projection of the telescope onto the horizontal plane makes with some vertical reference plane passing through the zenith direction. This vertical reference plane is taken as the North-South plane, north and south being local geographic directions, and is the vertical plane containing the polar axis of the telescope. 
FIGURE 29

The Three-Dimensional Representation of the Azimuthal Arrangement in the Laboratory System 


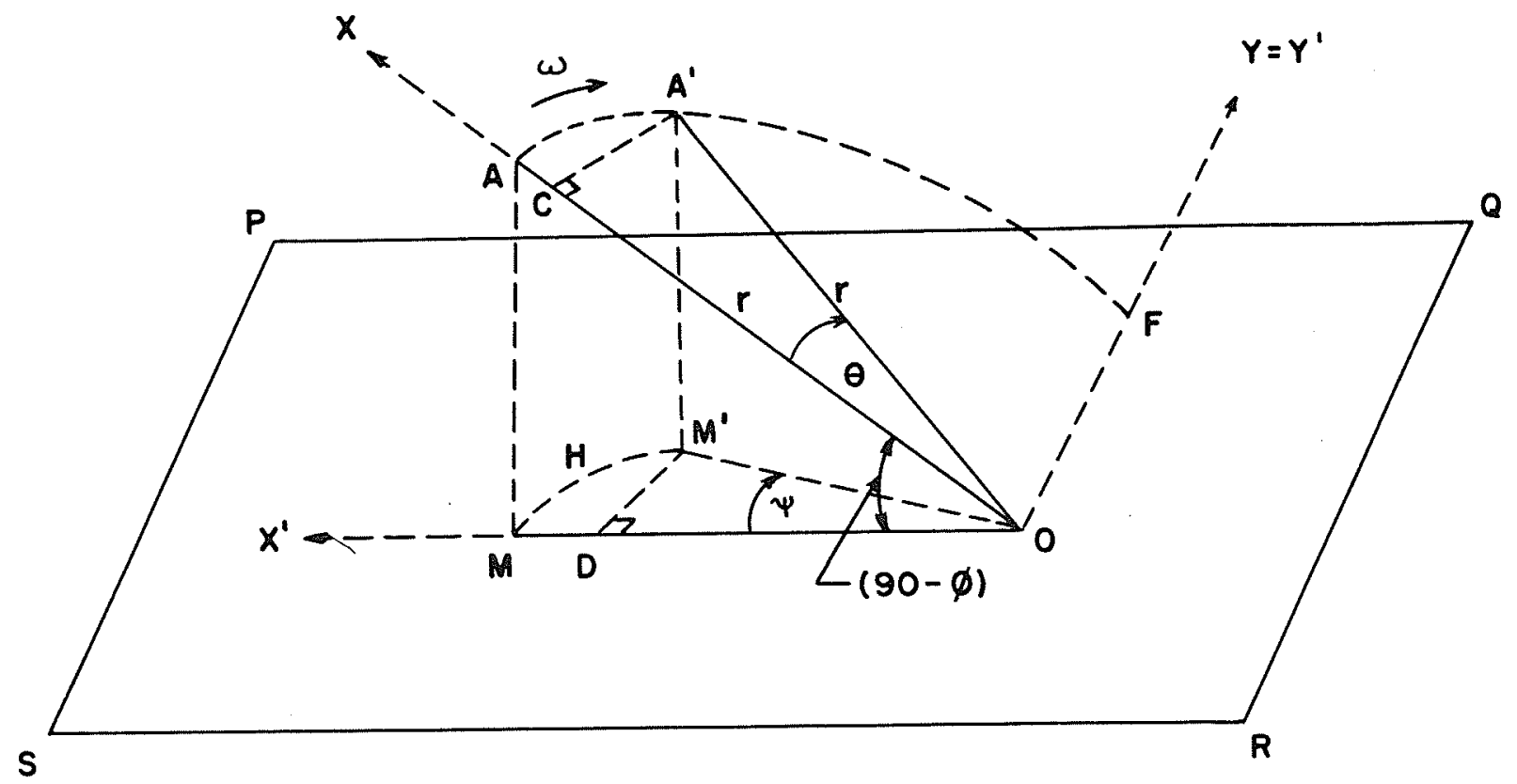


Figure 29 depicts the three-dimensional situation existing in the laboratory system. In this illustration PQRS represents a horizontal plane in the laboratory. The intersection point 0 of the telescope and the polar axis lies in this plane. The telescope (magnitude $2 \mathrm{r}$ ) is shown in an initial position OA and some later position $\mathrm{OA}^{\prime}$, this later position being defined from $\omega=\frac{d \theta}{d t}=15^{\circ} /$ solar hour. The curve $A A^{\prime} F$ is one quadrant of the circular path which the telescope traces out daily. The curve MHM represents the non-circular path traced out by the vertical projection of $A$ onto the plane PQRS. The azimuth angle $\psi$ is as shown, and the zenith angle $\emptyset$ is the angle between any position $O A$ and the perpendicular drawn to $P Q R S$ from $O$.

Two co-ordinate systems $(x, y, z)$ and $\left(x^{8}, y^{8}, z^{p}\right)$ are considered originating from the point $\mathrm{O}$. The $\mathrm{x}$-axis is taken to lie along the initial orientation $O A$ of the telescope while the $x^{\prime}$ axis is taken to lie along its projection OM in the horizontal plane. The z-axis (not shown) is taken to be perpendicular to the circular path $\mathrm{AA}^{\mathrm{P} F}$ and directed out of the paper, while the $z^{i}$-axis lies along the zenith direction (i॰e. perpendicular to the horizontal plane). The y-axis coincides with the $\mathrm{y}^{i}$-axis as indicated.

The transformation equations between the primed and the unprimed systems are readily obtained as 


$$
\begin{aligned}
& x^{p}=r \operatorname{Cos}(90-\emptyset) \operatorname{Cos} \psi \\
& y^{\prime}=r \operatorname{Sin} \theta \\
& z^{\prime}=r \operatorname{Sin}(90-\varnothing)
\end{aligned}
$$

The azimuth angle $\psi$ is given by the relation

$$
\tan \psi=\frac{y^{9}}{x^{T}}
$$

Using the transformation equations, $\psi$ is determined from the identity

$$
\sin \psi=\frac{\sin \theta}{\operatorname{Sin} \phi} \ldots \ldots \ldots(2)
$$

Substituting the expression for $\emptyset$ from equation (I) yields

$$
\sin \psi=\frac{\sin \theta}{\sqrt{1-\operatorname{Cos}^{2} \theta \operatorname{Cos}^{2} 49.9^{\circ}}}
$$

Thus, once a reference value of $\theta$ is chosen, equations (1) and (2) may be used to evaluate the zenith orientation of the telescope at any time during the solar day along with the corresponding azimuth orientation. The initial value of $\theta$ was chosen to be zero at the instant the telescope was in the vertical plane containing the polar axis. The time of day corresponding to this value of $\theta$ is, of course, totally arbitrary, as the telescope rotation could be started at any desired time with its orientation in the vertical plane. Table II-I gives the tabulated spectrum of hourly values of 
$\varnothing$ and $\psi$ computed from equations (I) and (2). The table starts with the maximum zenith angle of $49.9^{\circ}$ (i.e. with the telescope in the most vertical position). The local time for this condition is arbitrary。

\section{TABLE II-1}

TABULATED HOURLY VALUES OF ZENITH AND AZTMUTH ANGLES FOR ONE DAILY ROTATION OF THE POLAR AXIS

Zenith Angle $(\phi)$

$49.9^{\circ}$

51.5

56.1

62.9

71.2

30.4

90.0

80.4

$71 \cdot 2$

62.9

56.1

51.5

49.9

51.5

56.1

62.9

71.2

80.4

90.0

80.4

71.2

62.9

56.1

51.5

49.9
Azimuth Angle ( $\psi)$

\begin{tabular}{|c|c|c|}
\hline $0^{\circ}$ & Sout? & \\
\hline 19.3 & West & of South \\
\hline 52.6 & & $p$ \\
\hline 66.2 & & $\pi$ \\
\hline 81.8 & & $n$ \\
\hline 90.0 & West & \\
\hline $\begin{array}{l}98.2 \\
13.8\end{array}$ & West & of South \\
\hline 27.4 & & $n$ \\
\hline $\begin{array}{l}4.43 .0 \\
60.7\end{array}$ & & $n$ \\
\hline 80.0 & North & \\
\hline $\begin{array}{l}60.7 \\
4.3 .0\end{array}$ & East & of South \\
\hline 27.2 & & ๓ \\
\hline 30 & & $\pi$ \\
\hline 98.2 & & n \\
\hline 0.0 & East & \\
\hline $\begin{array}{l}81.8 \\
66.2\end{array}$ & East & of South \\
\hline 52.6 & & $\pi$ \\
\hline & & $\dddot{m}$ \\
\hline 19. & & $?$ \\
\hline & South & \\
\hline
\end{tabular}


APPENDIX III - THE ALIGNMENT OF THE POLAR AXIS

The problem of fixing the polar axis on the North Pole Star was complicated by the fact that the telescope was (and is) located inside a windowless cosmic ray laboratory situated on the roof of the Allen Physics Building. Hence, no direct method of visual alignment from the location of the detecting system could be employed. Rather, the line-up procedure utilized an engineer's transit and a method of triangulation.

The position of a star in the horizon system is denoted by its altitude and its azimuth. The azimuth of a star is the angular distance measured from the north point eastward along the horizon to the vertical circle of the star. The altitude of a star is its angular distance above the horizon. Figure $30(a)$ illustrates these two position co-ordinates on the celestial sphere for a star $S$.

It may readily be seen that the altitude of the North Star as observed at a location of geographic latitude $\lambda$ is just $\lambda$ itself. Thus the altitude of the North Star had been explicity accounted for in the equatorial mount wherein the polar axis was mounted at an angle of $49.9^{\circ}$ ( $\lambda$ for Winnipeg) to the horizontal. The problem of alignment was then reduced to the necessity of coinciding the vertical plane containing the polar axis with the azimuth circle of the Pole star. It was to this purpose that the triangulation method was employed. The laboratory contained one door which gave access to 
FIGURE 30

(a) Position Coordinates on the Celestial Sphere

(b) Triangulation Method Used to Align the Polar Axis With the North Star 
(a)

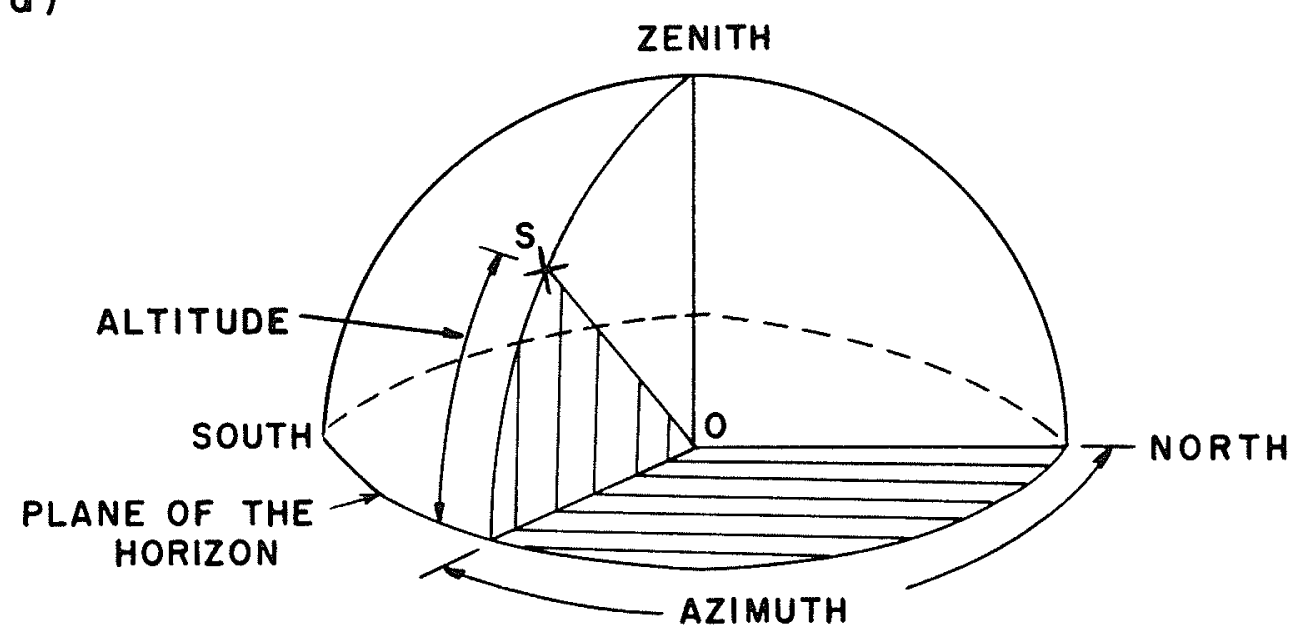

(b)

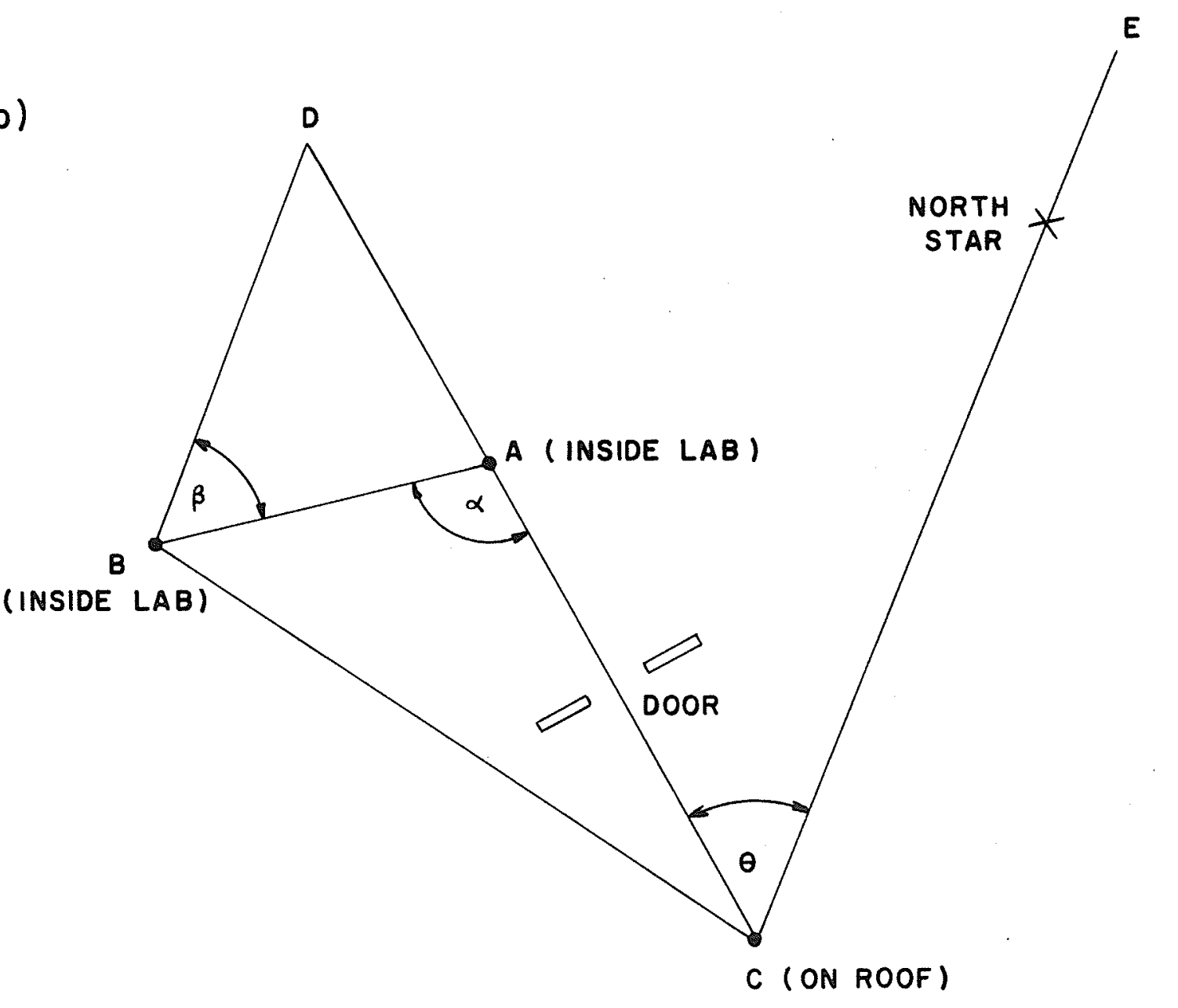


the roof of the building. Utilizing this fact, three points $A, B$ and $C$ were selected and marked, points $A$ and $B$ being on the floor of the laboratory and $C$ outside the laboratory on the roof of the building. These three points were then used as transit stations and their orientation is shown in figure 30(b). In this figure the projection of the North Star on the horizontal plane is shown and the angles $\alpha, \beta$ and $\theta$ are as indicated. The lines $\mathrm{BD}$ and $\mathrm{CE}$ are parallel.

Clearly,

$$
\beta=\alpha-\theta
$$

With the transit levelled over station A, sights on $B$ and $c$ determined the angle $\alpha$. With the transit levelled over station $C$, sights on $A$ and the North Star (the alignment was necessarily done on a clear evening when the Pole Star was distinctly visible) determined the angle $\theta_{\text {e }}$ Once $\alpha$ and $\theta$ were known, the above relation readily gave the angle $\beta$.

The astronomical mount was then brought manually into a position such that with the transit over station $B$, the left edge of the polar axis (as viewed from $B$ ) was in coincidence with the direction $B D$ (i.e. an angular distance $B$ from $A B$ ). This type of triangulation was performed on three separate evenings, each time using values of $\theta$ and $\alpha$ which were the averages of several independent readings of the transit. These independent trials were consistent to within approximately $0.028 \%$ 
In order to preserve the location of the North Star with respect to a reference point inside the laboratory, the transit was levelled at $B$ and turned exactly $180^{\circ}$ from the left edge of the polar axis (as viewed from B). Two small points were then located on the laboratory wall in line with the left edge of the axis and station B. Use was made of these points periodically throughout the course of the research to ensure the consistency with time of the polar axis alignment. 


\section{REFERENCES}

Ahluwalia, H.S. and Dessler, A.J. (1962), Planet, Space Sci。, 2, 195

Alfven, Ho (1949), Phys, Revo, 75, 11, 1732

Alfven, H. (1954), Tellus, 6, 232

Alfven, H. (1958), Nuovo Cimento (10), 定, Suppl. 2, 491

Anderson, K.A. (1960), Scientific American, 64, June 1960

Auger, P. (1936), Journal de Physique et le Radium, I, 58

Barret, $\mathrm{P}_{0} \mathrm{H}_{0}$, Bollinger, L。 $\mathrm{M}_{0}$, Cocconi, $G_{0}$, Eisenberg, $\mathrm{Y}_{0}$ and Griesen, K。 (1952), Rev。 Mod. Phys., 24, 133

Bercovitch, $N_{0}$ (1962), Appendix II, Chalk River Letter, File No. 212-2-10, Dec. 1962

Biermann, Lo (1957), Observatory, 107, 109

Blackett, P.MoS. (1938), Phys. Revo, 54, 973

Brunberg, E. (1953), J. Geophys。 Res., 58, 272

Brunberg, E. and Dattner, A. (1954), Tellus, 6, 73

Budini, P. and Moliere, G. (1953), Kosmiche Strahlung, ed. W.

Bukata, R.P. (1960), Master's Thesis, University of Manitoba Bukata, R.P., Chin, F。K. and Standil, S。 (1962), Can。 J. Phys。,

Chin, F.K. Bukata, R.P. and Standil, S* (1962), Can॰ Jo Phys.,

Clark, Go, Earl, Jo, Kraushaar, Wo, Linsley, Jo, Rossi, B. and Scherb, F。 (1957), Nature, 180, 406

Clark, Go, Earl, Jo, Kraushaar, Wo, Linsley, Jo, Rossi, B. , Scherb, Fo and Scott, D。 (1961), Phys. Rev, 122, 637

Cocconi, G. (1961), Handbuch der Physik, Vol XLVI/I Cosmic Rays 1,263 
Cogger, L。L. (1960), Atomic Energy of Canada Ltd. Report $A E C L-1104$ (not published)

Conversi, Mo (1950), Phys, Revo, 79, 749

Coxell, Ho, Fairchild, W.W. and Pomerantz, M.A. (1963), Report from Bartol Research Foundation of the Franklin Institute (not published)

Dorman, L。I. (1957), Cosmic Ray Variations, State Publishing House, Moscow (Translation Tech. Doc。 Liaison Office, Wright-Patterson Air Force Base, Wa shington, 1958)

Dorman, L. I. (1963), Progress in Elementary Particle and Cosmic Ray Physics, Vol VII, Ch. 4

Duperier, A. (1944), Terr, Magn,Atmos, Elect, 4 49,1

Dwight, K。 (1950), Phys。 Rev。, 78, 40

Ehmert, A。 (I959), Proc。 I.U॰ P.A.P. Cosmic Ray Conf॰, Moscow, 4. 142

Elliot, H. (1952), Progress in Cosmic Ray Physics, Vol I, 455

Elliot, Ho (1957), Annals of the IGY, VII, 374

Elliot, Ho (1960), Phil, Mage, 5, 54, 601

Fenton, A.G., Fenton K.B. and Rose, D.C. (I958), Can.J. Phys., 36, 824

Fenton, A.G॰, Jacklyn, R。M. and Taylor, R. B. (196I), Nuovo

Cimento, $\underline{22}, 285$

Fermi, E. (1949), Phys, Revo, 75, 1169

Finch, H.P。 and Leaton, B.R. (1957), Monthly Notices Roy. Astron。 Soc. Geophys. SuppI. I, 314

Firor, Jo (1954), Phys, Revo, 94, 1017

Forbush, S.E. (1954), J. Geophys。 Res., 59, 525

Fowler, G.No and Wolfendale, A.W. (1961), Handbuch der Physik, Vol XLVI/I Cosmic Rays 1,301

Galbraith, W. (1958), Extensive Air Showers, London Butterworths 
Gammel, J.L. and Thaler, R.M. (1960), Progress in Elementary Particle and Cosmic Ray

George, E。 Po (1952), Progress in Cosmic Ray Physics, Vol I, Glaser, D.A., Hamermesh, Bo and Safonov, G。 (1950), Phys。 Rev。, 80, 625

Griesen, Ko (1956), Progress in Cosmic Ray Physics, Vol I, I Hasegawa, Ho, Matano, T., Miura, Io, Oda, Mo, Tanahashi, Go, Tanaka, Y. Higashi, S. , Kitamura, T., Mishima, $Y_{\bullet}$, Miyamoto, S., Shibata, Ko and Watese, $Y_{0}(1962)$,

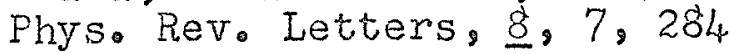

Hatton, C.J. and Carswell, D.A. (1963), Atomic Energy of Canada Ltd. , Report AECL-I824 (unpublished)

Hess, V.F. (1942), Rev。 Mod, Physo, 11, 153

Hogg, A.R。 (1947), Proc, Roy, Soc。, AI92, 128

IG Bulletin (1964), Initial Results from the First Interplanetary Monitoring Platform (IMP-I), N.A.S., IG Bulletin, 84, June 1964

Jacklyn, R。M. (1962), Nuovo Cimento, 214, 1034

Jacklyn, R.M. (1963), Nuovo Cimento, 30, 40

Janossy, Lo and Rochester, G.D. (1944), Royal Soc, of London, A182, 183, 186

Katzman, J. (1959), Can. J. Phys, 37, 1207

Kraushaar, W.L. (1949), Phys. Reve, 76, 1045

Lapointe, S.M. and Rose, D.C. (1962), $\begin{gathered}\text { Can。 J. Phys, } \\ 687\end{gathered}$

Leighton, R.B. (1959), Principles of Modern Physics, Appendix F,

Linsley, Jo, Scarsi, Lo, Eccles, P.J. and Rossi, B. (1962), Phys. Rev. Letters, 安, 7, 286

Malmfors, K.F。(1945), Arkiv Mat, Astron. Fysik, A32, No. 8 
Mathews, T. (1962), Ph.D Thesis, Imperial College, London McCracken, KoG. (1962), J. Geophys, Reso, 67, 2, 423 Moroney, J.R. and Parry, J.K. (1954), Austral. J. Phys., I, 423 Morrison, P. (1956), Phys, Rev。, 101, 1397 Morrison, Po (196I), Handbuch der Physik, Vol XLVI/I Cosmic Rays Myssowsky, Lo and Tuwim, Lo $\left(192 \delta^{\circ}\right)$, Z. Phys, , 50, 273 Nagashima, K. (1951), J. Geomag. Geoelec., 3, 100 Owen, B.G. and Wilson, J.G. (1955), Proc. Phys. Soc., A68, 409 Parker, E.N. (1958), Astrophys. Jo, 128, 664 Parker, E.No (1963), Interplanetary Dynamical Processes

Perkins, DoH. (1961), Cern International Conference on Theoretical. Aspects of Very High Energy Phenomena, Geneva, Aug. 11, 1961

Peters, B. (1952), Progress in Cosmic Ray Physics, Vol I, 191

Peters, B. (I959), J. Geophys, Rese, 64, 2, 155

Puppi, $G_{\circ}$ and Dallaporta, $N_{*}(1952), \frac{\text { Progress in Cosmic Ray }}{\text { Physics, Vol 1, Ch. } \sigma_{0}}$

Puppi, Go (1956), Progress in Cosmic Ray Physics, Vol III, 345 Quenby, J.J. and Webber, W.R. (1959), Phil. Nag., 4, 90 Rao, U。Ro, McCracken, $K_{0} G_{0}$ and Venkatesan, Do (1963), Jo Geophys。 Res., 68, 345

Ray, E.C. (196I), Handbuch der Physik, Vol XLVI/I, Cosmic Rays I, 130

Richtmeyer, R.D. and Teller, E. (1949), Phys, Reve, 75, II, 1729 Roe, B.Po and Ozaki, So (1959), Bult, Am, Phys, Soc, , 4,8 Rossi, B. (1933), Z Physik, sz2, 15I 
Rossi, Bo and Griesen, Ko (1941), Rev。 IJod. Phys, 13, 240 Rossi, B. (1948), Rev, lod. Phys, 20, 537 Rossi, B. (1952), High Energy Particles, Prentice-Hall, New York Rossi, B. (1959), Scientific American, 201, 135 (November) Rothwell, P. (1959), J. Geophys, Res., 64, 2026

Sakakibara, So and Sekido, Y. (1964), Phys, Revo, 133, 2B, 529 Schluter, A. (195l), Z Naturforsch, 6a, 613 Simpson, J.A., Fonger, W。 and Treiman, S.B. (1952), Phys。 Rev。, 90, 5,934

Simpson, J.A. (1956), Annals of the IGY, 349

Simpson, J.A. (1957), Annals of the IGY, VII, 351

Simpson, J.A. (1958), Electromagnetic Phenomena in Cosmical Physics, Cambridge University Press,

Singer, S.F。 (1958a), Progress in Elementary Particle and Cosmic Ray Physics, Vol IV, 245

Singer, S.F. (1958b), Nuovo Cimento, $\underline{8}$, Suppl II, 334

Sitte, K。 (1961), Handbuch der Physik, Vol XIVI/I Cosmic Rays I, Smith, J.A. and Duller, N.M. (1959), J. Geophys, Res., 64, 2297 Standil, So and Bukata, R.P. (196/4a), Anisotropy of Primary Cosmic Radiation at the Earth, paper presented at the C.A.P. meeting in Halifax, N.S., June $11-13$

Standil, So and Bukata, R.P. (1964b), Evidence for Galactic Origin of Observed Anisotropy of Cosmic Radiation at the Earth, post-deadline paper presented at the A.P.S. summer meeting in Denver, Col., June 25-27

Standil, So and Bukata, R。P. (1964c), Phys, Rev。 Letters, 12, 17,487 
Stormer, C。 (1931), Z Astrophys., 3, 31

Stormer, C. (1955), The Polar Aurora, Oxford University Press

Thorndike, A。M (1952), $\frac{\text { Mesons: A Summary of Experimental Facts, }}{\text { McGraw-Hill Co, Inco New York }}$

Trefall, H. (1955), Proc. Phys. Soc. of London, A68, 953

Wada, Mo (1960), Scientific Papers of the Institute of Physical and Chemical Research, 54, 4, 335

Wada, M. (1964), Private Communication from M.I.T., Boston

Wadàington, C.J. (1960), Prog. Nucl。Phys., 音, I

Webber, W.R. (1962), $\frac{\text { Progress in Elementary Particle and Cosmic }}{\text { Ray Physics, Vol VI, } 224}$ 